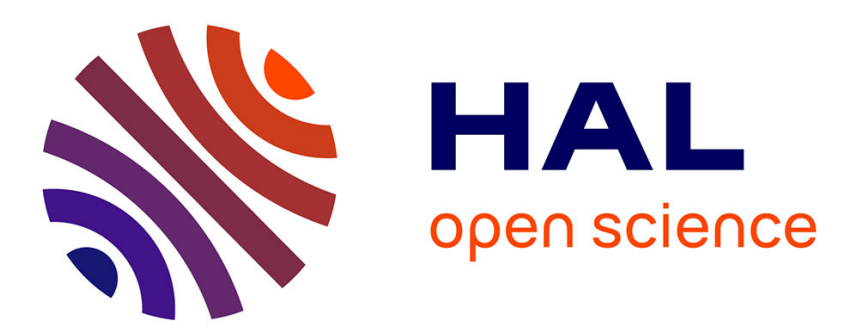

\title{
Exponential boundary feedback stabilization of a shock steady state for the inviscid Burgers equation
}

Georges Bastin, Jean-Michel Coron, Amaury Hayat, Peipei Shang

\section{To cite this version:}

Georges Bastin, Jean-Michel Coron, Amaury Hayat, Peipei Shang. Exponential boundary feedback stabilization of a shock steady state for the inviscid Burgers equation. Mathematical Models and Methods in Applied Sciences, 2019, 29 (2), pp.271-316. 10.1142/S021820251950009X . hal-01723361v2

\section{HAL Id: hal-01723361 \\ https://hal.science/hal-01723361v2}

Submitted on 1 Feb 2019

HAL is a multi-disciplinary open access archive for the deposit and dissemination of scientific research documents, whether they are published or not. The documents may come from teaching and research institutions in France or abroad, or from public or private research centers.
L'archive ouverte pluridisciplinaire HAL, est destinée au dépôt et à la diffusion de documents scientifiques de niveau recherche, publiés ou non, émanant des établissements d'enseignement et de recherche français ou étrangers, des laboratoires publics ou privés. 


\title{
Exponential boundary feedback stabilization of a shock steady state for the inviscid Burgers equation
}

\author{
Georges Bastin ${ }^{\mathrm{a}}$, Jean-Michel Coron ${ }^{\mathrm{b}, \mathrm{d}}$, Amaury Hayat ${ }^{\mathrm{b}, \mathrm{e}}$, and Peipei Shang ${ }^{\mathrm{b}, \mathrm{c}, \mathrm{e}}$ \\ ${ }^{a}$ Department of Mathematical Engineering, ICTEAM, University of Louvain, Louvain-La-Neuve, Belgium. \\ ${ }^{\mathrm{b}}$ Laboratoire Jacques-Louis Lions, Université Pierre et Marie Curie, Paris, France. \\ ${ }^{\mathrm{c}}$ School of Mathematical Sciences, Tongji University, Shanghai, China. \\ ${ }^{\mathrm{d}}$ Institute for Theoretical Studies, ETH, Zurich, Switzerland. \\ ${ }^{\mathrm{e}}$ Forschungsinstitut für Mathematik (FIM), ETH, Zurich, Switzerland.
}

\begin{abstract}
In this paper, we study the exponential stabilization of a shock steady state for the inviscid Burgers equation on a bounded interval. Our analysis relies on the construction of an explicit strict control Lyapunov function. We prove that by appropriately choosing the feedback boundary conditions, we can stabilize the state as well as the shock location to the desired steady state in $H^{2}$-norm, with an arbitrary decay rate.
\end{abstract}

\section{Introduction}

The problem of asymptotic stabilization for hyperbolic systems using boundary feedback control has been studied for a long time. We refer to the pioneer work due to Rauch and Taylor 38 and Russell 39 . for linear coupled hyperbolic systems. The first important result of asymptotic stability concerning quasilinear hyperbolic equations was obtained by Slemrod [41] and Greenberg and Li [19. These two works dealt with local dissipative boundary conditions. The result was established by using the method of characteristics, which allows to estimate the related bounds along the characteristic curves in the framework of $C^{1}$ solutions. Another approach to analyze the dissipative boundary conditions is based on the use of Lyapunov functions. Especially, Coron, Bastin and Andrea-Novel [13] used this method to study the asymptotic behavior of the nonlinear hyperbolic equations in the framework of $H^{2}$ solutions. In particular, the Lyapunov function they constructed is an extension of the entropy and can be made strictly negative definite by properly choosing the boundary conditions. This method has been later on widely used for hyperbolic conservation laws in the framework of $C^{1}$ solutions [11, 20, 21 . or $H^{2}$ solutions [2, 4, 5, 10, 12, 17, 22] (see [3] for an overview of this method).

But all of these results concerning the asymptotic stability of nonlinear hyperbolic equations focus on the convergence to regular solutions, i.e., on the stabilization of regular solutions to a desired regular steady state. It is well known, however, that for quasilinear hyperbolic partial differential equations, solutions may break down in finite time when their first derivatives break up even if the initial condition is smooth 29 . They give rise to the phenomena of shock waves with numerous important applications in physics and fluid mechanics. Compared to classical case, very few results exist on the stabilization of less regular solutions, which requires new techniques. This is also true for related fields, as the optimal control problem [9, 37. For the problem of control and asymptotic stabilization of less regular solutions, we refer to [7] for the controllability of a general hyperbolic system of conservation laws, 6, 35] for the stabilization in the scalar case and [7, 14] for the stabilization of a hyperbolic system of conservation laws. In [6, 14, 35, by using suitable feedback laws on both side of the interval, one can steer asymptotically any initial data with sufficiently small total variations to any close constant steady states. All those results concern the boundary stabilization of constant steady states. In particular, as the target state is regular there is no need to stabilize any shock location. In this work, we will study the boundary stabilization of steady states with jump discontinuities for a scalar equation. We believe that our method can be applied to nonlinear hyperbolic systems as well. While preparing the revised version, our attention was drawn to a very recent work [36] studying a 
similar problem in the BV norm. The method and the results are quite different and complementary to this work.

Hyperbolic systems have a wide application in fluid dynamics, and hydraulic jump is one of the best known examples of shock waves as it is frequently observed in open channel flow such as rivers and spillways. Other physical examples of shock waves can be found in road traffic or in gas transportation, with the water hammer phenomenon. In the literature, Burgers equation often appears as a simplification of the dynamical model of flows, as well as the most studied scalar model for transportation. Burgers turbulence has been investigated both analytically and numerically by many authors either as a preliminary approach to turbulence prior to an occurrence of the Navier-Stokes turbulence or for its own sake since the Burgers equation describes the formation and decay of weak shock waves in a compressible fluid [26, 32, 44. From a mathematical point of view, it turns out that the study of Burgers equation leads to many of the ideas that arise in the field of nonlinear hyperbolic equations. It is therefore a natural first step to develop methods for the control of this equation. For the boundary stabilization problem of viscous Burgers equation, we refer to works by Krstic et al. [28, 42 for the stabilization of regular shock-like profile steady states and [8, 27] for the stabilization of null-steady-state. In 42, the authors proved that the shock-like profile steady states of the linearized unit viscous Burgers equation is exponentially stable when using high-gain "radiation" boundary feedback (i.e. static boundary feedback only depending on output measurements). However, they showed that there is a limitation in the decay rate achievable by radiation feedback, i.e., the decay rate goes to zero exponentially as the shock becomes sharper. Thus, they have to use another strategy (namely backstepping method) to achieve arbitrarily fast local convergence to arbitrarily sharp shock profiles. However, this strategy requires a kind of full-state feedback control, rather than measuring only the boundary data.

In this paper, we study the exponential asymptotic stability of a shock steady state of the Burgers equation in $\mathrm{H}^{2}$-norm, which has been commonly used as a proper norm for studying the stability of hyperbolic systems (see e.g. [16, 24, 43]), as it enables to deal with Lyapunov functions that are integrals on the domain of quadratic quantities, which is relatively easy to handle. To that end, we construct an explicit Lyapunov function with a strict negative definite time derivative by properly choosing the boundary conditions. Though it has been shown in 15 that exponential stability in $H^{2}$-norm is not equivalent to $C^{1}$-norm, our result could probably be generalized to the $C^{1}$-norm for conservation laws by transforming the Lyapunov functions as in [11, 20.

The first problem is to deal with the well-posedness of the corresponding initial boundary value problem (IBVP) on a bounded domain. The existence of the weak solution to the initial value problem (IVP) of Burgers equation was first studied by Hopf by using vanishing viscosity [23. The uniqueness of the entropy solution was then studied by Oleinik [34. One can refer to 29 for a comprehensive study of the well-posedness of hyperbolic conservation laws in piecewise continuous entropy solution case and also to [18] in the class of entropy BV functions. Although there are many results for the well-posedness of the (IVP) for hyperbolic conservation laws, the problem of (IBVP) is less studied due to the difficulty of handling the boundary condition. In [1, the authors studied (IBVP) but in the quarter plane, i.e., $x>0, t>0$. By requiring that the boundary condition at $x=0$ is satisfied in a weak sense, they can apply the method introduced by LeFloch [30] and obtain the explicit formula of the solution. However, our case is more complicated since we consider the Burgers equation defined on a bounded interval.

The organization of the paper is the following. In Section 2, we formulate the problem and state our main results. In Section 3 , we prove the well-posedness of the Burgers equation in the framework of piecewise continuously differentiable entropy solutions, which is one of the main results in this paper. Based on this well-posedness result, we then prove in Section 4 by a Lyapunov approach that for appropriately chosen boundary conditions, we can achieve the exponential stability in $\mathrm{H}^{2}$-norm of a shock steady state with any given arbitrary decay rate and with an exact exponential stabilization of the desired shock location. This result also holds for the $H^{k}$-norm for any $k \geqslant 2$. In Section 5 , we extend the result to a more general convex flux by requiring some additional conditions on the flux. Conclusion and some open problems are provided in Section 6. Finally, some technical proof are given in the Appendix. 


\section{Problem statement and main result}

We consider the following nonlinear inviscid Burgers equation on a bounded domain

$$
y_{t}(t, x)+\left(\frac{y^{2}}{2}\right)_{x}(t, x)=0
$$

with initial condition

$$
y(0, x)=y_{0}(x), \quad x \in(0, L),
$$

where $L>0$ and boundary controls

$$
y\left(t, 0^{+}\right)=u_{0}(t), \quad y\left(t, L^{-}\right)=u_{L}(t) .
$$

In this article, we will be exclusively concerned with the case where the controls $u_{0}(t)>0, u_{L}(t)<0$ have opposite signs and the state $y(t,$.$) at each time t$ has a jump discontinuity as illustrated in Figure 1. The discontinuity is a shock wave that occurs at position $x_{s}(t) \in(0, L)$. According to the

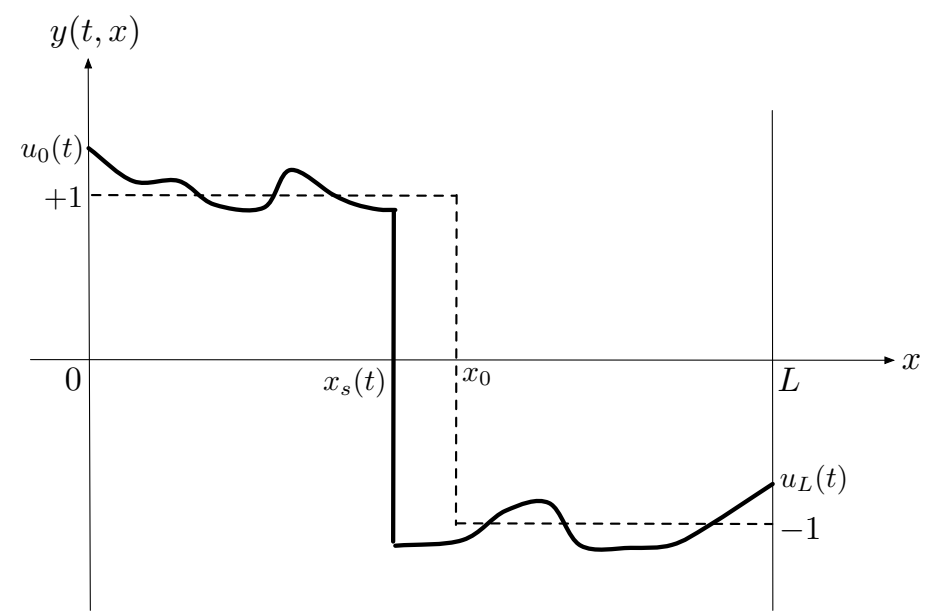

Figure 1: Entropy solution to the Burgers equation with a shock wave.

Rankine-Hugoniot condition, the shock wave moves with the speed

$$
\dot{x}_{s}(t)=\frac{y\left(t, x_{s}(t)^{+}\right)+y\left(t, x_{s}(t)^{-}\right)}{2}
$$

which satisfies the Lax entropy condition 29

$$
y\left(t, x_{s}(t)^{+}\right)<\dot{x}_{s}(t)<y\left(t, x_{s}(t)^{-}\right),
$$

together with the initial condition

$$
x_{s}(0)=x_{s 0} .
$$

Under a constant control $u_{0}(t)=-u_{L}(t)=1$ for all $t$, for any $x_{0} \in(0, L)$, the system (1), (3), (4) has a steady state $\left(y^{*}, x_{s}^{*}\right)$ defined as follows:

$$
\begin{aligned}
& y^{*}(x)= \begin{cases}1, & x \in\left[0, x_{0}\right), \\
-1, & x \in\left(x_{0}, L\right],\end{cases} \\
& x_{s}^{*}=x_{0} .
\end{aligned}
$$

These equilibria are clearly not isolated and, consequently, not asymptotically stable. Indeed, one can see that for any given equilibrium $y^{*}$ satisfying (7), we can find initial data arbitrarily close to $y^{*}$ which 
is also an equilibrium of the form (7). As the solution cannot be approaching the given equilibrium when $t$ tends to infinity as long as the initial data is another equilibrium, this feature prevents any stability no matter how close the initial data is around $y^{*}$. With such open-loop constant control another problem could appear: any small mistake on the boundary control could result in a nonstationary shock moving far away from $x_{0}$. It is therefore relevant to study the boundary feedback stabilization of the control system (1), (3), (4).

In this paper, our main contribution is precisely to show how we can exponentially stabilize any of the steady states defined by (7) with boundary feedback controls of the following form:

$$
\begin{aligned}
& u_{0}(t)=k_{1} y\left(t, x_{s}(t)^{-}\right)+\left(1-k_{1}\right)+b_{1}\left(x_{0}-x_{s}(t)\right), \\
& u_{L}(t)=k_{2} y\left(t, x_{s}(t)^{+}\right)-\left(1-k_{2}\right)+b_{2}\left(x_{0}-x_{s}(t)\right) .
\end{aligned}
$$

Here, it is important to emphasize that, with these controls, we are able not only to guarantee the exponential convergence of the solution $y(t, x)$ to the steady state $y^{*}$ but also to exponentially stabilize the location of the shock discontinuity at the exact desired position $x_{0}$. In practice, if the system was used for instance to model gas transportation, the measures of the state around the shock could be obtained using sensors in the pipe. Note that if the control is applied properly, sensors would be only needed on a small region as the shock would remain located in a small region.

Before addressing the exponential stability issue, we first show that there exists a unique piecewise continuously differentiable entropy solution with $x_{s}(t)$ as its single shock for system (1)-(4), (6), (8) provided that $y_{0}$ and $x_{s 0}$ are in a small neighborhood of $y^{*}$ and $x_{0}$ respectively.

For any given initial condition (2) and (6), we define the following zero order compatibility conditions

$$
\begin{aligned}
& y_{0}\left(0^{+}\right)=k_{1} y_{0}\left(x_{s 0}^{-}\right)+\left(1-k_{1}\right)+b_{1}\left(x_{0}-x_{s 0}\right), \\
& y_{0}\left(L^{-}\right)=k_{2} y_{0}\left(x_{s 0}^{+}\right)-\left(1-k_{2}\right)+b_{2}\left(x_{0}-x_{s 0}\right) .
\end{aligned}
$$

Differentiating (9) with respect to time $t$ and using (4), we get the following first order compatibility conditions

$$
\begin{gathered}
y_{0}\left(0^{+}\right) y_{0 x}\left(0^{+}\right)=k_{1} y_{0}\left(x_{s 0}^{-}\right) y_{0 x}\left(x_{s 0}^{-}\right)-k_{1} y_{0 x}\left(x_{s 0}^{-}\right) \frac{y_{0}\left(x_{s 0}^{-}\right)+y_{0}\left(x_{s 0}^{+}\right)}{2}+b_{1} \frac{y_{0}\left(x_{s 0}^{-}\right)+y_{0}\left(x_{s 0}^{+}\right)}{2}, \\
y_{0}\left(L^{-}\right) y_{0 x}\left(L^{-}\right)=k_{2} y_{0}\left(x_{s 0}^{+}\right) y_{0 x}\left(x_{s 0}^{+}\right)-k_{2} y_{0 x}\left(x_{s 0}^{-}\right) \frac{y_{0}\left(x_{s 0}^{-}\right)+y_{0}\left(x_{s 0}^{+}\right)}{2}+b_{2} \frac{y_{0}\left(x_{s 0}^{-}\right)+y_{0}\left(x_{s 0}^{+}\right)}{2} .
\end{gathered}
$$

The first result of this paper deals with the well-posedness of system (1)- (4), (6), (8) and is stated in the following theorem.

Theorem 2.1. For all $T>0$, there exists $\delta(T)>0$ such that, for every $x_{s 0} \in(0, L)$ and $y_{0} \in$ $H^{2}\left(\left(0, x_{s 0}\right) ; \mathbb{R}\right) \cap H^{2}\left(\left(x_{s 0}, L\right) ; \mathbb{R}\right)$ satisfying the compatibility conditions $\left.(9)-10\right)$ and

$$
\begin{aligned}
& \left|y_{0}-1\right|_{H^{2}\left(\left(0, x_{s 0}\right) ; \mathbb{R}\right)}+\left|y_{0}+1\right|_{H^{2}\left(\left(x_{s 0}, L\right) ; \mathbb{R}\right)} \leqslant \delta(T), \\
& \left|x_{s 0}-x_{0}\right| \leqslant \delta(T),
\end{aligned}
$$

the system (1)-(4), (6), (8) has a unique piecewise continuously differentiable entropy solution $y \in$ $\left.C^{0}\left([0, T] ; H^{2}\left(\left(0, x_{s}(t)\right) ; \mathbb{R}\right)\right) \cap H^{2}\left(\left(x_{s}(t), L\right) ; \mathbb{R}\right)\right)$ with $x_{s} \in C^{1}([0, T] ;(0, L))$ as its single shock. Moreover, there exists $C(T)$ such that the following estimate holds for all $t \in[0, T]$

$$
\begin{aligned}
|y(t, \cdot)-1|_{H^{2}\left(\left(0, x_{s}(t)\right) ; \mathbb{R}\right)}+|y(t, \cdot)+1|_{H^{2}\left(\left(x_{s}(t), L\right) ; \mathbb{R}\right)}+\left|x_{s}(t)-x_{0}\right| & \\
& \leqslant C(T)\left(\left|y_{0}-1\right|_{H^{2}\left(\left(0, x_{s 0}\right) ; \mathbb{R}\right)}+\left|y_{0}+1\right|_{H^{2}\left(\left(x_{s 0}, L\right) ; \mathbb{R}\right)}+\left|x_{s 0}-x_{0}\right|\right) .
\end{aligned}
$$

The proof of this result is given in Section 3

Our next result deals with the exponential stability of the steady state (7) for the $H^{2}$-norm according to the following definition. 
Definition 2.1. The steady state $\left(y^{*}, x_{0}\right) \in\left(H^{2}\left(\left(0, x_{0}\right) ; \mathbb{R}\right) \cap H^{2}\left(\left(x_{0}, L\right) ; \mathbb{R}\right)\right) \times(0, L)$ of the system (1), (3), (4), (8) is exponentially stable for the $H^{2}$-norm with decay rate $\gamma$, if there exists $\delta^{*}>0$ and $C>0$ such that for any $y_{0} \in H^{2}\left(\left(0, x_{s 0}\right) ; \mathbb{R}\right) \cap H^{2}\left(\left(x_{s 0}, L\right) ; \mathbb{R}\right)$ and $x_{s 0} \in(0, L)$ satisfying

$$
\begin{aligned}
& \left|y_{0}-y_{1}^{*}(0, \cdot)\right|_{H^{2}\left(\left(0, x_{s 0}\right) ; \mathbb{R}\right)}+\left|y_{0}-y_{2}^{*}(0, \cdot)\right|_{H^{2}\left(\left(x_{s 0}, L\right) ; \mathbb{R}\right)} \leqslant \delta^{*}, \\
& \left|x_{s 0}-x_{0}\right| \leqslant \delta^{*}
\end{aligned}
$$

and the compatibility conditions $(9)-(10)$, and for any $T>0$ the system (1)-(4), (6), (8) has a unique solution $\left(y, x_{s}\right) \in C^{0}\left([0, T] ; H^{2}\left(\left(0, x_{s}(t)\right) ; \mathbb{R}\right) \cap H^{2}\left(\left(x_{s}(t), L\right) ; \mathbb{R}\right)\right) \times C^{1}([0, T] ; \mathbb{R})$ and

$$
\begin{aligned}
& \left|y(t, \cdot)-y_{1}^{*}(t, \cdot)\right|_{H^{2}\left(\left(0, x_{s}(t)\right) ; \mathbb{R}\right)}+\left|y(t, \cdot)-y_{2}^{*}(t, \cdot)\right|_{H^{2}\left(\left(x_{s}(t), L\right) ; \mathbb{R}\right)}+\left|x_{s}(t)-x_{0}\right| \\
& \quad \leqslant C e^{-\gamma t}\left(\left|y_{0}-y_{1}^{*}(0, \cdot)\right|_{H^{2}\left(\left(0, x_{s 0}\right) ; \mathbb{R}\right)}+\left|y_{0}-y_{2}^{*}(0, \cdot)\right|_{H^{2}\left(\left(x_{s 0}, L\right) ; \mathbb{R}\right)}+\left|x_{s 0}-x_{0}\right|\right), \quad \forall t \in[0, T) .
\end{aligned}
$$

In (13) and (14),

$$
\begin{aligned}
& y_{1}^{*}(t, x)=y^{*}\left(x \frac{x_{0}}{x_{s}(t)}\right), \\
& y_{2}^{*}(t, x)=y^{*}\left(\frac{(x-L) x_{0}}{x_{s}(t)-L}\right) .
\end{aligned}
$$

Remark 1. At first glance it could seem peculiar to define $y_{1}^{*}$ and $y_{2}^{*}$ and to compare $y(t, \cdot)$ with these functions. However the steady state $y^{*}$ is piecewise $H^{2}$ with discontinuity at $x_{0}$, while the solution $y(t, x)$ is piecewise $H^{2}$ with discontinuity at the shock $x_{s}(t)$, which may be moving around $x_{0}$. Thus, to compare the solution $y$ with the steady state $y^{*}$ on the same space interval, it is necessary to define such functions $y_{1}^{*}$ and $y_{2}^{*}$.

Remark 2. We emphasize here that the "exponential stability for the $H^{2}{ }^{-n o r m " ~ i s ~ n o t ~ t h e ~ u s u a l ~}$ convergence of the $H^{2}$-norm of $y-y^{*}$ taken on $(0, L)$ as $y$ and $y^{*}$ do not belong to $H^{2}(0, L)$. This definition enables to define an exponential stability in $H^{2}$-norm for a function that has a discontinuity at some point and is regular elsewhere. Note that, the convergence to 0 of the $H^{2}{ }^{-n o r m}$ in the usual sense does not ensure the convergence of the shock location $x_{s}$ to $x_{0}$. Thus, to guarantee that the state converges to the shock steady state, we have to take account of the shock location, which is explained in Definition 2.1.

Remark 3. Note that this definition of exponential stability only deals a priori with $t \in[0, T)$ for any $T>0$. However this, together with Theorem 2.1 implies the global existence in time of the solution $\left(y, x_{s}\right)$ and the exponential stability on $[0,+\infty)$. This is shown at the end of the proof of Theorem 4.1.

We can now state the main result of this paper

Theorem 2.2. Let $\gamma>0$. If the following conditions hold:

$$
\begin{aligned}
& b_{1} \in\left(\gamma e^{-\gamma x_{0}}, \frac{\gamma e^{-\gamma x_{0}}}{1-e^{-\gamma x_{0}}}\right), b_{2} \in\left(\gamma e^{-\gamma\left(L-x_{0}\right)}, \frac{\gamma e^{-\gamma\left(L-x_{0}\right)}}{1-e^{-\gamma\left(L-x_{0}\right)}}\right), \\
& k_{1}^{2}<e^{-\gamma x_{0}}\left(1-\frac{b_{1}}{\gamma}\left(b_{1} \frac{1-e^{-\gamma x_{0}}}{\gamma e^{-\gamma x_{0}}}+b_{2} \frac{1-e^{-\gamma\left(L-x_{0}\right)}}{\gamma e^{-\gamma\left(L-x_{0}\right)}}\right)\right), \\
& k_{2}^{2}<e^{-\gamma\left(L-x_{0}\right)}\left(1-\frac{b_{2}}{\gamma}\left(b_{1} \frac{1-e^{-\gamma x_{0}}}{\gamma e^{-\gamma x_{0}}}+b_{2} \frac{1-e^{-\gamma\left(L-x_{0}\right)}}{\gamma e^{-\gamma\left(L-x_{0}\right)}}\right)\right),
\end{aligned}
$$

then the steady state $\left(y^{*}, x_{0}\right)$ of the system (1), (3), (4), (8) is exponentially stable for the $H^{2}{ }^{-}$norm with decay rate $\gamma / 4$.

The proof of this theorem is given in Section 4. 
Remark 4. One can actually check that for any $\gamma>0$ there exist parameters $b_{1}, b_{2}$ and $k_{1}, k_{2}$ satisfying (16) as, for $b_{1}=\gamma e^{-\gamma x_{0}}$ and $b_{2}=\gamma e^{-\gamma\left(L-x_{0}\right)}$, one has

$$
\begin{aligned}
& 1-\frac{b_{1}}{\gamma}\left(b_{1} \frac{1-e^{-\gamma x_{0}}}{\gamma e^{-\gamma x_{0}}}+b_{2} \frac{1-e^{-\gamma\left(L-x_{0}\right)}}{\gamma e^{-\gamma\left(L-x_{0}\right)}}\right) \\
= & 1-e^{-\gamma x_{0}}\left(2-e^{-\gamma x_{0}}-e^{-\gamma\left(L-x_{0}\right)}\right) \\
= & e^{-2 \gamma x_{0}}\left(e^{\gamma x_{0}}-1\right)^{2}+e^{-\gamma L}>0 .
\end{aligned}
$$

Similarly, we get

$$
\begin{aligned}
& 1-\frac{b_{2}}{\gamma}\left(b_{1} \frac{1-e^{-\gamma x_{0}}}{\gamma e^{-\gamma x_{0}}}+b_{2} \frac{1-e^{-\gamma\left(L-x_{0}\right)}}{\gamma e^{-\gamma\left(L-x_{0}\right)}}\right) \\
= & e^{-2 \gamma\left(L-x_{0}\right)}\left(e^{\gamma\left(L-x_{0}\right)}-1\right)^{2}+e^{-\gamma L}>0 .
\end{aligned}
$$

Therefore, by continuity, there exist $b_{1}$ and $b_{2}$, satisfying condition (16a) such that there exist $k_{1}$ and $k_{2}$ satisfying $16 \mathrm{~b}$ and $16 \mathrm{c}$. This implies that $\gamma$ can be made arbitrarily large. And, from (16a)-16c), we can note that for large $\gamma$ the conditions on the $k_{i}$ tend to

$$
k_{1}^{2}<e^{-\gamma x_{0}}, \quad k_{2}^{2}<e^{-\gamma\left(L-x_{0}\right)} .
$$

Remark 5. The result can also be generalized to $H^{k}$-norm for any integer $k \geqslant 2$ in the sense of Definition 2.1 by replacing $H^{2}$ with $H^{k}$. This can be easily done by just adapting the Lyapunov function defined below by (31)-(37) as was done in [3, Sections 4.5 and 6.2].

Remark 6. If we set $k_{1}=k_{2}=b_{1}=b_{2}=0$, then from (8), $u_{0}(t) \equiv 1$ and $u_{L}(t) \equiv-1$. Thus it seems logical that the larger $\gamma$ is, the smaller $k_{1}$ and $k_{2}$ are. However, it could seem counter-intuitive that $b_{1}$ and $b_{2}$ have to tend to 0 when $\gamma$ tends to $+\infty$, as if one sets $b_{1}=0$ and $b_{2}=0$, one cannot stabilize the location of the system just like in the constant open-loop control case. In other words for any $\gamma>0$ the prescribed feedback works while the limit feedback we obtain by letting $\gamma \rightarrow+\infty$ cannot even ensure the asymptotic stability of the system. The explanation behind this apparent paradox is that when $\gamma$ tends to infinity, the Lyapunov function candidate used to prove Theorem 4.1 is not equivalent to the norm of the solution and cannot guarantee anymore the exponential decay of the solution in the $H^{2}$-norm. More precisely, one can see, looking at (97) and (99), that the hypothesis (44) of Lemma 4.1 does not hold anymore.

\section{An equivalent system with shock-free solutions}

Our strategy to analyze the existence and the exponential stability of the shock wave solutions to the scalar Burgers equation (1) is to use an equivalent $2 \times 2$ quasilinear hyperbolic system having shock-free solutions. In order to set up this equivalent system, we define the two following functions

$$
y_{1}(t, x)=y\left(t, x \frac{x_{s}(t)}{x_{0}}\right), \quad y_{2}(t, x)=y\left(t, L+x \frac{x_{s}(t)-L}{x_{0}}\right)
$$

and the new state variables as follows:

$$
\mathbf{z}(t, x)=\left(\begin{array}{l}
z_{1}(t, x) \\
z_{2}(t, x)
\end{array}\right)=\left(\begin{array}{l}
y_{1}(t, x)-1 \\
y_{2}(t, x)+1
\end{array}\right), x \in\left(0, x_{0}\right) .
$$

The idea behind the definition of $y_{1}, y_{2}$ is to describe the behavior of the solution $y(t, x)$ before and after the moving shock, while studying functions on a time invariant interval. Observe indeed that the functions $y_{1}$ and $y_{2}$ in $(19)$ correspond to the solution $y(t, x)$ on the time varying intervals $\left(0, x_{s}(t)\right)$ 
and $\left(x_{s}(t), L\right)$ respectively, albeit with a time varying scaling of the space coordinate $x$ which is driven by $x_{s}(t)$ and allows to define the new state variables $\left(z_{1}, z_{2}\right)$ on the fixed time invariant interval $\left(0, x_{0}\right)$. The reason to rescale $y_{2}$ on $\left(0, x_{0}\right)$ instead of $\left(x_{0}, L\right)$ is to simplify the analysis by defining state variables on the same space interval with the same direction of propagation.

Besides, from (20), the former steady state $\left(y^{*}, x_{0}\right)$ corresponds now to the steady state $(\mathbf{z}=$ $\left.\mathbf{0}, x_{s}=x_{0}\right)$ in the new variables. With these new variables, the dynamics of $\left(y, x_{s}\right)$ can now be expressed as follows:

$$
\begin{aligned}
& z_{1 t}+\left(1+z_{1}-x \frac{\dot{x}_{s}}{x_{0}}\right) z_{1 x} \frac{x_{0}}{x_{s}}=0, \\
& z_{2 t}+\left(1-z_{2}+x \frac{\dot{x}_{s}}{x_{0}}\right) z_{2 x} \frac{x_{0}}{L-x_{s}}=0, \\
& \dot{x}_{s}(t)=\frac{z_{1}\left(t, x_{0}\right)+z_{2}\left(t, x_{0}\right)}{2},
\end{aligned}
$$

with the boundary conditions:

$$
\begin{aligned}
& z_{1}(t, 0)=k_{1} z_{1}\left(t, x_{0}\right)+b_{1}\left(x_{0}-x_{s}(t)\right), \\
& z_{2}(t, 0)=k_{2} z_{2}\left(t, x_{0}\right)+b_{2}\left(x_{0}-x_{s}(t)\right),
\end{aligned}
$$

and initial condition

$$
\mathbf{z}(0, x)=\mathbf{z}^{0}(x), \quad x_{s}(0)=x_{s 0},
$$

where $\mathbf{z}^{0}=\left(z_{1}^{0}, z_{2}^{0}\right)^{T}$ and

$$
\begin{aligned}
z_{1}^{0}(x) & =y_{0}\left(x \frac{x_{s 0}}{x_{0}}\right)-1 \\
z_{2}^{0}(x) & =y_{0}\left(L+x \frac{x_{s 0}-L}{x_{0}}\right)+1
\end{aligned}
$$

Furthermore, in the new variables, the compatibility conditions $(9-10$ are expressed as follows:

$$
\begin{aligned}
& z_{1}^{0}(0)=k_{1} z_{1}^{0}\left(x_{0}\right)+b_{1}\left(x_{0}-x_{s 0}\right), \\
& z_{2}^{0}(0)=k_{2} z_{2}^{0}\left(x_{0}\right)+b_{2}\left(x_{0}-x_{s 0}\right),
\end{aligned}
$$

and

$$
\begin{aligned}
& \left(1+z_{1}^{0}(0)\right) z_{1 x}^{0}(0) \frac{x_{0}}{x_{s 0}}=k_{1}\left(1+z_{1}^{0}\left(x_{0}\right)-\frac{z_{1}^{0}\left(x_{0}\right)+z_{2}^{0}\left(x_{0}\right)}{2}\right) z_{1 x}^{0}\left(x_{0}\right) \frac{x_{0}}{x_{s 0}}+b_{1} \frac{z_{1}^{0}\left(x_{0}\right)+z_{2}^{0}\left(x_{0}\right)}{2}, \\
& \left(1-z_{2}^{0}(0)\right) z_{2 x}^{0}(0) \frac{x_{0}}{L-x_{s 0}}=k_{2}\left(1-z_{2}^{0}\left(x_{0}\right)+\frac{z_{1}^{0}\left(x_{0}\right)+z_{2}^{0}\left(x_{0}\right)}{2}\right) z_{2 x}^{0}\left(x_{0}\right) \frac{x_{0}}{L-x_{s 0}}+b_{2} \frac{z_{1}^{0}\left(x_{0}\right)+z_{2}^{0}\left(x_{0}\right)}{2} .
\end{aligned}
$$

Concerning the existence and uniqueness of the solution to the system $(21)-(23)$, we have the following lemma.

Lemma 3.1. For all $T>0$, there exists $\delta(T)>0$ such that, for every $x_{s 0} \in(0, L)$ and $\mathbf{z}^{0} \in$ $H^{2}\left(\left(0, x_{0}\right) ; \mathbb{R}^{2}\right)$ satisfying the compatibility conditions 25$)-26$ and

$$
\left|\mathbf{z}^{0}\right|_{H^{2}\left(\left(0, x_{0}\right) ; \mathbb{R}^{2}\right)} \leqslant \delta(T), \quad\left|x_{s 0}-x_{0}\right| \leqslant \delta(T),
$$

the system 21) - 23) has a unique classical solution $\left(\mathbf{z}, x_{s}\right) \in C^{0}\left([0, T] ; H^{2}\left(\left(0, x_{0}\right) ; \mathbb{R}^{2}\right)\right) \times C^{1}([0, T] ;(0, L))$. Moreover, there exists $C(T)$ such that the following estimate holds for all $t \in[0, T]$

$$
|\mathbf{z}(t, \cdot)|_{H^{2}\left(\left(0, x_{0}\right) ; \mathbb{R}^{2}\right)}+\left|x_{s}(t)-x_{0}\right| \leqslant C(T)\left(\left|\mathbf{z}^{0}\right|_{H^{2}\left(\left(0, x_{0}\right) ; \mathbb{R}^{2}\right)}+\left|x_{s 0}-x_{0}\right|\right) .
$$


Proof. The proof of Lemma 3.1 is given in Appendix A

From this lemma, it is then clear that the proof of Theorem 2.1 follows immediately.

Proof of Theorem 2.1. The change of variables (19), 201 induces an equivalence between the classical solutions $\left(\mathbf{z}, x_{s}\right)$ of the system (21)-23) and the entropy solutions with a single shock $\left(y, x_{s}\right)$ of the system (1)-(4), (6), (8). Consequently, from (20) and provided $\left|\mathbf{z}^{0}\right|_{H^{2}\left(\left(0, x_{0}\right) ; \mathbb{R}^{2}\right)}$ and $\left|x_{s 0}-x_{0}\right|$ are sufficiently small, the existence and uniqueness of a solution with a single shock $\left(y, x_{s}\right)$ to the system (1)-(4), (6), (8) satisfying the entropy condition (5) when $\left(y_{0}, x_{s 0}\right)$ is in a sufficiently small neighborhood of $\left(y^{*}, x_{0}\right)$, follows directly from the existence and uniqueness of the classical solution $\left(\mathbf{z}, x_{s}\right)$ to the system (21)- 23$)$ which is guaranteed by Lemma 3.1

Remark 7. Under the assumption in Lemma 3.1. if we assume furthermore that $\mathbf{z}^{0} \in H^{k}\left(\left(0, x_{0}\right) ; \mathbb{R}^{2}\right)$ with $k \geqslant 2$ satisfying the $k$-th order compatibility conditions (see the definition in [3, p.143]), then $\left(\mathbf{z}, x_{s}\right) \in C^{0}\left([0, T] ; H^{k}\left(\left(0, x_{0}\right) ; \mathbb{R}^{2}\right)\right) \times C^{k}([0, T] ; \mathbb{R})$ and $[28$ still holds. This is a straightforward extension of the proof in Appendix A, thus we will not give the details of this proof here.

\section{Exponential stability for the $H^{2}$-norm}

This section is devoted to the proof of Theorem 2.2 concerning the exponential stability of the steady state of system (1), (3), (4), (8). Actually, on the basis of the change of variables introduced in the previous section, we know that we only have to prove the exponential stability of the steady state of the auxiliary system (21)- 22 according to the following theorem which is equivalent to Theorem 2.2 .

Theorem 4.1. For any $\gamma>0$, if condition (16) on the parameters of the feedback holds, then there exist $\delta^{*}>0$ and $C>0$ such that for any $\mathbf{z}^{0} \in H^{2}\left(\left(0, x_{0}\right) ; \mathbb{R}^{2}\right)$ and $x_{s 0} \in(0, L)$ satisfying

$$
\left|\mathbf{z}^{0}\right|_{H^{2}\left(\left(0, x_{0}\right) ; \mathbb{R}^{2}\right)} \leqslant \delta^{*}, \quad\left|x_{s 0}-x_{0}\right| \leqslant \delta^{*}
$$

and the compatibility conditions (25)-(26), and for any $T>0$ the system (21) $-(23)$ has a unique classical solution $\left(\mathbf{z}, x_{s}\right) \in C^{0}\left([0, T] ; H^{2}\left(\left(0, x_{0}\right) ; \mathbb{R}^{2}\right)\right) \times C^{1}([0, T] ; \mathbb{R})$ such that

$$
|\mathbf{z}(t, \cdot)|_{H^{2}\left(\left(0, x_{0}\right) ; \mathbb{R}^{2}\right)}+\left|x_{s}(t)-x_{0}\right| \leqslant C e^{-\gamma t / 4}\left(\left|\mathbf{z}^{0}\right|_{H^{2}\left(\left(0, x_{0}\right) ; \mathbb{R}^{2}\right)}+\left|x_{s 0}-x_{0}\right|\right), \quad \forall t \in[0, T) .
$$

When this theorem holds, we say that the steady state $\left(\mathbf{z}=\mathbf{0}, x_{s}=x_{0}\right)$ of the system $21--22$ is exponentially stable for the $H^{2}$-norm with convergence rate $\gamma / 4$. Recall that, from Remark 4 there always exist parameters such that (16) holds.

Before proving Theorem 4.1, let us give an overview of our strategy. We first introduce a Lyapunov function candidate $V$ with parameters to be chosen. Then, in Lemma 4.1, we give a condition on the parameters such that $V$ is equivalent to the square of the $H^{2}$-norm of $\mathbf{z}$ plus the absolute value of $x_{s}-x_{0}$, which implies that proving the exponential decay of $V$ with rate $\gamma / 2$ is enough to show the exponential stability of the system with decay rate $\gamma / 4$ for the $H^{2}$-norm. In Lemma 4.2 , we show that in order to obtain Theorem 4.1, it is enough to prove that $V$ decays along any solutions $\left(\mathbf{z}, x_{s}\right) \in C^{3}\left([0, T] \times\left[0, x_{0}\right] ; \mathbb{R}^{2}\right) \times C^{3}([0, T] ; \mathbb{R})$ with a density argument. Then in Lemma 4.3 , we compute the time derivative of $V$ along any $C^{3}$ solutions of the system and we give a sufficient condition on the parameters such that $V$ satisfies a useful estimate along these solutions. Finally, we show that there exist parameters satisfying the sufficient condition of Lemma 4.3. This, together with Lemma 4.2, ends the proof of Theorem 4.1.

We now introduce the following candidate Lyapunov function which is defined for all $\mathbf{z}=\left(z_{1}, z_{2}\right)^{T} \in$ $H^{2}\left(\left(0, x_{0}\right) ; \mathbb{R}^{2}\right)$ and $x_{s} \in(0, L)$ :

$$
V\left(\mathbf{z}, x_{s}\right)=V_{1}(\mathbf{z})+V_{2}\left(\mathbf{z}, x_{s}\right)+V_{3}\left(\mathbf{z}, x_{s}\right)+V_{4}\left(\mathbf{z}, x_{s}\right)+V_{5}\left(\mathbf{z}, x_{s}\right)+V_{6}\left(\mathbf{z}, x_{s}\right)
$$

with

$$
\begin{aligned}
V_{1}(\mathbf{z}) & =\int_{0}^{x_{0}} p_{1} e^{\frac{-\mu x}{\eta_{1}}} z_{1}^{2}+p_{2} e^{\frac{-\mu x}{\eta_{2}}} z_{2}^{2} d x, \\
V_{2}\left(\mathbf{z}, x_{s}\right) & =\int_{0}^{x_{0}} p_{1} e^{\frac{-\mu x}{\eta_{1}}} z_{1 t}^{2}+p_{2} e^{\frac{-\mu x}{\eta_{2}}} z_{2 t}^{2} d x,
\end{aligned}
$$




$$
\begin{gathered}
V_{3}\left(\mathbf{z}, x_{s}\right)=\int_{0}^{x_{0}} p_{1} e^{\frac{-\mu x}{\eta_{1}}} z_{1 t t}^{2}+p_{2} e^{\frac{-\mu x}{\eta_{2}}} z_{2 t t}^{2} d x \\
V_{4}\left(\mathbf{z}, x_{s}\right)=\int_{0}^{x_{0}} \bar{p}_{1} e^{\frac{-\mu x}{\eta_{1}}} z_{1}\left(x_{s}-x_{0}\right) d x+\int_{0}^{x_{0}} \bar{p}_{2} e^{\frac{-\mu x}{\eta_{2}}} z_{2}\left(x_{s}-x_{0}\right) d x+\kappa\left(x_{s}-x_{0}\right)^{2}, \\
V_{5}\left(\mathbf{z}, x_{s}\right)=\int_{0}^{x_{0}} \bar{p}_{1} e^{\frac{-\mu x}{\eta_{1}}} z_{1 t} \dot{x}_{s} d x+\int_{0}^{x_{0}} \bar{p}_{2} e^{\frac{-\mu x}{\eta_{2}}} z_{2 t} \dot{x}_{s} d x+\kappa\left(\dot{x}_{s}\right)^{2}, \\
V_{6}\left(\mathbf{z}, x_{s}\right)=\int_{0}^{x_{0}} \bar{p}_{1} e^{\frac{-\mu x}{\eta_{1}}} z_{1 t t} \ddot{x}_{s} d x+\int_{0}^{x_{0}} \bar{p}_{2} e^{\frac{-\mu x}{\eta_{2}}} z_{2 t t} \ddot{x}_{s} d x+\kappa\left(\ddot{x}_{s}\right)^{2} .
\end{gathered}
$$

In (32)-(37), $\mu, p_{1}, p_{2}, \bar{p}_{1}, \bar{p}_{2}$ are positive constants. Moreover

$$
\eta_{1}=1, \quad \eta_{2}=\frac{x_{0}}{L-x_{0}}
$$

and

$$
\kappa>1 .
$$

Actually, in this section, we will need to evaluate $V\left(\mathbf{z}, x_{s}\right)$ only along the system solutions for which the variables $\mathbf{z}_{t}=\left(z_{1 t}, z_{2 t}\right), \mathbf{z}_{t t}=\left(z_{1 t t}, z_{2 t t}\right), \dot{x}_{s}$ and $\ddot{x}_{s}$ that appear in the definition of $V$ can be well defined as functions of $\left(\mathbf{z}, x_{s}\right) \in H^{2}\left(\left(0, x_{0}\right) ; \mathbb{R}^{2}\right) \times(0, L)$ from the system $212-(22)$ and their space derivatives. For example, $z_{1 t}$ and $z_{2 t}$ are defined as functions of $\left(\mathbf{z}, x_{s}\right)$ by

$$
\begin{aligned}
& z_{1 t}:=-\left(1+z_{1}-x \frac{z_{1}\left(x_{0}\right)+z_{2}\left(x_{0}\right)}{2 x_{0}}\right) z_{1 x} \frac{x_{0}}{x_{s}}, \\
& z_{2 t}:=-\left(1-z_{2}+x \frac{z_{1}\left(x_{0}\right)+z_{2}\left(x_{0}\right)}{2 x_{0}}\right) z_{2 x} \frac{x_{0}}{L-x_{s}},
\end{aligned}
$$

and $z_{1 t t}$ and $z_{2 t t}$ as functions of $\left(\mathbf{z}, x_{s}\right)$ by

$$
\begin{gathered}
z_{1 t t}:=-\left(1+z_{1}-x \frac{z_{1}\left(x_{0}\right)+z_{2}\left(x_{0}\right)}{2 x_{0}}\right)\left(z_{1 t}\right)_{x} \frac{x_{0}}{x_{s}} \\
-\left(z_{1 t}-x \frac{z_{1 t}\left(x_{0}\right)+z_{2 t}\left(x_{0}\right)}{2 x_{0}}\right) z_{1 x} \frac{x_{0}}{x_{s}}-z_{1 t} \frac{z_{1}\left(x_{0}\right)+z_{2}\left(x_{0}\right)}{2 x_{s}}, \\
\begin{array}{r}
z_{2 t t}:=-\left(1-z_{2}+x \frac{z_{1}\left(x_{0}\right)+z_{2}\left(x_{0}\right)}{2 x_{0}}\right)\left(z_{2 t}\right)_{x} \frac{x_{0}}{L-x_{s}} \\
+\left(z_{2 t}-x \frac{z_{1 t}\left(x_{0}\right)+z_{2 t}\left(x_{0}\right)}{2 x_{0}}\right) z_{2 x} \frac{x_{0}}{L-x_{s}}+z_{2 t} \frac{z_{1}\left(x_{0}\right)+z_{2}\left(x_{0}\right)}{2\left(L-x_{s}\right)} .
\end{array}
\end{gathered}
$$

The functions $z_{1 t}$ and $z_{2 t}$ which appear in (42) and (43) are supposed to be defined by (40) and (41) respectively.

Remark 8. When looking for a Lyapunov function to stabilize the state $\left(z_{1}, z_{2}\right)$ in $H^{2}$-norm, the component $\left(V_{1}+V_{2}+V_{3}\right)$ can be seen as the most natural and easiest choice, as it is equivalent to a weighted $\mathrm{H}^{2}$-norm by properly choosing the parameters. This kind of Lyapunov function, sometimes called basic quadratic Lyapunov function, is used for instance in [2] or [3, Section 4.4]. However, in the present case one needs to stabilize both the state $\mathbf{z}$ and the shock location $x_{s}$, which requires to add additional terms to the Lyapunov function in order to deal with $x_{s}$. Besides, as we have no direct control on $x_{s}$ (observe that none of the terms of the right-hand side of (4), or equivalently of the third equation of (21), is a control), we need to add some coupling terms between the state $\mathbf{z}$ on which we have a control and the shock location $x_{s}$ in the Lyapunov function. Thus, $V_{4}$ is designed to provide such coupling with the product of the component of $\mathbf{z}$ and $x_{s}$, while $V_{5}$ and $V_{6}$ are its analogous for the time derivatives terms (as $V_{2}$ and $V_{3}$ are the analogous of $V_{1}$ respectively for the first and second time derivative of $\mathbf{z}$ ).

We now state the following lemma, providing a condition on $\mu, p_{1}, p_{2}, \bar{p}_{1}$ and $\bar{p}_{2}$ such that $V\left(\mathbf{z}, x_{s}\right)$ is equivalent to $\left(|\mathbf{z}|_{H^{2}\left(\left(0, x_{0}\right) ; \mathbb{R}^{2}\right)}^{2}+\left|x_{s}-x_{0}\right|^{2}\right)$. 
Lemma 4.1. If

$$
\max \left(\Theta_{1}, \Theta_{2}\right)<2
$$

where

$$
\Theta_{1}:=\frac{\bar{p}_{1}^{2}}{p_{1}} \frac{\eta_{1}}{\mu}\left(1-e^{\frac{-\mu x_{0}}{\eta_{1}}}\right), \quad \Theta_{2}:=\frac{\bar{p}_{2}^{2}}{p_{2}} \frac{\eta_{2}}{\mu}\left(1-e^{-\frac{\mu x_{0}}{\eta_{2}}}\right),
$$

there exists $\beta>0$ such that

$$
\beta\left(|\mathbf{z}|_{H^{2}\left(\left(0, x_{0}\right) ; \mathbb{R}^{2}\right)}^{2}+\left|x_{s}-x_{0}\right|^{2}\right) \leqslant V \leqslant \frac{1}{\beta}\left(|\mathbf{z}|_{H^{2}\left(\left(0, x_{0}\right) ; \mathbb{R}^{2}\right)}^{2}+\left|x_{s}-x_{0}\right|^{2}\right)
$$

for any $\left(\mathbf{z}, x_{s}\right) \in H^{2}\left(\left(0, x_{0}\right) ; \mathbb{R}^{2}\right) \times(0, L)$ satisfying

$$
|\mathbf{z}|_{H^{2}\left(\left(0, x_{0}\right) ; \mathbb{R}^{2}\right)}^{2}+\left|x_{s}-x_{0}\right|^{2}<\beta^{2} .
$$

Proof of Lemma 4.1. Let us start with

$$
V_{4}=\int_{0}^{x_{0}} \bar{p}_{1} e^{\frac{-\mu x}{\eta_{1}}} z_{1}\left(x_{s}-x_{0}\right) d x+\int_{0}^{x_{0}} \bar{p}_{2} e^{\frac{-\mu x}{\eta_{2}}} z_{2}\left(x_{s}-x_{0}\right) d x+\kappa\left(x_{s}-x_{0}\right)^{2} .
$$

Using Young's inequality we get

$$
\begin{aligned}
& -\frac{1}{2}\left(\int_{0}^{x_{0}} \bar{p}_{1} e^{\frac{-\mu x}{\eta_{1}}} z_{1} d x\right)^{2}-\frac{\left(x_{s}-x_{0}\right)^{2}}{2}-\frac{1}{2}\left(\int_{0}^{x_{0}} \bar{p}_{2} e^{\frac{-\mu x}{\eta_{2}}} z_{2} d x\right)^{2}-\frac{\left(x_{s}-x_{0}\right)^{2}}{2} \\
& +\kappa\left(x_{s}-x_{0}\right)^{2} \leqslant V_{4} \leqslant \frac{1}{2}\left(\int_{0}^{x_{0}} \bar{p}_{1} e^{\frac{-\mu x}{\eta_{1}}} z_{1} d x\right)^{2} \\
& +\frac{\left(x_{s}-x_{0}\right)^{2}}{2}+\frac{1}{2}\left(\int_{0}^{x_{0}} \bar{p}_{2} e^{\frac{-\mu x}{\eta_{2}}} z_{2} d x\right)^{2}+\frac{\left(x_{s}-x_{0}\right)^{2}}{2}+\kappa\left(x_{s}-x_{0}\right)^{2} .
\end{aligned}
$$

Hence, using the Cauchy-Schwarz inequality and the expression of $V_{1}$ given in 32 ,

$$
\begin{aligned}
& p_{1}\left(1-\frac{1}{2} \Theta_{1}\right) \int_{0}^{x_{0}} e^{\frac{-\mu x}{\eta_{1}}} z_{1}^{2} d x+p_{2}\left(1-\frac{1}{2} \Theta_{2}\right) \int_{0}^{x_{0}} e^{\frac{-\mu x}{\eta_{2}}} z_{2}^{2} d x \\
& +\left(x_{s}-x_{0}\right)^{2}(\kappa-1) \leqslant V_{1}+V_{4} \leqslant p_{1}\left(1+\frac{1}{2} \Theta_{1}\right) \int_{0}^{x_{0}} e^{\frac{-\mu x}{\eta_{1}}} z_{1}^{2} d x \\
& +p_{2}\left(1+\frac{1}{2} \Theta_{2}\right) \int_{0}^{x_{0}} e^{\frac{-\mu x}{\eta_{2}}} z_{2}^{2} d x+\left(x_{s}-x_{0}\right)^{2}(\kappa+1),
\end{aligned}
$$

and similarly

$$
\begin{aligned}
& p_{1}\left(1-\frac{1}{2} \Theta_{1}\right) \int_{0}^{x_{0}} e^{\frac{-\mu x}{\eta_{1}}} z_{1 t}^{2} d x+p_{2}\left(1-\frac{1}{2} \Theta_{2}\right) \int_{0}^{x_{0}} e^{\frac{-\mu x}{\eta_{2}}} z_{2 t}^{2} d x \\
& +\left(\dot{x}_{s}\right)^{2}(\kappa-1) \leqslant V_{2}+V_{5} \leqslant p_{1}\left(1+\frac{1}{2} \Theta_{1}\right) \int_{0}^{x_{0}} e^{\frac{-\mu x}{\eta_{1}}} z_{1 t}^{2} d x \\
& +p_{2}\left(1+\frac{1}{2} \Theta_{2}\right) \int_{0}^{x_{0}} e^{\frac{-\mu x}{\eta_{2}}} z_{2 t}^{2} d x+\left(\dot{x}_{s}\right)^{2}(\kappa+1),
\end{aligned}
$$

and also

$$
\begin{aligned}
& p_{1}\left(1-\frac{1}{2} \Theta_{1}\right) \int_{0}^{x_{0}} e^{\frac{-\mu x}{\eta_{1}}} z_{1 t t}^{2} d x+p_{2}\left(1-\frac{1}{2} \Theta_{2}\right) \int_{0}^{x_{0}} e^{\frac{-\mu x}{\eta_{2}}} z_{2 t t}^{2} d x \\
& +\left(\ddot{x}_{s}\right)^{2}(\kappa-1) \leqslant V_{3}+V_{6} \leqslant p_{1}\left(1+\frac{1}{2} \Theta_{1}\right) \int_{0}^{x_{0}} e^{\frac{-\mu x}{\eta_{1}}} z_{1 t t}^{2} d x \\
& +p_{2}\left(1+\frac{1}{2} \Theta_{2}\right) \int_{0}^{x_{0}} e^{\frac{-\mu x}{\eta_{2}}} z_{2 t t}^{2} d x+\left(\ddot{x}_{s}\right)^{2}(\kappa+1) .
\end{aligned}
$$


Hence, from (39), $\kappa>1$ and (44) is satisfied, there exists $\sigma>0$ such that

$$
\sigma\left(|\mathbf{z}|_{H_{t}^{2}\left(\left(0, x_{0}\right) ; \mathbb{R}^{2}\right)}^{2}+\left|x_{s}-x_{0}\right|^{2}\right) \leqslant V \leqslant \frac{1}{\sigma}\left(|\mathbf{z}|_{H_{t}^{2}\left(\left(0, x_{0}\right) ; \mathbb{R}^{2}\right)}^{2}+\left|x_{s}-x_{0}\right|^{2}\right),
$$

where, for a function $\mathbf{z} \in H^{2}\left(\left(0, x_{0}\right) ; \mathbb{R}^{2}\right),|\mathbf{z}|_{H_{t}^{2}\left(\left(0, x_{0}\right) ; \mathbb{R}^{2}\right)}$ is defined by

$$
|\mathbf{z}|_{H_{t}^{2}\left(\left(0, x_{0}\right) ; \mathbb{R}^{2}\right)}=\left(|\mathbf{z}|_{L^{2}\left(\left(0, x_{0}\right) ; \mathbb{R}^{2}\right)}^{2}+\left|\mathbf{z}_{t}\right|_{L^{2}\left(\left(0, x_{0}\right) ; \mathbb{R}^{2}\right)}^{2}+\left|\mathbf{z}_{t t}\right|_{L^{2}\left(\left(0, x_{0}\right) ; \mathbb{R}^{2}\right)}^{2}\right)^{1 / 2},
$$

with $\mathbf{z}_{t}$ and $\mathbf{z}_{t t}$ defined as $40-43$. Let us point out that from 40-43), there exists $C>0$ such that

$$
\frac{1}{C}|\mathbf{z}|_{H^{2}\left(\left(0, x_{0}\right) ; \mathbb{R}^{2}\right)} \leqslant|\mathbf{z}|_{H_{t}^{2}\left(\left(0, x_{0}\right) ; \mathbb{R}^{2}\right)} \leqslant C|\mathbf{z}|_{H^{2}\left(\left(0, x_{0}\right) ; \mathbb{R}^{2}\right)},
$$

if $\left(|\mathbf{z}|_{H^{2}\left(\left(0, x_{0}\right) ; \mathbb{R}^{2}\right)}^{2}+\left|x_{s}-x_{0}\right|^{2}\right)<1 / C$. It follows from (53) and 55 that $\beta>0$ can be taken sufficiently small such that inequality 46 holds provided (47) is satisfied. This concludes the proof of Lemma 4.1

Before proving Theorem 4.1, we introduce the following density argument, which shows that it is enough to prove the exponential decay of $V$ along any $C^{3}$ solutions of the system.

Lemma 4.2. Let $V$ be $a C^{1}$ and nonnegative functional on $C^{0}\left([0, T] ; H^{2}\left(\left(0, x_{0}\right) ; \mathbb{R}^{2}\right)\right) \times C^{1}([0, T] ; \mathbb{R})$. If there exist $\delta>0$ and $\gamma>0$ such that for any $\left.\left(\mathbf{z}, x_{s}\right) \in C^{3}\left([0, T] \times\left[0, x_{0}\right] ; \mathbb{R}^{2}\right)\right) \times C^{3}([0, T] ; \mathbb{R})$ solution of 21] -22 , with associated initial condition $\left(\mathbf{z}^{0}, x_{s 0}\right)$ satisfying $\left|\mathbf{z}^{0}\right|_{H^{2}\left(\left(0, x_{0}\right) ; \mathbb{R}^{2}\right)} \leqslant \delta$ and $\left|x_{s 0}-x_{0}\right| \leqslant \delta$, one has

$$
\frac{d V\left(\mathbf{z}(t, \cdot), x_{s}(t)\right)}{d t} \leqslant-\frac{\gamma}{2} V\left(\mathbf{z}(t, \cdot), x_{s}(t)\right)
$$

then (56) also holds in a distribution sense for any $\left(\mathbf{z}, x_{s}\right) \in C^{0}\left([0, T] ; H^{2}\left(\left(0, x_{0}\right) ; \mathbb{R}^{2}\right)\right) \times C^{1}([0, T] ; \mathbb{R})$ solution of 21] -22 such that the associated initial condition $\left(\mathbf{z}^{0}, x_{s 0}\right)$ satisfies $\left|\mathbf{z}^{0}\right|_{H^{2}\left(\left(0, x_{0}\right) ; \mathbb{R}^{2}\right)}<\delta$ and $\left|x_{s 0}-x_{0}\right|<\delta$.

Proof of Lemma 4.2. Let $V$ be a $C^{1}$ and nonnegative functional on $C^{0}\left([0, T] ; H^{2}\left(\left(0, x_{0}\right) ; \mathbb{R}^{2}\right)\right) \times C^{1}([0, T] ; \mathbb{R})$ and let $\left(\mathbf{z}, x_{s}\right) \in C^{0}\left([0, T] ; H^{2}\left(\left(0, x_{0}\right) ; \mathbb{R}^{2}\right)\right) \times C^{1}([0, T] ; \mathbb{R})$ be solution of $(21)-(22)$ with associated initial condition $\left|\mathbf{z}^{0}\right|_{H^{2}\left(\left(0, x_{0}\right) ; \mathbb{R}^{2}\right)} \leqslant \delta$ and $\left|x_{s 0}-x_{0}\right| \leqslant \delta$. Let $\left(\mathbf{z}^{0 n}, x_{s 0}^{n}\right) \in H^{4}\left(\left(0, x_{0}\right) ; \mathbb{R}^{2}\right) \times(0, L), n \in \mathbb{N}$ be a sequence of functions that satisfy the fourth order compatibility conditions and

$$
\left|\mathbf{z}^{0 n}\right|_{H^{2}\left(\left(0, x_{0}\right) ; \mathbb{R}^{2}\right)} \leqslant \delta, \quad\left|x_{s 0}^{n}-x_{0}\right| \leqslant \delta,
$$

such that $\mathbf{z}^{0 n}$ converges to $\mathbf{z}^{0}$ in $H^{2}\left(\left(0, x_{0}\right) ; \mathbb{R}^{2}\right)$ and $x_{s 0}^{n}$ converges to $x_{s 0}$. From Remark 7 , there exists a unique solution $\left(\mathbf{z}^{n}, x_{s}^{n}\right) \in C^{0}\left([0, T] ; H^{4}\left(\left(0, x_{0}\right) ; \mathbb{R}^{2}\right)\right) \times C^{4}([0, T] ; \mathbb{R})$ to 21$]-22$ corresponding to the initial condition $\left(\mathbf{z}^{0 n}, x_{s 0}^{n}\right)$ and for any $t \in[0, T]$, we have

$$
\left|\mathbf{z}^{n}(t, \cdot)\right|_{H^{2}\left(\left(0, x_{0}\right) ; \mathbb{R}^{2}\right)}+\left|x_{s}^{n}(t)-x_{0}\right| \leqslant C(T)\left(\left|\mathbf{z}^{0 n}\right|_{H^{2}\left(\left(0, x_{0}\right) ; \mathbb{R}^{2}\right)}+\left|x_{s 0}^{n}-x_{0}\right|\right) .
$$

Hence, from (57) and the third equation of [21), the sequence $\left(\mathbf{z}^{n}, x_{s}^{n}\right)$ is bounded in $C^{0}\left([0, T] ; H^{2}\left(\left(0, x_{0}\right) ; \mathbb{R}^{2}\right)\right) \times$ $C^{1}([0, T] ; \mathbb{R})$. By [40, Corollary 4], we can extract a subsequence, which we still denote by $\left(\mathbf{z}^{n}, x_{s}^{n}\right)$ that converges to $\left(\mathbf{u}, y_{s}\right)$ in $\left(C^{0}\left([0, T] ; C^{1}\left(\left[0, x_{0}\right] ; R^{2}\right)\right) \cap C^{1}\left([0, T] ; C^{0}\left(\left[0, x_{0}\right] ; R^{2}\right)\right)\right) \times C^{1}([0, T] ; \mathbb{R})$, which is a classical solution of (21)-23. If we define

$$
J(\mathbf{u})=\left\{\begin{array}{l}
+\infty, \text { if } \mathbf{u} \notin L^{\infty}\left((0, T) ; H^{2}\left(\left(0, x_{0}\right) ; \mathbb{R}^{2}\right)\right), \\
|\mathbf{u}|_{L^{\infty}\left((0, T) ; H^{2}\left(\left(0, x_{0}\right) ; \mathbb{R}^{2}\right)\right.}, \quad \text { if } \mathbf{u} \in L^{\infty}\left((0, T) ; H^{2}\left(\left(0, x_{0}\right) ; \mathbb{R}^{2}\right)\right),
\end{array}\right.
$$

then $J$ is lower semi-continuous and we have

$$
J(\mathbf{u}) \leqslant \varliminf_{n \rightarrow+\infty}\left|\mathbf{z}^{n}\right|_{C^{0}\left([0, T] ; H^{2}\left(\left(0, x_{0}\right) ; \mathbb{R}^{2}\right)\right)}
$$

thus from 58 and the convergence of $\left(\mathbf{z}^{0 n}, x_{s 0}^{n}\right)$ in $H^{2}\left(\left(0, x_{0}\right) ; \mathbb{R}^{2}\right) \times \mathbb{R}$, we have $J(\mathbf{u}) \in \mathbb{R}$ and $\mathbf{u} \in L^{\infty}\left((0, T) ; H^{2}\left(\left(0, x_{0}\right) ; \mathbb{R}^{2}\right)\right)$. Moreover, as $\left(\mathbf{u}, y_{s}\right)$ is a solution to 21$]-23$, we get the extra regularity $\mathbf{u} \in C^{0}\left([0, T] ; H^{2}\left(\left(0, x_{0}\right) ; \mathbb{R}^{2}\right)\right)$. Hence, from the uniqueness of the solution given by 
Lemma 3.1, $\mathbf{u}=\mathbf{z}$ and consequently $y_{s}=x_{s}$, which implies that $\left(\mathbf{z}^{n}, x_{s}^{n}\right)$ converges to $\left(\mathbf{z}, x_{s}\right)$ in $\left(C^{0}\left([0, T] ; C^{1}\left(\left[0, x_{0}\right] ; \mathbb{R}^{2}\right)\right) \cap C^{1}\left([0, T] ; C^{0}\left(\left[0, x_{0}\right] ; \mathbb{R}^{2}\right)\right)\right) \times C^{1}([0, T] ; \mathbb{R})$. Now, we define $V^{n}(t):=$ $V\left(\mathbf{z}^{n}(t, \cdot), x_{s}^{n}(t)\right)$. Note that $V(t)=V\left(\mathbf{z}(t, \cdot), x_{s}(t)\right)$ is continuous with time $t$ and well-defined as, from Lemma 3.1 $\mathbf{z} \in C^{0}\left([0, T] ; H^{2}\left(\left(0, x_{0}\right) ; \mathbb{R}^{2}\right)\right)$. As $\left(\mathbf{z}^{n}, x_{s}^{n}\right)$ belongs to $C^{0}\left([0, T] ; H^{4}\left(\left(0, x_{0}\right) ; \mathbb{R}^{2}\right)\right) \times$ $C^{4}([0, T] ; \mathbb{R})$ and is thus $C^{3}$, and as it is a solution of (21)-(22) with initial condition satisfying (57), we have from $(56)$

$$
\frac{d V^{n}}{d t} \leqslant-\frac{\gamma}{2} V^{n}
$$

thus $V^{n}$ is decreasing on $[0, T]$. Therefore

$$
V^{n}(t)-V^{n}(0) \leqslant-\frac{\gamma t}{2} V^{n}(t), \forall t \in[0, T],
$$

which implies that

$$
\left(1+\frac{\gamma t}{2}\right) V^{n}(t) \leqslant V^{n}(0), \forall t \in[0, T] .
$$

Using the lower semi-continuity of $J$, by the continuity of $V$ and the convergence of $\left(\mathbf{z}^{0 n}, x_{s 0}^{n}\right)$ in $H^{2}\left(\left(0, x_{0}\right) ; \mathbb{R}^{2}\right) \times \mathbb{R}$, we have

$$
\left(1+\frac{\gamma t}{2}\right) V(t) \leqslant V(0), \forall t \in[0, T] .
$$

Note that instead of approximating $\left(\mathbf{z}^{0}, x_{s 0}\right)$, we could have approximated $\left(\mathbf{z}(s, \cdot), x_{s}(s)\right)$ where $s \in$ $[0, T)$ and follow the same procedure as above. Therefore we have in fact for any $s \in[0, T)$

$$
\left(1+\frac{\gamma(t-s)}{2}\right) V(t) \leqslant V(s), \forall t \in[s, T]
$$

thus for any $0 \leqslant s<t \leqslant T$

$$
\frac{V(t)-V(s)}{t-s} \leqslant-\frac{\gamma}{2} V(t),
$$

which implies that (56) holds in the distribution sense. This ends the proof of Lemma 4.2

We now state our final lemma, which gives a sufficient condition so that $V$ defined by (31) -37) satisfies a useful estimate along any $C^{3}$ solutions.

Lemma 4.3. Let $V$ be defined by (31)-(37). If the matrix $\mathbf{A}$ defined by (87)-(92) is positive definite, then for any $T>0$, there exists $\delta_{1}(T)>0$ such that for any $\left.\left(\mathbf{z}, x_{s}\right) \in C^{3}\left([0, T] \times\left[0, x_{0}\right] ; \mathbb{R}^{2}\right)\right) \times$ $C^{3}([0, T] ; \mathbb{R})$ solution of 211 - 23atisfying $\left|\mathbf{z}^{0}\right|_{H^{2}\left(\left(0, x_{0}\right) ; \mathbb{R}^{2}\right)} \leqslant \delta_{1}(T)$ and $\left|x_{s 0}-x_{0}\right| \leqslant \delta_{1}(T)$,

$$
\frac{d V\left(\mathbf{z}(t, \cdot), x_{s}(t)\right)}{d t} \leqslant-\frac{\mu}{2} V\left(\mathbf{z}(t, \cdot), x_{s}(t)\right)+O\left(\left(|\mathbf{z}(t, \cdot)|_{H^{2}\left(\left(0, x_{0}\right) ; \mathbb{R}^{2}\right)}+\left|x_{s}-x_{0}\right|\right)^{3}\right), \quad \forall t \in[0, T] .
$$

Here and hereafter, $O(s)$ means that there exist $\varepsilon>0$ and $C_{1}>0$, both independent of $\mathbf{z}, x_{s}, T$ and $t \in[0, T]$, such that

$$
(s \leqslant \varepsilon) \Longrightarrow\left(|O(s)| \leqslant C_{1} s\right) .
$$

To prove this lemma, we differentiate $V$ with respect to time along any $C^{3}$ solutions and perform several estimates on the different components of $V$. For the sake of simplicity, for any $\mathbf{z} \in$ $C^{0}\left([0, T] ; H^{2}\left(\left(0, x_{0}\right) ; \mathbb{R}^{2}\right)\right)$, we denote from now on $|\mathbf{z}(t, \cdot)|_{H^{2}\left(\left(0, x_{0}\right) ; \mathbb{R}^{2}\right)}$ by $|\mathbf{z}|_{H^{2}}$.

Proof of Lemma 4.3. Let $V$ be given by (31)-37) and $T>0$. Let us assume that $\left(\mathbf{z}, x_{s}\right)$ is a $C^{3}$ solution to the system (21)- 23$)$, with initial condition $\left|\mathbf{z}^{0}\right|_{H^{2}\left(\left(0, x_{0}\right) ; \mathbb{R}^{2}\right)} \leqslant \delta_{1}(T)$ and $\left|x_{s 0}-x_{0}\right| \leqslant \delta_{1}(T)$ respectively with $\delta_{1}(T)>0$ to be chosen later on. Let us examine the different components of the Lyapunov function. We start by studying $V_{1}, V_{2}$ and $V_{3}$ which can be treated similarly as in 3 , Section 4.4]. Differentiating $V_{1}$ along the solution $\left(\mathbf{z}, x_{s}\right)$ and integrating by parts, noticing (38), we have

$$
\begin{aligned}
& \frac{d V_{1}}{d t}=-2 \int_{0}^{x_{0}}\left(p_{1} e^{\frac{-\mu x}{\eta_{1}}} z_{1}\left(1+z_{1}-x \frac{\dot{x}_{s}}{x_{0}}\right) \frac{x_{0}}{x_{s}} z_{1 x}+p_{2} e^{\frac{-\mu x}{\eta_{2}}} z_{2}\left(1-z_{2}+x \frac{\dot{x}_{s}}{x_{0}}\right) \frac{x_{0}}{L-x_{s}} z_{2 x}\right) d x \\
& =-\mu V_{1}-\left[p_{1} e^{\frac{-\mu x}{\eta_{1}}} \frac{x_{0}}{x_{s}} z_{1}^{2}+p_{2} e^{\frac{-\mu x}{\eta_{2}}} \frac{x_{0}}{L-x_{s}} z_{2}^{2}\right]_{0}^{x_{0}}+O\left(\left(|\mathbf{z}|_{H^{2}}+\left|x_{s}-x_{0}\right|\right)^{3}\right) .
\end{aligned}
$$


From 21, we have

$$
\begin{aligned}
& z_{1 t t}+\left(1+z_{1}-x \frac{\dot{x}_{s}}{x_{0}}\right) z_{1 t x} \frac{x_{0}}{x_{s}}+\left(z_{1 t}-x \frac{\ddot{x}_{s}}{x_{0}}\right) z_{1 x} \frac{x_{0}}{x_{s}}+z_{1 t} \frac{\dot{x}_{s}}{x_{s}}=0, \\
& z_{2 t t}+\left(1-z_{2}+x \frac{\dot{x}_{s}}{x_{0}}\right) z_{2 t x} \frac{x_{0}}{L-x_{s}}-\left(z_{2 t}-x \frac{\ddot{x}_{s}}{x_{0}}\right) z_{2 x} \frac{x_{0}}{L-x_{s}}-z_{2 t} \frac{\dot{x}_{s}}{L-x_{s}}=0 .
\end{aligned}
$$

Therefore, similarly to $(68)$, we can obtain

$$
\frac{d V_{2}}{d t}=-\mu V_{2}-\left[p_{1} e^{\frac{-\mu x}{\eta_{1}}} \frac{x_{0}}{x_{s}} z_{1 t}^{2}+p_{2} e^{\frac{-\mu x}{\eta_{2}}} \frac{x_{0}}{L-x_{s}} z_{2 t}^{2}\right]_{0}^{x_{0}}+O\left(\left(|\mathbf{z}|_{H^{2}}+\left|x_{s}-x_{0}\right|\right)^{3}\right) .
$$

From 69 and using 21, we get

$$
\begin{aligned}
& z_{1 t t t}+\left(1+z_{1}-x \frac{\dot{x}_{s}}{x_{0}}\right) z_{1 t t x} \frac{x_{0}}{x_{s}}+2\left(z_{1 t}-x \frac{\ddot{x}_{s}}{x_{0}}\right) z_{1 t x} \frac{x_{0}}{x_{s}}+\frac{\dot{x}_{s}}{x_{s}}\left(z_{1 t t}+z_{1 t} \frac{\dot{x}_{s}}{x_{s}}\right) \\
& +\left(z_{1 t t}-x \frac{\dddot{x}_{s}}{x_{0}}\right) z_{1 x} \frac{x_{0}}{x_{s}}+z_{1 t t} \frac{\dot{x}_{s}}{x_{s}}+z_{1 t} \frac{\ddot{x}_{s} x_{s}-\left(\dot{x}_{s}\right)^{2}}{x_{c}^{2}}=0 \\
& z_{2 t t t}+\left(1-z_{2}+x \frac{\dot{x}_{s}}{x_{0}}\right) z_{2 t t x} \frac{x_{0}}{L-x_{s}}-2\left(z_{2 t}-x \frac{\ddot{x}_{s}}{x_{0}}\right) z_{2 t x} \frac{x_{0}}{L-x_{s}}+\frac{\dot{x}_{s}}{L-x_{s}}\left(-z_{2 t t}+z_{2 t} \frac{\dot{x}_{s}}{L-x_{s}}\right) \\
& -\left(z_{2 t t}-x \frac{\dddot{x}_{s}}{x_{0}}\right) z_{2 x} \frac{x_{0}}{L-x_{s}}-z_{2 t t} \frac{\dot{x}_{s}}{L-x_{s}}-z_{2 t} \frac{\ddot{x}_{s}\left(L-x_{s}\right)+\left(\dot{x}_{s}\right)^{2}}{\left(L-x_{s}\right)^{2}}=0 .
\end{aligned}
$$

Then differentiating $V_{3}$ along the system solutions and using 71 , we have

$$
\begin{aligned}
\frac{d V_{3}}{d t} & \leqslant-\left[p_{1} e^{-\frac{\mu x}{\eta_{1}}} \frac{x_{0}}{x_{s}}\left(z_{1 t t}^{2}\right)\left(1+z_{1}-x \frac{\dot{x}_{s}}{x_{0}}\right)\right]_{0}^{x_{0}}-\left[p_{2} e^{-\frac{\mu x}{\eta_{2}}} \frac{x_{0}}{L-x_{s}} z_{2 t t}^{2}\left(1-z_{2}+x \frac{\dot{x}_{s}}{x_{0}}\right)\right]_{0}^{x_{0}} \\
& -\mu \min \left(\frac{x_{0}}{x_{s}}, \frac{L-x_{0}}{L-x_{s}}\right) V_{3}-\mu \int_{0}^{x_{0}}\left(\frac{x_{0}}{x_{s}} p_{1} e^{-\frac{\mu x}{\eta_{1}}} z_{1 t t}^{2} z_{1}-\frac{L-x_{0}}{L-x_{s}} p_{2} e^{-\frac{\mu x}{\eta_{2}}} z_{2 t t}^{2} z_{2}\right) d x \\
& +\mu \int_{0}^{x_{0}}\left(\frac{x_{0}}{x_{s}} p_{1} e^{-\frac{\mu x}{\eta_{2}}} x z_{1 t t}^{2} \frac{\dot{x}_{s}}{x_{0}}-\frac{L-x_{0}}{L-x_{s}} p_{2} e^{-\frac{\mu x}{\eta_{1}}} x z_{2 t t}^{2} \frac{\dot{x}_{s}}{x_{0}}\right) d x \\
& -3 \int_{0}^{x_{0}}\left(p_{1} e^{-\frac{\mu x}{\eta_{1}}} z_{1 t t}^{2} \frac{\dot{x}_{s}}{x_{s}}-p_{2} e^{-\frac{\mu x}{\eta_{2}}} z_{2 t t}^{2} \frac{\dot{x}_{s}}{L-x_{s}}\right) d x \\
& -\int_{0}^{x_{0}}\left(p_{1} e^{-\frac{\mu x}{\eta_{1}}} z_{1 t t}^{2} z_{1 x} \frac{x_{0}}{x_{s}}-p_{2} e^{-\frac{\mu x}{\eta_{2}}} z_{2 t t}^{2} z_{2 x} \frac{x_{0}}{L-x_{s}}\right) d x \\
& -4 \int_{0}^{x_{0}}\left(p_{1} e^{-\frac{\mu x}{\eta_{1}}} z_{1 t t}\left(z_{1 t}-x \frac{\ddot{x}_{s}}{x_{0}}\right) z_{1 t x} \frac{x_{0}}{x_{s}}-p_{2} e^{-\frac{\mu x}{\eta_{2}}} z_{2 t t}\left(z_{2 t}-x \frac{x_{0}}{x_{0}}\right) z_{2 t x} \frac{\dot{x}_{s}}{L-x_{s}}\right) d x \\
& -2 \int_{0}^{x_{0}}\left(p_{1} e^{-\frac{\mu x}{\eta_{1}}} z_{1 t t}\left(z_{1 t t}+z_{1 t} \frac{\dot{x}_{s}}{x_{s}}\right) \frac{\dot{x}_{s}}{x_{s}}-p_{2} e^{-\frac{\mu x}{\eta_{2}}} z_{2 t t}\left(z_{2 t t}-z_{2 t} \frac{\dot{x}_{s}-x_{s}}{L-x_{s}}\right) d x\right. \\
& -2 \int_{0}^{x_{0}}\left(p_{1} e^{-\frac{\mu x}{\eta_{1}}} z_{1 t t} z_{1 t} \frac{\ddot{x}_{s} x_{s}-\left(\dot{x}_{s}\right)^{2}}{x_{c}^{2}}-p_{2} e^{-\frac{\mu x}{\eta_{2}}} z_{2 t t} z_{2 t} \frac{\ddot{x}_{s}\left(L-x_{s}\right)+\left(\dot{x}_{s}\right)^{2}}{\left(L-x_{s}\right)^{2}} d x\right. \\
& -2 \int_{0}^{x_{0}}\left(p_{1} e^{-\frac{\mu x}{\eta_{1}}} z_{1 t t}\left(z_{1 t t}-x \frac{\dddot{x}_{s}}{x_{0}}\right) z_{1 x} \frac{x_{0}}{x_{s}}-p_{2} e^{-\frac{\mu x}{\eta_{2}}} z_{2 t t}\left(z_{2 t t}-x \frac{x_{0}}{x_{0}}\right) z_{2 x} \frac{x_{L}-x_{s}}{L}\right) d x .
\end{aligned}
$$

Observe that, while previously all the cubic terms in $\mathbf{z}$ could be bounded by $|\mathbf{z}|_{H^{2}}^{3}$, here in the last line in $(72)$ we have $\dddot{x}_{s}$ which is proportional to $\mathbf{z}_{t t}\left(t, x_{0}\right)$ and cannot be roughly bounded by the $|\mathbf{z}|_{H^{2}}$ norm. To overcome this difficulty, we transform these terms using Young's inequality and we get

$$
\begin{aligned}
2 \int_{0}^{x_{0}} & \left(p_{1} e^{-\frac{\mu x}{\eta_{1}}} z_{1 t t}\left(x \frac{\dddot{x}_{s}}{x_{0}}\right) z_{1 x} \frac{x_{0}}{x_{s}}-p_{2} e^{-\frac{\mu x}{\eta_{2}}} z_{2 t t}\left(x \frac{\dddot{x}_{s}}{x_{0}}\right) z_{2 x} \frac{x_{0}}{L-x_{s}}\right) d x \\
& \leqslant C|\mathbf{z}(t, \cdot)|_{C^{1}\left(\left[0, x_{0}\right] ; \mathbb{R}^{2}\right)}\left(z_{1 t t}\left(t, x_{0}\right)+z_{2 t t}\left(t, x_{0}\right)\right)^{2}+O\left(|\mathbf{z}(t, \cdot)|_{C^{1}\left(\left[0, x_{0}\right] ; \mathbb{R}^{2}\right)}|\mathbf{z}|_{H^{2}}^{2}\right),
\end{aligned}
$$

where $C$ denotes a constant, independent of $\mathbf{z}, x_{s}, T$ and $t \in[0, T]$. Note that the first term on the right is now proportional to $\mathbf{z}_{t t}^{2}\left(t, x_{0}\right)$ with a proportionality coefficient $C|\mathbf{z}(t, \cdot)|_{C^{1}\left(\left[0, x_{0}\right] ; \mathbb{R}^{2}\right)}$ that, by 
Sobolev inequality, can be made sufficiently small provided that $|\mathbf{z}|_{H^{2}}$ is sufficiently small and thus can be dominated by the boundary terms. More precisely, from $\sqrt{72}$ and $(73)$ we have

$$
\begin{aligned}
\frac{d V_{3}}{d t} \leqslant & -\mu V_{3}-\left[p_{1} e^{\frac{-\mu x}{\eta_{1}}} \frac{x_{0}}{x_{s}}\left(z_{1 t t}^{2}\right)\right]_{0}^{x_{0}}-\left[p_{2} e^{\frac{-\mu x}{\eta_{2}}} \frac{x_{0}}{L-x_{s}} z_{2 t t}^{2}\right]_{0}^{x_{0}} \\
& +O\left(|\mathbf{z}|_{H^{2}}\right)\left(z_{1 t t}^{2}\left(t, x_{0}\right)+z_{2 t t}^{2}\left(t, x_{0}\right)\right)+O\left(\left(|\mathbf{z}|_{H^{2}}+\left|x_{s}-x_{0}\right|\right)^{3}\right) .
\end{aligned}
$$

Let us now deal with the term $V_{4}$ that takes into account the position of the jump. In the following, we use notations $\mathbf{z}(0)$ and $\mathbf{z}\left(x_{0}\right)$ instead of $\mathbf{z}(t, 0)$ and $\mathbf{z}\left(t, x_{0}\right)$ for simplicity. We have

$$
\begin{aligned}
\frac{d V_{4}}{d t}= & -\int_{0}^{x_{0}} \bar{p}_{1} e^{\frac{-\mu x}{\eta_{1}}}\left(1+z_{1}-x \frac{\dot{x}_{s}}{x_{0}}\right) z_{1 x}\left(x_{s}-x_{0}\right) \frac{x_{0}}{x_{s}} d x+\int_{0}^{x_{0}} \bar{p}_{1} e^{\frac{-\mu x}{\eta_{1}}} z_{1} \dot{x}_{s} d x \\
& -\int_{0}^{x_{0}} \bar{p}_{2} e^{\frac{-\mu x}{\eta_{2}}}\left(1-z_{2}+x \frac{\dot{x}_{s}}{x_{0}}\right) z_{2 x}\left(x_{s}-x_{0}\right) \frac{x_{0}}{L-x_{s}} d x \\
& +\int_{0}^{x_{0}} \bar{p}_{2} e^{\frac{-\mu x}{\eta_{2}}} z_{2} \dot{x}_{s} d x+2 \kappa \dot{x}_{s}\left(x_{s}-x_{0}\right) \\
= & -\left(x_{s}-x_{0}\right)\left[\bar{p}_{1} e^{\frac{-\mu x}{\eta_{1}}} \frac{x_{0}}{x_{s}} z_{1}+\bar{p}_{2} e^{\frac{-\mu x}{\eta_{2}}} \frac{x_{0}}{L-x_{s}} z_{2}\right]_{0}^{x_{0}}-\mu\left(V_{4}-\kappa\left(x_{s}-x_{0}\right)^{2}\right) \\
& +\frac{z_{1}\left(x_{0}\right)+z_{2}\left(x_{0}\right)}{2}\left(\int_{0}^{x_{0}} \bar{p}_{1} e^{-\frac{\mu x}{\eta_{1}}} z_{1} d x\right)+\frac{z_{1}\left(x_{0}\right)+z_{2}\left(x_{0}\right)}{2}\left(\int_{0}^{x_{0}} \bar{p}_{2} e^{-\frac{\mu x}{\eta_{2}}} z_{2} d x\right) \\
& +\kappa\left(z_{1}\left(x_{0}\right)+z_{2}\left(x_{0}\right)\right)\left(x_{s}-x_{0}\right)+O\left(\left(|\mathbf{z}|_{H^{2}}+\left|x_{s}-x_{0}\right|\right)^{3}\right) .
\end{aligned}
$$

According to Young's inequality, for any positive $\varepsilon_{1}$ and $\varepsilon_{2}$, we have

$$
\begin{aligned}
& \frac{z_{1}\left(x_{0}\right)+z_{2}\left(x_{0}\right)}{2}\left(\int_{0}^{x_{0}} \bar{p}_{1} e^{-\frac{\mu x}{\eta_{1}}} z_{1} d x\right) \leqslant \frac{\varepsilon_{1}}{4}\left(\frac{z_{1}\left(x_{0}\right)+z_{2}\left(x_{0}\right)}{2}\right)^{2}+\frac{1}{\varepsilon_{1}}\left(\int_{0}^{x_{0}} \bar{p}_{1} e^{-\frac{\mu x}{\eta_{1}}} z_{1} d x\right)^{2}, \\
& \frac{z_{1}\left(x_{0}\right)+z_{2}\left(x_{0}\right)}{2}\left(\int_{0}^{x_{0}} \bar{p}_{2} e^{-\frac{\mu x}{\eta_{2}}} z_{2} d x\right) \leqslant \frac{\varepsilon_{2}}{4}\left(\frac{z_{1}\left(x_{0}\right)+z_{2}\left(x_{0}\right)}{2}\right)^{2}+\frac{1}{\varepsilon_{2}}\left(\int_{0}^{x_{0}} \bar{p}_{2} e^{-\frac{\mu x}{\eta_{2}}} z_{2} d x\right)^{2} .
\end{aligned}
$$

Then using the boundary condition $(22)$ and Cauchy-Schwarz inequality, 75 becomes

$$
\begin{aligned}
\frac{d V_{4}}{d t} \leqslant & -\mu V_{4}-\bar{p}_{1}\left(x_{s}-x_{0}\right) \frac{x_{0}}{x_{s}}\left(\left(e^{-\frac{\mu x_{0}}{\eta_{1}}}-k_{1}\right) z_{1}\left(x_{0}\right)+b_{1}\left(x_{s}-x_{0}\right)\right) \\
& -\bar{p}_{2}\left(x_{s}-x_{0}\right) \frac{x_{0}}{L-x_{s}}\left(\left(e^{-\frac{\mu x_{0}}{\eta_{2}}}-k_{2}\right) z_{2}\left(x_{0}\right)+b_{2}\left(x_{s}-x_{0}\right)\right) \\
& +\left(\varepsilon_{1}+\varepsilon_{2}\right) \frac{z_{1}^{2}\left(x_{0}\right)+z_{2}^{2}\left(x_{0}\right)}{8}+\max \left\{\frac{\Theta_{1}}{\varepsilon_{1}}, \frac{\Theta_{2}}{\varepsilon_{2}}\right\} V_{1} \\
& +\kappa\left(x_{s}-x_{0}\right)\left(z_{1}\left(x_{0}\right)+z_{2}\left(x_{0}\right)\right)+\mu \kappa\left(x_{s}-x_{0}\right)^{2}+O\left(\left(|\mathbf{z}|_{H^{2}}+\left|x_{s}-x_{0}\right|\right)^{3}\right) .
\end{aligned}
$$

Let us now consider $V_{5}$. From $(36)$ and $(69)$, one has similarly

$$
\begin{aligned}
\frac{d V_{5}}{d t}= & -\int_{0}^{x_{0}} \bar{p}_{1} e^{\frac{-\mu x}{\eta_{1}}} z_{1 t x} \dot{x}_{s} \frac{x_{0}}{x_{s}} d x+\int_{0}^{x_{0}} \bar{p}_{1} e^{\frac{-\mu x}{\eta_{1}}} z_{1 t} \ddot{x}_{s} d x \\
& -\int_{0}^{x_{0}} \bar{p}_{2} e^{\frac{-\mu x}{\eta_{2}}} z_{2 t x} \dot{x}_{s} \frac{x_{0}}{L-x_{s}} d x+\int_{0}^{x_{0}} \bar{p}_{2} e^{\frac{-\mu x}{\eta_{2}}} z_{2 t} \ddot{x}_{s} d x+2 \kappa \ddot{x}_{s} \dot{x}_{s}+O\left(\left(|\mathbf{z}|_{H^{2}}+\left|x_{s}-x_{0}\right|\right)^{3}\right) \\
= & -\dot{x}_{s}\left[\bar{p}_{1} e^{\frac{-\mu x}{\eta_{1}}} \frac{x_{0}}{x_{s}} z_{1 t}+\bar{p}_{2} e^{\frac{-\mu x}{\eta_{2}}} \frac{x_{0}}{L-x_{s}} z_{2 t}\right]_{0}^{x_{0}}-\mu\left(V_{5}-\kappa\left(\dot{x}_{s}\right)^{2}\right) \\
& +\frac{z_{1 t}\left(x_{0}\right)+z_{2 t}\left(x_{0}\right)}{2}\left(\int_{0}^{x_{0}} \bar{p}_{1} e^{-\frac{\mu x}{\eta_{1}}} z_{1 t} d x\right)+\frac{z_{1 t}\left(x_{0}\right)+z_{2 t}\left(x_{0}\right)}{2}\left(\int_{0}^{x_{0}} \bar{p}_{2} e^{-\frac{\mu x}{\eta_{2}}} z_{2 t} d x\right) \\
& +\kappa\left(z_{1 t}\left(x_{0}\right)+z_{2 t}\left(x_{0}\right)\right) \dot{x}_{s}+O\left(\left(|\mathbf{z}|_{H^{2}}+\left|x_{s}-x_{0}\right|\right)^{3}\right) .
\end{aligned}
$$

By differentiating 22 with respect to time, we have

$$
\begin{aligned}
& z_{1 t}(0)=k_{1} z_{1 t}\left(x_{0}\right)-b_{1} \dot{x}_{s}, \\
& z_{2 t}(0)=k_{2} z_{2 t}\left(x_{0}\right)-b_{2} \dot{x}_{s},
\end{aligned}
$$


and therefore using Cauchy-Schwarz and Young's inequalities, we get

$$
\begin{aligned}
\frac{d V_{5}}{d t} \leqslant & -\mu V_{5}-\bar{p}_{1} \dot{x}_{s} \frac{x_{0}}{x_{s}}\left(\left(e^{-\frac{\mu x_{0}}{\eta_{1}}}-k_{1}\right) z_{1 t}\left(x_{0}\right)+b_{1} \dot{x}_{s}\right) \\
& -\bar{p}_{2} \dot{x}_{s} \frac{x_{0}}{L-x_{s}}\left(\left(e^{-\frac{\mu x_{0}}{\eta_{2}}}-k_{2}\right) z_{2 t}\left(x_{0}\right)+b_{2} \dot{x}_{s}\right) \\
& +\left(\varepsilon_{1}+\varepsilon_{2}\right) \frac{z_{1 t}^{2}\left(x_{0}\right)+z_{2 t}^{2}\left(x_{0}\right)}{8}+\max \left\{\frac{\Theta_{1}}{\varepsilon_{1}}, \frac{\Theta_{2}}{\varepsilon_{2}}\right\} V_{2} \\
& +\kappa \dot{x}_{s}\left(z_{1 t}\left(x_{0}\right)+z_{2 t}\left(x_{0}\right)\right)+\mu \kappa\left(\dot{x}_{s}\right)^{2}+O\left(\left(|\mathbf{z}|_{H^{2}}+\left|x_{s}-x_{0}\right|\right)^{3}\right) .
\end{aligned}
$$

Furthermore, by differentiating $(78)$ with respect to time, we have

$$
\begin{aligned}
& z_{1 t t}(0)=k_{1} z_{1 t t}\left(x_{0}\right)-b_{1} \ddot{x}_{s}, \\
& z_{2 t t}(0)=k_{2} z_{2 t t}\left(x_{0}\right)-b_{2} \ddot{x}_{s},
\end{aligned}
$$

and therefore using also 71 , one has

$$
\begin{aligned}
\frac{d V_{6}}{d t}= & -\int_{0}^{x_{0}} \bar{p}_{1} e^{\frac{-\mu x}{\eta_{1}}} z_{1 t t x} \ddot{x}_{s} \frac{x_{0}}{x_{s}} d x+\int_{0}^{x_{0}} \bar{p}_{1} e^{\frac{-\mu x}{\eta_{1}}} z_{1 t t} \dddot{x}_{s} d x \\
& -\int_{0}^{x_{0}} \bar{p}_{2} e^{\frac{-\mu x}{\eta_{2}}} z_{2 t t x} \ddot{x}_{s} \frac{x_{0}}{L-x_{s}} d x+\int_{0}^{x_{0}} \bar{p}_{2} e^{\frac{-\mu x}{\eta_{2}}} z_{2 t t} \dddot{x}_{s} d x+2 \kappa \dddot{x}_{s} \ddot{x}_{s}+\int_{0}^{x_{0}} \bar{p}_{1} e^{\frac{-\mu x}{\eta_{1}}} \ddot{x}_{s}\left(x \frac{\dddot{x}_{s}}{x_{0}}\right) z_{1 x} \frac{x_{0}}{x_{s}} d x \\
& -\int_{0}^{x_{0}} \bar{p}_{2} e^{\frac{-\mu x}{\eta_{2}}} \ddot{x}_{s}\left(x \frac{\dddot{x}_{s}}{x_{0}}\right) z_{2 x} \frac{x_{0}}{L-x_{s}} d x+O\left(\left(|\mathbf{z}|_{H^{2}}+\left|x_{s}-x_{0}\right|\right)^{3}\right) \\
= & -\ddot{x}_{s}\left[\bar{p}_{1} e^{\frac{-\mu x}{\eta_{1}}} \frac{x_{0}}{x_{s}} z_{1 t t}+\bar{p}_{2} e^{\frac{-\mu x}{\eta_{2}}} \frac{x_{0}}{L-x_{s}} z_{2 t t}\right]_{0}^{x_{0}}-\mu\left(V_{6}-\kappa\left(\ddot{x}_{s}\right)^{2}\right) \\
& +\frac{z_{1 t t}\left(x_{0}\right)+z_{2 t t}\left(x_{0}\right)}{2}\left(\int_{0}^{x_{0}} \bar{p}_{1} e^{-\frac{\mu x}{\eta_{1}}} z_{1 t t} d x\right)+\frac{z_{1 t t}\left(x_{0}\right)+z_{2 t t}\left(x_{0}\right)}{2}\left(\int_{0}^{x_{0}} \bar{p}_{2} e^{-\frac{\mu x}{\eta_{2}}} z_{2 t t} d x\right) \\
& +\kappa\left(z_{1 t t}\left(x_{0}\right)+z_{2 t t}\left(x_{0}\right)\right) \ddot{x}_{s}+\int_{0}^{x_{0}} \bar{p}_{1} e^{\frac{-\mu x}{\eta_{1}}} \ddot{x}_{s}\left(x \frac{\dddot{x}_{s}}{x_{0}}\right) z_{1 x} \frac{x_{0}}{x_{s}} d x \\
& -\int_{0}^{x_{0}} \bar{p}_{2} e^{\frac{-\mu x}{\eta_{2}}} \ddot{x}_{s}\left(x \frac{\dddot{x}_{s}}{x_{0}}\right) z_{2 x} \frac{x_{0}}{L-x_{s}} d x+O\left(\left(|\mathbf{z}|_{H^{2}}+\left|x_{s}-x_{0}\right|\right)^{3}\right) .
\end{aligned}
$$

Note that, as above for $V_{3}$, here appears again $\dddot{x}_{s}$ which is proportional to $\mathbf{z}_{t t}\left(t, x_{0}\right)$ and cannot be bounded by $|\mathbf{z}|_{H^{2}}$. We therefore use Cauchy-Schwarz and Young's inequalities as previously and the boundary condition 80, to get

$$
\begin{aligned}
\frac{d V_{6}}{d t} \leqslant & -\mu V_{6}-\bar{p}_{1} \ddot{x}_{s} \frac{x_{0}}{x_{s}}\left(\left(e^{-\frac{\mu x_{0}}{\eta_{1}}}-k_{1}\right) z_{1 t t}\left(x_{0}\right)+b_{1} \ddot{x}_{s}\right) \\
& -\bar{p}_{2} \ddot{x}_{s} \frac{x_{0}}{L-x_{s}}\left(\left(e^{-\frac{\mu x_{0}}{\eta_{2}}}-k_{2}\right) z_{2 t t}\left(x_{0}\right)+b_{2} \ddot{x}_{s}\right) \\
& +\left(\varepsilon_{1}+\varepsilon_{2}\right) \frac{z_{1 t t}^{2}\left(x_{0}\right)+z_{2 t t}^{2}\left(x_{0}\right)}{8}+\max \left\{\frac{\Theta_{1}}{\varepsilon_{1}}, \frac{\Theta_{2}}{\varepsilon_{2}}\right\} V_{2} \\
& +\kappa \ddot{x}_{s}\left(z_{1 t t}\left(x_{0}\right)+z_{2 t t}\left(x_{0}\right)\right)+\mu \kappa\left(\ddot{x}_{s}\right)^{2}+O\left(|\mathbf{z}|_{H^{2}}\right)\left(z_{1 t t}^{2}\left(x_{0}\right)+z_{2 t t}^{2}\left(x_{0}\right)\right) \\
& +O\left(\left(|\mathbf{z}|_{H^{2}}+\left|x_{s}-x_{0}\right|\right)^{3}\right) .
\end{aligned}
$$


Hence, from 68, (77) and the boundary conditions 22 , we have

$$
\begin{aligned}
\frac{d V_{1}}{d t}+\frac{d V_{4}}{d t} \leqslant & -\mu\left(V_{1}+V_{4}\right) \\
& +\max \left\{\frac{\Theta_{1}}{\varepsilon_{1}}, \frac{\Theta_{2}}{\varepsilon_{2}}\right\} V_{1} \\
& +\left[\frac{x_{0}}{x_{s}} p_{1}\left(k_{1}^{2}-e^{-\frac{\mu x_{0}}{\eta_{1}}}\right)+\frac{\varepsilon_{1}+\varepsilon_{2}}{8}\right] z_{1}^{2}\left(x_{0}\right) \\
& +\left[\frac{x_{0}}{L-x_{s}} p_{2}\left(k_{2}^{2}-e^{-\frac{\mu x_{0}}{\eta_{2}}}\right)+\frac{\varepsilon_{1}+\varepsilon_{2}}{8}\right] z_{2}^{2}\left(x_{0}\right) \\
& +\left[-2 \frac{x_{0}}{x_{s}} p_{1} b_{1} k_{1}-\frac{x_{0}}{x_{s}} \bar{p}_{1}\left(e^{-\frac{\mu x_{0}}{\eta_{1}}}-k_{1}\right)+\kappa\right] z_{1}\left(x_{0}\right)\left(x_{s}-x_{0}\right) \\
& +\left[-2 \frac{x_{0}}{L-x_{s}} p_{2} b_{2} k_{2}-\frac{x_{0}}{L-x_{s}} \bar{p}_{2}\left(e^{-\frac{\mu x_{0}}{\eta_{2}}}-k_{2}\right)+\kappa\right] z_{2}\left(x_{0}\right)\left(x_{s}-x_{0}\right) \\
& +\left[\frac{x_{0}}{x_{s}} p_{1} b_{1}^{2}+\frac{x_{0}}{L-x_{s}} p_{2} b_{2}^{2}-\frac{x_{0}}{x_{s}} \bar{p}_{1} b_{1}-\frac{x_{0}}{L-x_{s}} \bar{p}_{2} b_{2}+\mu \kappa\right]\left(x_{s}-x_{0}\right)^{2} \\
& +O\left(\left(|\mathbf{z}|_{H^{2}}+\left|x_{s}-x_{0}\right|\right)^{3}\right) .
\end{aligned}
$$

Let us now select $\varepsilon_{1}$ and $\varepsilon_{2}$ as follows:

$$
\varepsilon_{1}=2 \frac{\Theta_{1}}{\mu}, \quad \varepsilon_{2}=2 \frac{\Theta_{2}}{\mu},
$$

where $\Theta_{1}$ and $\Theta_{2}$ are defined in $(45)$. Then 82 can be rewritten in the following compact form:

$$
\frac{d V_{1}}{d t}+\frac{d V_{4}}{d t} \leqslant-\frac{\mu}{2} V_{1}-\mu V_{4}-\mathbf{Z}^{T} \mathbf{A}_{0} \mathbf{Z}+O\left(\left(|\mathbf{z}|_{H^{2}}+\left|x_{s}-x_{0}\right|\right)^{3}\right) .
$$

This expression involves the quadratic form $\mathbf{Z}^{T} \mathbf{A}_{0} \mathbf{Z}$ with the vector $\mathbf{Z}$ defined as

$$
\mathbf{Z}=\left(z_{1}\left(x_{0}\right) z_{2}\left(x_{0}\right)\left(x_{s}-x_{0}\right)\right)^{T} .
$$

and the matrix $\mathbf{A}_{0}$ satisfies

$$
\mathbf{A}_{0}=\mathbf{A}+O\left(\left|x_{s}-x_{0}\right|\right)
$$

where $\mathbf{A}$ is given by

$$
\mathbf{A}=\left(\begin{array}{ccc}
a_{11} & 0 & a_{13} \\
0 & a_{22} & a_{23} \\
a_{31} & a_{32} & a_{33}
\end{array}\right)
$$

with

$$
\begin{aligned}
& a_{11}=p_{1}\left(e^{-\frac{\mu x_{0}}{\eta_{1}}}-k_{1}^{2}\right)-\frac{\varepsilon_{1}+\varepsilon_{2}}{8}, \\
& a_{13}=a_{31}=p_{1} b_{1} k_{1}+\frac{\bar{p}_{1}}{2}\left(e^{-\frac{\mu x_{0}}{\eta_{1}}}-k_{1}\right)-\frac{\kappa}{2}, \\
& a_{22}=\frac{x_{0}}{L-x_{0}} p_{2}\left(e^{-\frac{\mu x_{0}}{\eta_{2}}}-k_{2}^{2}\right)-\frac{\varepsilon_{1}+\varepsilon_{2}}{8}, \\
& a_{23}=a_{32}=\frac{x_{0}}{L-x_{0}} p_{2} b_{2} k_{2}+\frac{x_{0}}{L-x_{0}} \frac{\bar{p}_{2}}{2}\left(e^{-\frac{\mu x_{0}}{\eta_{2}}}-k_{2}\right)-\frac{\kappa}{2}, \\
& a_{33}=-p_{1} b_{1}^{2}-\frac{x_{0}}{L-x_{0}} p_{2} b_{2}^{2}+\bar{p}_{1} b_{1}+\frac{x_{0}}{L-x_{0}} \bar{p}_{2} b_{2}-\mu \kappa .
\end{aligned}
$$

Similarly, from $\sqrt{70}$ and $(79)$, we get

$$
\frac{d V_{2}}{d t}+\frac{d V_{5}}{d t} \leqslant-\frac{\mu}{2} V_{2}-\mu V_{5}-\mathbf{Z}_{t}^{T} \mathbf{A}_{0} \mathbf{Z}_{t}+O\left(\left(|\mathbf{z}|_{H^{2}}+\left|x_{s}-x_{0}\right|\right)^{3}\right),
$$


while from (74) and (81), we have

$$
\frac{d V_{3}}{d t}+\frac{d V_{6}}{d t} \leqslant-\frac{\mu}{2} V_{3}-\mu V_{6}-\mathbf{Z}_{t t}^{T} \mathbf{A}_{1} \mathbf{Z}_{t t}+O\left(\left(|\mathbf{z}|_{H^{2}}+\left|x_{s}-x_{0}\right|\right)^{3}\right)
$$

with

$$
\mathbf{A}_{1}=\mathbf{A}_{0}+\left(\begin{array}{ccc}
O\left(|\mathbf{z}|_{H^{2}}\right) & 0 & 0 \\
0 & O\left(|\mathbf{z}|_{H^{2}}\right) & 0 \\
0 & 0 & 0
\end{array}\right) .
$$

If $\mathbf{A}$ is positive definite, from (86) and (95) and by continuity, $\mathbf{A}_{0}$ and $\mathbf{A}_{1}$ are also positive definite provided that $|\mathbf{z}|_{H^{2}}$ and $\left|x_{s}-x_{0}\right|$ are sufficiently small. Hence, from (84), (93), (94) and Lemma 3.1. there exists $\delta_{1}(T)>0$ such that, if $\left|\mathbf{z}^{0}\right|_{H^{2}\left(\left(0, x_{0}\right) ; \mathbb{R}^{2}\right)} \leqslant \delta_{1}(T)$ and $\left|x_{s 0}-x_{0}\right| \leqslant \delta_{1}(T)$, one has

$$
\frac{d V}{d t} \leqslant-\frac{\mu}{2} V+O\left(\left(|\mathbf{z}|_{H^{2}}+\left|x_{s}-x_{0}\right|\right)^{3}\right),
$$

which ends the proof of Lemma 4.3 .

Let us now prove Theorem 4.1

Proof of Theorem 4.1. From Lemma 4.1 and Lemma 4.2, all it remains to do is to show that for any $\gamma>0$, under conditions (16) there exist $\mu, p_{1}, p_{2}, \bar{p}_{1}$ and $\bar{p}_{2}$ satisfying (44) and such that $V$ given by (31)-(37) decreases exponentially with rate $\gamma / 2$ along any $C^{3}$ solution of the system (21)-(23). Using Lemma 4.3 we first show that for any $\gamma>0$ there exists $\mu>\gamma$, and positive parameters $p_{1}, p_{2}, \bar{p}_{1}$ and $\bar{p}_{2}$ satisfying (44) and such that the matrix $\mathbf{A}$ defined by (87)- 92 is positive definite, which implies that (67) holds. Then, we show that this implies the exponential decay of $V$ with decay rate $\gamma / 2$ along any $C^{3}$ solution of 21)-23.

Let us start by selecting $p_{1}$ and $p_{2}$ as

$$
p_{1}=\frac{\bar{p}_{1}}{2 b_{1}}, \quad p_{2}=\frac{\bar{p}_{2}}{2 b_{2}} .
$$

Then the cross terms 89, ,91) of the matrix $\mathbf{A}$ become

$$
a_{13}=a_{31}=\frac{\bar{p}_{1}}{2} e^{-\frac{\mu x_{0}}{\eta_{1}}}-\frac{\kappa}{2}, \quad a_{23}=a_{32}=\frac{x_{0}}{L-x_{0}} \frac{\bar{p}_{2}}{2} e^{-\frac{\mu x_{0}}{\eta_{2}}}-\frac{\kappa}{2} .
$$

Let $\bar{p}_{1}$ and $\bar{p}_{2}$ be selected as

$$
\bar{p}_{1}=\kappa e^{\frac{\mu x_{0}}{\eta_{1}}}, \quad \bar{p}_{2}=\kappa \frac{L-x_{0}}{x_{0}} e^{\frac{\mu x_{0}}{\eta_{2}}} .
$$

Then we have

$$
a_{13}=a_{31}=0, \quad a_{23}=a_{32}=0
$$

such that $\mathbf{A}$ can now be rewritten as

$$
\mathbf{A}=\left(\begin{array}{ccc}
a_{11} & 0 & 0 \\
0 & a_{22} & 0 \\
0 & 0 & a_{33}
\end{array}\right) .
$$

Moreover from 97] and 997, we get

$$
a_{33}=\frac{\bar{p}_{1}}{2} b_{1}+\frac{x_{0}}{L-x_{0}} \frac{\bar{p}_{2}}{2} b_{2}-\mu \kappa=\frac{\kappa}{2} b_{1} e^{\frac{\mu x_{0}}{\eta_{1}}}+\frac{\kappa}{2} b_{2} e^{\frac{\mu x_{0}}{\eta_{2}}}-\mu \kappa .
$$

As conditions (16) are strict inequalities, by continuity it follows that we can select $\mu>\gamma$ such that these conditions (16) are still satisfied with $\mu$ instead of $\gamma$ such that

$$
\mu e^{-\frac{\mu x_{0}}{\eta_{1}}}<b_{1}<\frac{\mu e^{-\frac{\mu x_{0}}{\eta_{1}}}}{1-e^{-\frac{\mu x_{0}}{\eta_{1}}}}, \quad \mu e^{-\frac{\mu x_{0}}{\eta_{2}}}<b_{2}<\frac{\mu e^{-\frac{\mu x_{0}}{\eta_{2}}}}{1-e^{-\frac{\mu x_{0}}{\eta_{2}}}},
$$

this together with 102 gives

$$
a_{33}>0 .
$$


From (45), (83), 88), (90), 97) and (99), we have

$$
\begin{aligned}
& a_{11}=\frac{\kappa}{2 b_{1}}\left(1-k_{1}^{2} e^{\frac{\mu x_{0}}{\eta_{1}}}\right)-\frac{\kappa}{2 \mu^{2}}\left[b_{1}\left(e^{\frac{\mu x_{0}}{\eta_{1}}}-1\right)+b_{2}\left(e^{\frac{\mu x_{0}}{\eta_{2}}}-1\right)\right], \\
& a_{22}=\frac{\kappa}{2 b_{2}}\left(1-k_{2}^{2} e^{\frac{\mu x_{0}}{\eta_{2}}}\right)-\frac{\kappa}{2 \mu^{2}}\left[b_{1}\left(e^{\frac{\mu x_{0}}{\eta_{1}}}-1\right)+b_{2}\left(e^{\frac{\mu x_{0}}{\eta_{2}}}-1\right)\right] .
\end{aligned}
$$

Then, under assumptions 16 , it can be checked that

$$
a_{11}>0, \quad a_{22}>0
$$

This implies that $\mathbf{A}$ is positive definite.

Thus from Lemma 4.3 , for any $T>0$, there exists $\delta_{1}(T)>0$ such that for any $\left(\mathbf{z}, x_{s}\right) \in C^{3}([0, T] \times$ $\left.\left.\left[0, x_{0}\right] ; \mathbb{R}^{2}\right)\right) \times C^{3}([0, T] ; \mathbb{R})$ solution of $21-23$ satisfying $\left|\mathbf{z}^{0}\right|_{H^{2}\left((0, L) ; \mathbb{R}^{2}\right)} \leqslant \delta_{1}(T)$ and $\left|x_{s 0}-x_{0}\right| \leqslant$ $\delta_{1}(T)$, one has

$$
\frac{d V}{d t} \leqslant-\frac{\mu}{2} V+O\left(\left(|\mathbf{z}|_{H^{2}}+\left|x_{s}-x_{0}\right|\right)^{3}\right)
$$

Now let us remark that from condition 103 we have

$$
\max \left(2 \frac{b_{1} \eta_{1}}{\mu} e^{\frac{\mu x_{0}}{\eta_{1}}}\left(1-e^{-\frac{\mu x_{0}}{\eta_{1}}}\right), 2 \frac{L-x_{0}}{x_{0}} \frac{b_{2} \eta_{2}}{\mu} e^{\frac{\mu x_{0}}{\eta_{2}}}\left(1-e^{-\frac{\mu x_{0}}{\eta_{2}}}\right)\right)<2 .
$$

Therefore, there exists $\kappa>1$ such that

$$
\max \left(2 \kappa \frac{b_{1} \eta_{1}}{\mu} e^{\frac{\mu x_{0}}{\eta_{1}}}\left(1-e^{-\frac{\mu x_{0}}{\eta_{1}}}\right), 2 \kappa \frac{L-x_{0}}{x_{0}} \frac{b_{2} \eta_{2}}{\mu} e^{\frac{\mu x_{0}}{\eta_{2}}}\left(1-e^{-\frac{\mu x_{0}}{\eta_{2}}}\right)\right)<2,
$$

which means from $(97)$ and $(99)$ that 44 is satisfied. Hence from 108 and Lemma 4.1, since $\mu>\gamma$, there exists $\delta_{0}(T) \leqslant \delta_{1}(T)$ such that, if $\left|\mathbf{z}^{0}\right|_{H^{2}\left(\left(0, x_{0}\right) ; \mathbb{R}^{2}\right)} \leqslant \delta_{0}(T)$ and $\left|x_{s 0}-x_{0}\right| \leqslant \delta_{0}(T)$, then

$$
\frac{d V}{d t} \leqslant-\frac{\gamma}{2} V
$$

along the $C^{3}$ solutions of the system 21)-(23). Thus from Lemma 4.2 (111) holds along the $C^{0}\left([0, T] ; H^{2}\left(\left(0, x_{0}\right) ; \mathbb{R}^{2}\right)\right) \times C^{1}([0, T] ; \mathbb{R})$ solutions of $(21)-(23)$ in a distribution sense.

So far $\delta_{0}(T)$ may depend on $T$, while $\delta^{*}$ in Theorem 4.1 does not depend on $T$. The only thing left to check is that we can find $\delta^{*}$ independent of $T$ such that if $\left|\mathbf{z}^{0}\right|_{H^{2}\left(\left(0, x_{0}\right) ; \mathbb{R}^{2}\right)} \leqslant \delta^{*}$ and $\left|x_{s 0}-x_{0}\right| \leqslant \delta^{*}$, then (111) holds on $(0, T)$ for any $T>0$. As the constant $\beta$ involved in Lemma 4.1 does not depend on $T$, there exists $T_{1}>0$ such that

$$
\beta^{-2} e^{-\frac{\gamma}{2} T_{1}}<\frac{1}{2}
$$

As $T_{1} \in(0,+\infty)$, from Lemma 3.1 , we can choose $\delta_{0}\left(T_{1}\right)>0$ satisfying $C\left(T_{1}\right) \delta_{0}\left(T_{1}\right)<\beta / 2$, such that for every $x_{s 0} \in(0, L)$ and $\mathbf{z}^{0} \in H^{2}\left(\left(0, x_{0}\right) ; \mathbb{R}^{2}\right)$ satisfying the compatibility conditions 25$]-26$ and

$$
\left|\mathbf{z}^{0}\right|_{H^{2}\left(\left(0, x_{0}\right) ; \mathbb{R}^{2}\right)} \leqslant \delta_{0}\left(T_{1}\right), \quad\left|x_{s 0}-x_{0}\right| \leqslant \delta_{0}\left(T_{1}\right),
$$

there exists a unique solution $\left(\mathbf{z}, x_{s}\right) \in C^{0}\left(\left[0, T_{1}\right] ; H^{2}\left(\left(0, x_{0}\right) ; \mathbb{R}^{2}\right)\right) \times C^{1}\left(\left[0, T_{1}\right] ; \mathbb{R}\right)$ to the system $(21)-$ (23) satisfying

$$
|\mathbf{z}(t, \cdot)|_{H^{2}\left(\left(0, x_{0}\right) ; \mathbb{R}^{2}\right)}+\left|x_{s}(t)-x_{0}\right|<\beta
$$

and such that (111) holds on $\left(0, T_{1}\right)$ in a distribution sense. From (113), Lemma 4.1 and (112),

$$
\left|\mathbf{z}\left(T_{1}, \cdot\right)\right|_{H^{2}\left(\left(0, x_{0}\right) ; \mathbb{R}^{2}\right)} \leqslant \delta_{0}\left(T_{1}\right), \quad\left|x_{s}\left(T_{1}\right)-x_{0}\right| \leqslant \delta_{0}\left(T_{1}\right)
$$

Moreover, the compatibility conditions hold now at time $t=T_{1}$ instead of $t=0$. Thus, from Lemma 3.1 there exists a unique $\left(\mathbf{z}, x_{s}\right) \in C^{0}\left(\left[T_{1}, 2 T_{1}\right] ; H^{2}\left(\left(0, x_{0}\right) ; \mathbb{R}^{2}\right)\right) \times C^{1}\left(\left[T_{1}, 2 T_{1}\right] ; \mathbb{R}\right)$ solution of (21)(23) on $\left[T_{1}, 2 T_{1}\right]$ and (111) holds on $\left(T_{1}, 2 T_{1}\right)$ in a distribution sense. One can repeat this analysis on $\left[j T_{1},(j+1) T_{1}\right]$ where $j \in \mathbb{N}^{*} \backslash\{1\}$. Setting $\delta^{*}=\delta_{0}\left(T_{1}\right)$, we get that (111) holds on $(0, T)$ for any $T>0$ in a distribution sense along the $C^{0}\left([0, T] ; H^{2}\left(\left(0, x_{0}\right) ; \mathbb{R}^{2}\right)\right) \times C^{1}([0, T] ; \mathbb{R})$ solutions of the system (21)-(23). In fact, it also implies the global existence and uniqueness of $\left(\mathbf{z}, x_{s}\right) \in$ $C^{0}\left([0,+\infty) ; H^{2}\left(\left(0, x_{0}\right) ; \mathbb{R}^{2}\right)\right) \times C^{1}([0,+\infty) ; \mathbb{R})$ solution of $(21)-(23)$ and the fact that (111) holds on $(0,+\infty)$. This concludes the proof of Theorem 4.1 . 


\section{Extension to a general convex flux}

We can in fact extend this method to a more general convex flux. Let $f \in C^{3}(\mathbb{R})$ be a convex function, and consider the equation

$$
\partial_{t} y+\partial_{x}(f(y))=0 .
$$

For this conservation law, the Rankine-Hugoniot condition becomes

$$
\dot{x}_{s}=\frac{f\left(y\left(t, x_{s}(t)^{+}\right)\right)-f\left(y\left(t, x_{s}(t)^{-}\right)\right)}{y\left(t, x_{s}(t)^{+}\right)-y\left(t, x_{s}(t)^{-}\right)},
$$

and, let $\left(y^{*}, x_{0}\right)$ be an entropic shock steady state of $(115)-(116)$, without loss of generality we can assume that $y^{*}\left(x_{0}^{+}\right)=-1$ and $y^{*}\left(x_{0}^{-}\right)=1$, thus $f(1)=f(-1)$. Then, for any $x_{0} \in(0, L)$, we have the following result:

Theorem 5.1. Let $f \in C^{3}(\mathbb{R})$ be a convex function such that $f(1)=f(-1)$ and assume in addition that

$$
f^{\prime}(1) \geqslant 1 \text { and }\left|f^{\prime}(-1)\right| \geqslant 1 .
$$

Let $\gamma>0$. If the following conditions hold

$$
\begin{aligned}
& b_{1} \in\left(\frac{2 \gamma e^{-\gamma x_{0}}}{f^{\prime}(1)+\left|f^{\prime}(-1)\right|}, \frac{\gamma e^{-\gamma x_{0}}}{1-e^{-\gamma x_{0}}}\right), b_{2} \in\left(\frac{2 \gamma e^{-\gamma\left(L-x_{0}\right)}}{f^{\prime}(1)+\left|f^{\prime}(-1)\right|}, \frac{\gamma e^{-\gamma\left(L-x_{0}\right)}}{1-e^{-\gamma\left(L-x_{0}\right)}}\right), \\
& k_{1}^{2}<e^{-\gamma x_{0}}\left(1-f^{\prime}(1) \frac{b_{1}}{\gamma}\left(b_{1} \frac{1-e^{-\gamma x_{0}}}{\gamma e^{-\gamma x_{0}}}+b_{2} \frac{1-e^{-\gamma\left(L-x_{0}\right)}}{\gamma e^{-\gamma\left(L-x_{0}\right)}}\right)\right), \\
& k_{2}^{2}<e^{-\gamma\left(L-x_{0}\right)}\left(1-\left|f^{\prime}(-1)\right| \frac{b_{2}}{\gamma}\left(b_{1} \frac{1-e^{-\gamma x_{0}}}{\gamma e^{-\gamma x_{0}}}+b_{2} \frac{1-e^{-\gamma\left(L-x_{0}\right)}}{\gamma e^{-\gamma\left(L-x_{0}\right)}}\right)\right),
\end{aligned}
$$

then the steady state $\left(y^{*}, x_{0}\right)$ of the system (115), (116), (3), (8) is exponentially stable for the $H^{2}$ norm with decay rate $\gamma / 4$.

One can use exactly the same method as previously. We give in Appendix B a way to adapt the proof of Theorem 4.1 .

Remark 9. One could wonder why we require condition (117). This condition ensures that there always exist parameters $b_{i}$ and $k_{i}$ satisfying (118). 


\section{Conclusion and Open problems}

The stabilization of shock-free regular solutions of quasilinear hyperbolic systems has been the subject of a large number of publications in the recent scientific literature. In contrast, there are no results concerning the Lyapunov stability of solutions with jump discontinuities, although they occur naturally in the form of shock waves or hydraulic jumps in many applications of fluid dynamics. For instance, the inviscid Burgers equation provides a simple scalar example of a hyperbolic system having natural solutions with jump discontinuities. The main contribution of this paper is precisely to address the issue of the boundary exponential feedback stabilization of an unstable shock steady state for the Burgers equation over a bounded interval. Our strategy to solve the problem relies on introducing a change of variables which allows to transform the scalar Burgers equation with shock wave solutions into an equivalent $2 \times 2$ quasilinear hyperbolic system having shock-free solutions over a bounded interval. Then, by a Lyapunov approach, we show that, for appropriately chosen boundary conditions, the exponential stability in $H^{2}$-norm of the steady state can be achieved with an arbitrary decay rate and with an exact exponential stabilization of the desired shock location. Compared with previous results in the literature for classical solutions of quasilinear hyperbolic systems, the selection of an appropriate Lyapunov function is challenging because the equivalent system is parameterized by the time-varying position of the jump discontinuity. In particular, the standard quadratic Lyapunov function used in the book [3] has to be augmented with suitable extra terms for the analysis of the stabilization of the jump position. Based on the result, some open questions could be addressed. Could these results be generalized to any convex flux, especially when (117) is not satisfied? As we show the rapid stabilization result, is it possible to obtain finite time stabilization? Could we replace the left/right state at the shock by measurements nearby or by averages close to the shock? If not, could the error on both the state and shock location be bounded?

\section{Acknowledgement}

The authors would like to thank Tatsien Li and Sébastien Boyaval for their constant support. They would like also to thank National Natural Science Foundation of China (No. 11771336), ETH-ITS, ETH-FIM, ANR project Finite4SoS (No.ANR 15-CE23-0007), LIASFMA and the French Corps des IPEF for their financial support.

\section{Appendix}

\section{A Proof of Lemma 3.1}

Proof. We adapt the fixed point method used in [3, Appendix B] (see also [25, 31]). We first deal with the case where

$$
T \in\left(0, \min \left(x_{0}, L-x_{0}\right)\right) .
$$

For any $\nu>0, x_{s 0} \in \mathbb{R}$ and $\mathbf{z}^{0} \in H^{2}\left(\left(0, x_{0}\right) ; \mathbb{R}^{2}\right)$, let $C_{\nu}\left(\mathbf{z}^{0}, x_{s 0}\right)$ be the set of

$$
\mathbf{z} \in L^{\infty}\left((0, T) ; H^{2}\left(\left(0, x_{0}\right) ; \mathbb{R}^{2}\right)\right) \cap W^{1, \infty}\left((0, T) ; H^{1}\left(\left(0, x_{0}\right) ; \mathbb{R}^{2}\right)\right) \cap W^{2, \infty}\left((0, T) ; L^{2}\left(\left(0, x_{0}\right) ; \mathbb{R}^{2}\right)\right)
$$

such that

$$
\begin{aligned}
& |\mathbf{z}|_{L^{\infty}\left((0, T) ; H^{2}\left(\left(0, x_{0}\right) ; \mathbb{R}^{2}\right)\right)} \leqslant \nu, \\
& |\mathbf{z}|_{W^{1, \infty}\left((0, T) ; H^{1}\left(\left(0, x_{0}\right) ; \mathbb{R}^{2}\right)\right)} \leqslant \nu, \\
& |\mathbf{z}|_{W^{2, \infty}\left((0, T) ; L^{2}\left(\left(0, x_{0}\right) ; \mathbb{R}^{2}\right)\right)} \leqslant \nu, \\
& \left.\mathbf{z}\left(\cdot, x_{0}\right) \in H^{2}\left((0, T) ; \mathbb{R}^{2}\right)\right), \quad\left|\mathbf{z}\left(\cdot, x_{0}\right)\right|_{\left.H^{2}\left((0, T) ; \mathbb{R}^{2}\right)\right)} \leqslant \nu^{2}, \\
& \mathbf{z}(0, \cdot)=\mathbf{z}^{0}, \\
& \mathbf{z}_{t}(0, \cdot)=-A\left(\mathbf{z}^{0}, \cdot, x_{s}\left(\mathbf{z}\left(\cdot, x_{0}\right)\right)(0)\right) \mathbf{z}_{x}^{0},
\end{aligned}
$$

where we write $x_{s}\left(\mathbf{z}\left(\cdot, x_{0}\right)\right)(t)$ in order to emphasize its dependence on $\mathbf{z}\left(\cdot, x_{0}\right)$ in the following proof and

$$
x_{s}\left(\mathbf{z}\left(\cdot, x_{0}\right)\right)(t)=: x_{s 0}+\int_{0}^{t} \frac{z_{1}\left(s, x_{0}\right)+z_{2}\left(s, x_{0}\right)}{2} d s .
$$


In 125 ,

$$
A\left(\mathbf{z}, x, x_{s}\left(\mathbf{z}\left(\cdot, x_{0}\right)\right)(t)\right)=\left(\begin{array}{cc}
a_{1}\left(\mathbf{z}, x, x_{s}\left(\mathbf{z}\left(\cdot, x_{0}\right)\right)(t)\right) & 0 \\
0 & a_{2}\left(\mathbf{z}, x, x_{s}\left(\mathbf{z}\left(\cdot, x_{0}\right)\right)(t)\right)
\end{array}\right)
$$

with

$$
\begin{aligned}
& a_{1}\left(\mathbf{z}, x, x_{s}\left(\mathbf{z}\left(\cdot, x_{0}\right)\right)(t)\right)=\left(1+z_{1}(t, x)-x \frac{z_{1}\left(t, x_{0}\right)+z_{2}\left(t, x_{0}\right)}{2 x_{0}}\right) \frac{x_{0}}{x_{s}\left(\mathbf{z}\left(\cdot, x_{0}\right)\right)(t)}, \\
& a_{2}\left(\mathbf{z}, x, x_{s}\left(\mathbf{z}\left(\cdot, x_{0}\right)\right)(t)\right)=\left(1-z_{2}(t, x)+x \frac{z_{1}\left(t, x_{0}\right)+z_{2}\left(t, x_{0}\right)}{2 x_{0}}\right) \frac{x_{0}}{L-x_{s}\left(\mathbf{z}\left(\cdot, x_{0}\right)\right)(t)} .
\end{aligned}
$$

The set $C_{\nu}\left(\mathbf{z}^{0}, x_{s 0}\right)$ is not empty and is a closed subset of $L^{\infty}\left((0, T) ; L^{2}\left((0, L) ; \mathbb{R}^{2}\right)\right)$ provided that $\left|\mathbf{z}^{0}\right|_{H^{2}\left(\left(0, x_{0}\right) ; \mathbb{R}^{2}\right)} \leqslant \delta$ and $\left|x_{s 0}-x_{0}\right| \leqslant \delta$, with $\delta$ sufficiently small (see for instance [3, Appendix B]).

Let us define a mapping:

$$
\begin{array}{cr}
\mathcal{F}: C_{\nu}\left(\mathbf{z}^{0}, x_{s 0}\right) \longrightarrow L^{\infty}\left((0, T) ; H^{2}\left(\left(0, x_{0}\right) ; \mathbb{R}^{2}\right)\right) \cap W^{1, \infty}\left((0, T) ; H^{1}\left(\left(0, x_{0}\right) ; \mathbb{R}^{2}\right)\right) \\
\mathbf{v}=\left(v_{1}, v_{2}\right)^{T} \longmapsto \mathcal{F}(\mathbf{v})=\mathbf{z}=\left(z_{1}, z_{2}\right)^{T} & \cap W^{2, \infty}\left((0, T) ; L^{2}\left(\left(0, x_{0}\right) ; \mathbb{R}^{2}\right)\right)
\end{array}
$$

where $\mathbf{z}$ is the solution of the linear hyperbolic equation

$$
\begin{aligned}
& \mathbf{z}_{t}+A\left(\mathbf{v}, x, x_{s}\left(\mathbf{v}\left(\cdot, x_{0}\right)\right)(t)\right) \mathbf{z}_{x}=0, \\
& \mathbf{z}(0, x)=\mathbf{z}^{0}(x)
\end{aligned}
$$

with boundary conditions

$$
\begin{aligned}
& z_{1}(t, 0)=k_{1} z_{1}\left(t, x_{0}\right)+b_{1} \psi(t), \\
& z_{2}(t, 0)=k_{2} z_{2}\left(t, x_{0}\right)+b_{2} \psi(t),
\end{aligned}
$$

where

$$
\psi(t)=x_{0}-x_{s}\left(\mathbf{v}\left(\cdot, x_{0}\right)\right)(t) .
$$

In the following, we will treat $z_{1}$ in details. For the sake of simplicity, we denote

$$
f_{1}(t, x):=a_{1}\left(\mathbf{v}(t, x), x, x_{s}\left(\mathbf{v}\left(\cdot, x_{0}\right)\right)(t)\right) .
$$

It is easy to check from (128) that if $\nu$ is sufficiently small, then $f_{1}(t, x)$ is strictly positive for any $(t, x) \in[0, T] \times\left[0, x_{0}\right]$. Let us now define the characteristic curve $\xi_{1}(s ; t, x)$ passing through $(t, x)$ as

$$
\begin{aligned}
& \frac{d \xi_{1}(s ; t, x)}{d s}=f_{1}\left(s, \xi_{1}(s ; t, x)\right), \\
& \xi_{1}(t ; t, x)=x .
\end{aligned}
$$

One can see that for every $(t, x) \in[0, T] \times\left[0, x_{0}\right], \xi_{1}(\cdot ; t, x)$ is uniquely defined on some closed interval in $[0, T]$. From (119), only two cases can occur (see Figure 2$)$ : If $\xi_{1}(t ; 0,0)<x \leqslant x_{0}$, there exists $\beta_{1} \in\left[0, x_{0}\right]$ depending on $(t, x)$ such that

$$
\beta_{1}=\xi_{1}(0 ; t, x)
$$

If $0<x<\xi_{1}(t ; 0,0)$, there exists $\alpha_{1} \in[0, t]$ depending on $(t, x)$ such that

$$
\xi_{1}\left(\alpha_{1} ; t, x\right)=0,
$$

and in this case, there exists $\gamma_{1} \in\left[0, x_{0}\right]$ depending on $\alpha_{1}$ such that

$$
\gamma_{1}=\xi_{1}\left(0 ; \alpha_{1}, x_{0}\right)
$$

Moreover, we have the following lemma which will be used in the estimations hereafter (the proof can be found at the end of this appendix). 
Lemma A.1. There exist $\nu_{0}>0$ and $C>0$ such that, for any $T$ satisfying $(119)$, for any $\nu \in\left(0, \nu_{0}\right]$ and for a.e. $t \in(0, T)$, we have

$$
\begin{aligned}
& \left|f_{1}(t, \cdot)\right|_{0} \leqslant C, \quad\left|f_{1 x}(t, \cdot)\right|_{0} \leqslant C \nu, \quad\left|f_{1 t}(t, \cdot)\right|_{0} \leqslant C \nu, \\
& \left|\partial_{x} \xi_{1}(s ; t, \cdot)\right|_{0} \leqslant C, \quad\left|\partial_{t} \xi_{1}(s ; t, \cdot)\right|_{0} \leqslant C, \quad s \in[0, t] \\
& \left|\partial_{x} \beta_{1}(t, \cdot)\right|_{0} \leqslant C, \quad\left|\left(\partial_{x} \beta_{1}(t, \cdot)\right)^{-1}\right|_{0} \leqslant C, \\
& \left|\partial_{t} \beta_{1}(t, \cdot)\right|_{0} \leqslant C, \quad\left|\left(\partial_{t} \beta_{1}(t, \cdot)\right)^{-1}\right|_{0} \leqslant C \\
& \left|\partial_{x} \alpha_{1}(t, \cdot)\right|_{0} \leqslant C, \quad\left|\left(\partial_{x} \alpha_{1}(t, \cdot)\right)^{-1}\right|_{0} \leqslant C, \\
& \left|\partial_{x} \gamma_{1}(t, \cdot)\right|_{0} \leqslant C, \quad\left|\left(\partial_{x} \gamma_{1}(t, \cdot)\right)^{-1}\right|_{0} \leqslant C \\
& \int_{0}^{T}\left|\partial_{t t} \beta_{1}\left(t, x_{0}\right)\right|^{2} d t \leqslant C \nu \\
& \int_{0}^{x_{0}}\left|\partial_{x x} \alpha_{1}(t, x)\right|^{2} d x \leqslant C \nu \\
& \int_{0}^{x_{0}}\left|\partial_{x x} \beta_{1}(t, x)\right|^{2} d x \leqslant C \nu \\
& \int_{0}^{x_{0}}\left|\partial_{x x} \gamma_{1}(t, x)\right|^{2} d x \leqslant C \nu .
\end{aligned}
$$

In these inequalities, and hereafter in this section, $|f|_{0}$ denotes the $C^{0}$-norm of a function $f$ with respect to its variable and $C$ may depend on $x_{0}, x_{s 0}, \nu_{0}, k_{1}, k_{2}, b_{1}$ and $b_{2}$, but is independent of $\nu$, $T, \mathbf{v}$ and $\mathbf{z}$.

Our goal is now to use a fixed point argument to show the existence and uniqueness of the solution to (21)-(23). Firstly, we show that for $\nu$ and $\delta$ sufficiently small, $\mathcal{F}$ maps $C_{\nu}\left(\mathbf{z}^{0}, x_{s 0}\right)$ into itself, i.e.,

$$
\mathcal{F}\left(C_{\nu}\left(\mathbf{z}^{0}, x_{s 0}\right)\right) \subset C_{\nu}\left(\mathbf{z}^{0}, x_{s 0}\right) .
$$

Then, in a second step, we prove that $\mathcal{F}$ is a contraction mapping.

1) $\mathcal{F}$ maps $C_{\nu}\left(\mathbf{z}^{0}, x_{s 0}\right)$ into itself.

For any $\mathbf{v} \in C_{\nu}\left(\mathbf{z}^{0}, x_{s 0}\right)$, let $\mathbf{z}=\mathcal{F}(\mathbf{v})$, we prove that $\mathbf{z} \in C_{\nu}\left(\mathbf{z}^{0}, x_{s 0}\right)$. By the definition of $\mathcal{F}$ in 130], using the method of characteristics, we can solve (131) to (134) for $z_{1}$ and obtain that

$$
z_{1}(t, x)=\left\{\begin{array}{l}
k_{1} z_{1}^{0}\left(\gamma_{1}\right)+b_{1} \psi\left(\alpha_{1}\right), \quad 0<x<\xi_{1}(t ; 0,0), \\
z_{1}^{0}\left(\beta_{1}\right), \quad \xi_{1}(t ; 0,0)<x<x_{0} .
\end{array}\right.
$$

Obviously $\mathbf{z}$ verifies the properties $124-(125)$. Next, we prove that $\mathbf{z}$ verifies the property 123 . Using the change of variables and from (144), we have

$$
\int_{0}^{T} z_{1}\left(t, x_{0}\right)^{2} d t=\int_{0}^{T} z_{1}^{0}\left(\beta_{1}\left(t, x_{0}\right)\right)^{2} d t \leqslant C \int_{0}^{x_{0}}\left(z_{1}^{0}(x)\right)^{2} d x .
$$

In (152) and hereafter, $C$ denotes various constants that may depend on $x_{0}, x_{s 0}, \nu_{0}, k_{1}, k_{2}, b_{1}$ and $b_{2}$, but are independent of $\nu, T, \mathbf{v}$ and $\mathbf{z}$. Similarly, by [144, we obtain

$$
\int_{0}^{T} z_{1 t}\left(t, x_{0}\right)^{2} d t=\int_{0}^{T}\left(z_{1 x}^{0}\left(\beta_{1}\left(t, x_{0}\right)\right) \partial_{t} \beta_{1}\left(t, x_{0}\right)\right)^{2} d t \leqslant C \int_{0}^{T}\left(z_{1 x}^{0}(x)\right)^{2} d x .
$$

From (147) and using Sobolev inequality, one has

$$
\begin{aligned}
\int_{0}^{T} z_{1 t t}\left(t, x_{0}\right)^{2} d t & =\int_{0}^{T}\left(z_{1 x x}^{0}\left(\beta_{1}\left(t, x_{0}\right)\right)\left(\partial_{t} \beta_{1}\left(t, x_{0}\right)\right)^{2}+z_{1 x}^{0}\left(\beta_{1}\left(t, x_{0}\right)\right) \partial_{t t} \beta_{1}\left(t, x_{0}\right)\right)^{2} d t \\
& \leqslant C \int_{0}^{x_{0}}\left(z_{1 x x}^{0}(x)\right)^{2} d x+2\left|z_{1 x}^{0}\right|_{0}^{2} \int_{0}^{T}\left(\partial_{t t} \beta_{1}\left(t, x_{0}\right)\right)^{2} d t \\
& \leqslant C\left|z_{1}^{0}\right|_{H^{2}\left(\left(0, x_{0}\right) ; \mathbb{R}\right) .}^{2}
\end{aligned}
$$


Combining 152 -154, we get

$$
\left|z_{1}\left(\cdot, x_{0}\right)\right|_{H^{2}((0, T) ; \mathbb{R})} \leqslant C\left|z_{1}^{0}\right|_{H^{2}\left(\left(0, x_{0}\right) ; \mathbb{R}\right)} .
$$

Applying similar estimate to $z_{2}$ gives

$$
\left|z_{2}\left(\cdot, x_{0}\right)\right|_{H^{2}((0, T) ; \mathbb{R})} \leqslant C\left|z_{2}^{0}\right|_{H^{2}\left(\left(0, x_{0}\right) ; \mathbb{R}\right)} .
$$

From (155) and (156), we can select $\delta$ sufficiently small such that

$$
\left|\mathbf{z}\left(\cdot, x_{0}\right)\right|_{H^{2}\left((0, T) ; \mathbb{R}^{2}\right)} \leqslant \nu^{2},
$$

which shows both the regularity and the boundedness property $(123)$. We can again use the method of characteristics to prove properties $120-122$. For a.e. $t \in(0, T)$,

$$
\begin{gathered}
z_{1 x}(t, x)=\left\{\begin{array}{l}
k_{1} z_{1 x}^{0}\left(\gamma_{1}\right) \partial_{x} \gamma_{1}+b_{1} \dot{\psi}\left(\alpha_{1}\right) \partial_{x} \alpha_{1}, \quad 0<x<\xi_{1}(t ; 0,0), \\
z_{1 x}^{0}\left(\beta_{1}\right) \partial_{x} \beta_{1}, \quad \xi_{1}(t ; 0,0)<x<x_{0} .
\end{array}\right. \\
z_{1 x x}(t, x)=\left\{\begin{array}{l}
k_{1} z_{1 x}^{0}\left(\gamma_{1}\right) \partial_{x x} \gamma_{1}+k_{1} z_{1 x x}^{0}\left(\gamma_{1}\right)\left(\partial_{x} \gamma_{1}\right)^{2} \\
+b_{1} \ddot{\psi}\left(\alpha_{1}\right)\left(\partial_{x} \alpha_{1}\right)^{2}+b_{1} \dot{\psi}\left(\alpha_{1}\right) \partial_{x x} \alpha_{1}, \quad 0<x<\xi_{1}(t ; 0,0), \\
z_{1 x}^{0}\left(\beta_{1}\right) \partial_{x x} \beta_{1}+z_{1 x x}^{0}\left(\beta_{1}\right)\left(\partial_{x} \beta_{1}\right)^{2}, \quad \xi_{1}(t ; 0,0)<x<x_{0} .
\end{array}\right.
\end{gathered}
$$

Note that the last equation is true in distribution sense but shows that $z_{1} \in L^{\infty}\left((0, T) ; H^{2}\left(\left(0, x_{0}\right) ; \mathbb{R}\right)\right)$. We first estimate $|\mathbf{z}|_{L^{\infty}\left((0, T) ; H^{2}\left(\left(0, x_{0}\right) ; \mathbb{R}^{2}\right)\right)}$. From 126) and 135), using Sobolev inequality, we get

$$
\begin{aligned}
& |\psi|_{0} \leqslant\left|x_{s 0}-x_{0}\right|+C\left|\mathbf{v}\left(\cdot, x_{0}\right)\right|_{H^{2}\left((0, T) ; \mathbb{R}^{2}\right)}, \\
& |\dot{\psi}|_{0} \leqslant C\left|\mathbf{v}\left(\cdot, x_{0}\right)\right|_{H^{2}\left((0, T) ; \mathbb{R}^{2}\right)} \\
& |\ddot{\psi}|_{0} \leqslant C\left|\mathbf{v}\left(\cdot, x_{0}\right)\right|_{H^{2}\left((0, T) ; \mathbb{R}^{2}\right)} .
\end{aligned}
$$

From (151), 158) and (159), we can compute directly using (143), 145)-146) and (148)-(150) that

$$
\begin{aligned}
\int_{0}^{x_{0}} z_{1}^{2} d x \leqslant & \left(\left|\left(\partial_{x} \beta_{1}(t, \cdot)\right)^{-1}\right|_{0}+2 k_{1}^{2}\left|\left(\partial_{x} \gamma_{1}(t, \cdot)\right)^{-1}\right|_{0}\right) \int_{0}^{x_{0}}\left(z_{1}^{0}(x)\right)^{2} d x+2 b_{1}^{2} x_{0}|\psi|_{0}^{2} \\
\leqslant & C\left(\left|z_{1}^{0}\right|_{H^{2}\left(\left(0, x_{0}\right) ; \mathbb{R}\right)}^{2}+\left|x_{s 0}-x_{0}\right|^{2}+\left|\mathbf{v}\left(\cdot, x_{0}\right)\right|_{H^{2}\left((0, T) ; \mathbb{R}^{2}\right)}^{2}\right) \\
\int_{0}^{x_{0}} z_{1 x}^{2} d x \leqslant & \left(\left|\partial_{x} \beta_{1}(t, \cdot)\right|_{0}+2 k_{1}^{2}\left|\partial_{x} \gamma_{1}(t, \cdot)\right|_{0}\right) \int_{0}^{x_{0}}\left(z_{1 x}^{0}(x)\right)^{2} d x+2 x_{0} b_{1}^{2}|\dot{\psi}|_{0}^{2}\left|\partial_{x} \alpha_{1}(t, \cdot)\right|_{0}^{2} \\
\leqslant & C\left(\left|z_{1}^{0}\right|_{H^{2}\left(\left(0, x_{0}\right) ; \mathbb{R}\right)}^{2}+\left|\mathbf{v}\left(\cdot, x_{0}\right)\right|_{H^{2}\left((0, T) ; \mathbb{R}^{2}\right)}^{2}\right) \\
\int_{0}^{x_{0}} z_{1 x x}^{2} d x \leqslant & \left(2\left|\partial_{x} \beta_{1}(t, \cdot)\right|_{0}^{3}+4 k_{1}^{2}\left|\partial_{x} \gamma_{1}(t, \cdot)\right|_{0}^{3}\right) \int_{0}^{x_{0}}\left(z_{1 x x}^{0}\right)^{2} d x \\
& +2\left|z_{1 x}^{0}\right|_{0}^{2} \int_{0}^{x_{0}}\left|\partial_{x x} \beta_{1}(t, x)\right|^{2} d x+4 k_{1}^{2}\left|z_{1 x}^{0}\right|_{0}^{2} \int_{0}^{x_{0}}\left|\partial_{x x} \gamma_{1}(t, x)\right|^{2} d x \\
& +4 b_{1}^{2}\left|\partial_{x} \alpha_{1}(t, x)\right|_{0}^{4} \int_{0}^{x_{0}}\left|\ddot{\psi}\left(\alpha_{1}(t, x)\right)\right|^{2} d x+4 b_{1}^{2}|\dot{\psi}|_{0}^{2} \int_{0}^{x_{0}}\left|\partial_{x x} \alpha_{1}(t, x)\right|^{2} d x \\
\leqslant & C\left(\left|z_{1}^{0}\right|_{H^{2}\left(\left(0, x_{0}\right) ; \mathbb{R}\right)}^{2}+\left|\mathbf{v}\left(\cdot, x_{0}\right)\right|_{H^{2}\left((0, T) ; \mathbb{R}^{2}\right)}^{2}\right) .
\end{aligned}
$$

Combining (163)-165), we obtain

$$
\left|z_{1}(t, \cdot)\right|_{H^{2}\left(\left(0, x_{0}\right) ; \mathbb{R}\right)} \leqslant C\left(\left|z_{1}^{0}\right|_{H^{2}\left(\left(0, x_{0}\right) ; \mathbb{R}\right)}+\left|x_{s 0}-x_{0}\right|+\left|\mathbf{v}\left(\cdot, x_{0}\right)\right|_{H^{2}\left((0, T) ; \mathbb{R}^{2}\right)}\right),
$$

Similarly, one can get

$$
\left|z_{2}(t, \cdot)\right|_{H^{2}\left(\left(0, x_{0}\right) ; \mathbb{R}\right)} \leqslant C\left(\left|z_{2}^{0}\right|_{H^{2}\left(\left(0, x_{0}\right) ; \mathbb{R}\right)}+\left|x_{s 0}-x_{0}\right|+\left|\mathbf{v}\left(\cdot, x_{0}\right)\right|_{H^{2}\left((0, T) ; \mathbb{R}^{2}\right)}\right) .
$$

Noticing from $\mathbf{v} \in C_{\nu}\left(\mathbf{z}^{0}, x_{s 0}\right)$ that

$$
\left|\mathbf{v}\left(\cdot, x_{0}\right)\right|_{H^{2}\left((0, T) ; \mathbb{R}^{2}\right)} \leqslant \nu^{2},
$$


thus by selecting $\delta$ and $\nu \in\left(0, \nu_{0}\right]$ sufficiently small, in addition to the previous hypothesis on $\delta$, we have indeed

$$
|\mathbf{z}(t, \cdot)|_{H^{2}\left(\left(0, x_{0}\right) ; \mathbb{R}^{2}\right)} \leqslant \nu, \quad \text { a.e. } \quad t \in(0, T),
$$

which proves 120 . The same method as to prove (168) enables us to show that $z_{1}$ verifies also (121) and 122 . One only has to realize that

$$
\begin{gathered}
z_{1 t}(t, x)=\left\{\begin{array}{l}
k_{1} z_{1 x}^{0}\left(\gamma_{1}\right) \partial_{t} \gamma_{1}+b_{1} \dot{\psi}\left(\alpha_{1}\right) \partial_{t} \alpha_{1}, \quad 0<x<\xi_{1}(t ; 0,0) \\
z_{1 x}^{0}\left(\beta_{1}\right) \partial_{t} \beta_{1}, \quad \xi_{1}(t ; 0,0)<x<x_{0}
\end{array}\right. \\
z_{1 t t}(t, x)=\left\{\begin{array}{l}
k_{1} z_{1 x}^{0}\left(\gamma_{1}\right) \partial_{t t} \gamma+k_{1} z_{1 x x}^{0}\left(\gamma_{1}\right)\left(\partial_{t} \gamma_{1}\right)^{2}+b_{1} \ddot{\psi}\left(\alpha_{1}\right)\left(\partial_{t} \alpha_{1}\right)^{2}+b_{1} \dot{\psi}\left(\alpha_{1}\right) \partial_{t t} \alpha_{1}, \quad 0<x<\xi_{1}(t ; 0,0), \\
z_{1 x}^{0}\left(\beta_{1}\right) \partial_{t t} \beta_{1}+z_{1 x x}^{0}\left(\beta_{1}\right)\left(\partial_{t} \beta_{1}\right)^{2}, \quad \xi_{1}(t ; 0,0)<x<x_{0}
\end{array}\right. \\
z_{1 t x}(t, x)=\left\{\begin{array}{c}
k_{1} z_{1 x}^{0}\left(\gamma_{1}\right) \partial_{x}\left(\partial_{t} \gamma_{1}\right)+k_{1} z_{1 x x}^{0}\left(\gamma_{1}\right)\left(\partial_{x} \gamma_{1} \partial_{t} \gamma_{1}\right) \quad 0<x<\xi_{1}(t ; 0,0) \\
\left.+b_{1} \ddot{\psi}\left(\alpha_{1}\right)\left(\partial_{t} \alpha_{1} \partial_{x} \alpha_{1}\right)+b_{1} \dot{\psi}\left(\alpha_{1}\right) \partial_{x}\left(\partial_{t} \alpha_{1}\right), \quad 0<x_{1}\right) \\
z_{1 x}^{0}\left(\beta_{1}\right) \partial_{x}\left(\partial_{t} \beta_{1}\right)+z_{1 x x}^{0}\left(\beta_{1}\right)\left(\partial_{x} \beta_{1} \partial_{t} \beta_{1}\right), \quad \xi_{1}(t ; 0,0)<x<x_{0}
\end{array}\right.
\end{gathered}
$$

and to estimate $\int_{0}^{\xi_{1}(t ; 0,0)}\left|\partial_{t t} \alpha_{1}\right|^{2} d x, \int_{\xi_{1}(t ; 0,0)}^{x_{0}}\left|\partial_{t t} \beta_{1}\right|^{2} d x, \int_{0}^{\xi_{1}(t ; 0,0)}\left|\partial_{t t} \gamma_{1}\right|^{2} d x, \int_{0}^{\xi_{1}(t ; 0,0)}\left|\partial_{x}\left(\partial_{t} \alpha_{1}\right)\right|^{2} d x$, $\int_{\xi_{1}(t, 0,0)}^{x_{0}}\left|\partial_{x}\left(\partial_{t} \beta_{1}\right)\right|^{2} d x$ and $\int_{0}^{\xi_{1}(t ; 0,0)}\left|\partial_{x}\left(\partial_{t} \gamma_{1}\right)\right|^{2} d x$ similarly as in 148-150 using the fact that $\mathbf{v}$ belongs to $L^{\infty}\left((0, T) ; H^{2}\left(\left(0, x_{0}\right) ; \mathbb{R}^{2}\right)\right) \cap W^{1, \infty}\left((0, T) ; H^{1}\left(\left(0, x_{0}\right) ; \mathbb{R}^{2}\right)\right) \cap W^{2, \infty}\left((0, T) ; L^{2}\left(\left(0, x_{0}\right) ; \mathbb{R}^{2}\right)\right)$ with bound $\nu$ in these norms.

We can clearly perform similar estimates for $z_{2}$. Consequently there exist $\delta$ and $\nu_{1} \in\left(0, \nu_{0}\right]$ sufficiently small depending only on $C$ such that, for any $\nu \in\left(0, \nu_{1}\right], \mathbf{z}=\mathcal{F}(\mathbf{v})$ verifies properties 120 -125 and therefore $\mathcal{F}\left(C_{\nu}\left(\mathbf{z}^{0}, x_{s 0}\right)\right) \subset C_{\nu}\left(\mathbf{z}^{0}, x_{s 0}\right)$.

2) $\mathcal{F}$ is a contraction mapping.

Next, we prove that $\mathcal{F}$ is a contraction mapping satisfying the following inequality:

$$
\begin{aligned}
& |\mathcal{F}(\mathbf{v})-\mathcal{F}(\overline{\mathbf{v}})|_{L^{\infty}\left((0, T) ; L^{2}\left(\left(0, x_{0}\right) ; \mathbb{R}^{2}\right)\right)}+M\left|\mathcal{F}(\mathbf{v})\left(\cdot, x_{0}\right)-\mathcal{F}(\overline{\mathbf{v}})\left(\cdot, x_{0}\right)\right|_{L^{2}\left((0, T) ; \mathbb{R}^{2}\right)} \\
\leqslant & \frac{1}{2}|\mathbf{v}-\overline{\mathbf{v}}|_{L^{\infty}\left((0, T) ; L^{2}\left(\left(0, x_{0}\right) ; \mathbb{R}^{2}\right)\right)}+\frac{M}{2}\left|\mathbf{v}\left(\cdot, x_{0}\right)-\overline{\mathbf{v}}\left(\cdot, x_{0}\right)\right|_{L^{2}\left((0, T) ; \mathbb{R}^{2}\right),}
\end{aligned}
$$

where $M>0$ is a constant. We start with $z_{1}$, and with the estimate of $\left|z_{1}-\bar{z}_{1}\right|_{L^{\infty}\left((0, T) ; L^{2}\left(\left(0, x_{0}\right) ; \mathbb{R}\right)\right)}$. For any chosen $\mathbf{v}$ and $\overline{\mathbf{v}}$ from $C_{\nu}\left(\mathbf{z}^{0}, x_{s 0}\right)$, without loss of generality, we may assume that $\xi_{1}(t ; 0,0)<$ $\bar{\xi}_{1}(t ; 0,0)$, where $\bar{\xi}_{1}$ is the characteristic defined in (137) associated to $\overline{\mathbf{v}}$. From (151), we have

$$
\begin{aligned}
& \int_{0}^{x_{0}}\left|z_{1}(t, x)-\bar{z}_{1}(t, x)\right|^{2} d x \\
= & \int_{0}^{\xi_{1}(t ; 0,0)}\left|k_{1} z_{1}^{0}\left(\gamma_{1}\right)-k_{1} z_{1}^{0}\left(\bar{\gamma}_{1}\right)+b_{1} \psi\left(\alpha_{1}\right)-b_{1} \bar{\psi}\left(\bar{\alpha}_{1}\right)\right|^{2} d x \\
& +\int_{\xi_{1}(t ; 0,0)}^{\bar{\xi}_{1}(t ; 0,0)}\left|z_{1}^{0}\left(\beta_{1}\right)-\left(k_{1} z_{1}^{0}\left(\bar{\gamma}_{1}\right)+b_{1} \bar{\psi}\left(\bar{\alpha}_{1}\right)\right)\right|^{2} d x+\int_{\bar{\xi}_{1}(t ; 0,0)}^{x_{0}}\left|z_{1}^{0}\left(\beta_{1}\right)-z_{1}^{0}\left(\bar{\beta}_{1}\right)\right|^{2} d x .
\end{aligned}
$$

From the definition of $\psi$ in 135 and $(126)$, using Sobolev and Cauchy-Schwarz inequalities, we have

$$
\begin{aligned}
& \int_{0}^{\xi_{1}(t ; 0,0)}\left|b_{1} \psi\left(\alpha_{1}\right)-b_{1} \bar{\psi}\left(\bar{\alpha}_{1}\right)\right|^{2} d x \\
= & \int_{0}^{\xi_{1}(t ; 0,0)} b_{1}^{2}\left|\int_{0}^{\alpha_{1}} \frac{v_{1}\left(s, x_{0}\right)+v_{2}\left(s, x_{0}\right)}{2} d s-\int_{0}^{\bar{\alpha}_{1}} \frac{\bar{v}_{1}\left(s, x_{0}\right)+\bar{v}_{2}\left(s, x_{0}\right)}{2} d s\right|^{2} d x \\
\leqslant & C\left|\mathbf{v}\left(\cdot, x_{0}\right)-\overline{\mathbf{v}}\left(\cdot, x_{0}\right)\right|_{L^{2}\left((0, T) ; \mathbb{R}^{2}\right)}^{2}+C\left|\overline{\mathbf{v}}\left(\cdot, x_{0}\right)\right|_{H^{2}\left((0, T) ; \mathbb{R}^{2}\right)}^{2} \int_{0}^{\xi_{1}(t ; 0,0)}\left|\alpha_{1}-\bar{\alpha}_{1}\right|^{2} d x .
\end{aligned}
$$

By the definition of $\gamma_{1}$ in $(140)$ and the corresponding definition of $\bar{\gamma}_{1}$ and using 142 , we obtain

$$
\int_{0}^{\xi_{1}(t ; 0,0)}\left|k_{1} z_{1}^{0}\left(\gamma_{1}\right)-k_{1} z_{1}^{0}\left(\bar{\gamma}_{1}\right)\right|^{2} d x \leqslant C\left|z_{1}^{0}\right|_{H^{2}\left(\left(0, x_{0}\right) ; \mathbb{R}\right)}^{2} \int_{0}^{\xi_{1}(t ; 0,0)}\left|\alpha_{1}-\bar{\alpha}_{1}\right|^{2} d x
$$


Combining 170-172, we get

$$
\begin{aligned}
& \int_{0}^{x_{0}}\left|z_{1}(t, x)-\bar{z}_{1}(t, x)\right|^{2} d x \\
\leqslant & C\left(\left|z_{1}^{0}\right|_{H^{2}\left(\left(0, x_{0}\right) ; \mathbb{R}\right)}^{2}+\left|\overline{\mathbf{v}}\left(\cdot, x_{0}\right)\right|_{H^{2}\left((0, T) ; \mathbb{R}^{2}\right)}^{2}\right) \int_{0}^{\xi_{1}(t ; 0,0)}\left|\alpha_{1}-\bar{\alpha}_{1}\right|^{2} d x \\
& +\left|z_{1}^{0}\right|_{H^{2}\left(\left(0, x_{0}\right) ; \mathbb{R}\right)}^{2} \int_{\bar{\xi}_{1}(t ; 0,0)}^{x_{0}}\left|\beta_{1}-\bar{\beta}_{1}\right|^{2} d x+\int_{\xi_{1}(t ; 0,0)}^{\bar{\xi}_{1}(t ; 0,0)}\left|z_{1}^{0}\left(\beta_{1}\right)-\left(k_{1} z_{1}^{0}\left(\bar{\gamma}_{1}\right)+b_{1} \bar{\psi}\left(\bar{\alpha}_{1}\right)\right)\right|^{2} d x \\
& +C\left|\mathbf{v}\left(\cdot, x_{0}\right)-\overline{\mathbf{v}}\left(\cdot, x_{0}\right)\right|_{L^{2}\left((0, T) ; \mathbb{R}^{2}\right)}^{2} .
\end{aligned}
$$

We estimate each term in 173 separately. By the definition of $\beta_{1}$ in 138 and the corresponding definition of $\bar{\beta}_{1}$, we have

$$
\int_{\bar{\xi}_{1}(t ; 0,0)}^{x_{0}}\left|\beta_{1}-\bar{\beta}_{1}\right|^{2} d x=\int_{\bar{\xi}_{1}(t ; 0,0)}^{x_{0}}\left|\xi_{1}(0 ; t, x)-\bar{\xi}_{1}(0 ; t, x)\right|^{2} d x .
$$

Now, let us estimate $\left|\xi_{1}(0 ; t, x)-\bar{\xi}_{1}(0 ; t, x)\right|$. From the definition of $x_{s}$ in 126$)$ and the definitions of $\xi_{1}$ and $\bar{\xi}_{1}$, see $(137)$, we get for any $s \in[0, t]$ that

$$
\begin{aligned}
& \left|\xi_{1}(s ; t, x)-\bar{\xi}_{1}(s ; t, x)\right| \\
= & \left|\int_{s}^{t} f_{1}\left(\theta, \xi_{1}(\theta ; t, x)\right) d \theta-\int_{s}^{t} \bar{f}_{1}\left(\theta, \bar{\xi}_{1}(\theta ; t, x)\right) d \theta\right| \\
\leqslant & \int_{s}^{t}\left(\left|\left(1+v_{1}\left(\theta, \xi_{1}\right)-\xi_{1} \frac{v_{1}\left(\theta, x_{0}\right)+v_{2}\left(\theta, x_{0}\right)}{2 x_{0}}\right) \frac{x_{0}}{x_{s}\left(\mathbf{v}\left(\cdot, x_{0}\right)\right)(\theta) x_{s}\left(\overline{\mathbf{v}}\left(\cdot, x_{0}\right)\right)(\theta)}\right|\right. \\
& \left.+\int_{s}^{\theta}\left|\frac{v_{1}\left(\alpha, x_{0}\right)-\bar{v}_{1}\left(\alpha, x_{0}\right)+v_{2}\left(\alpha, x_{0}\right)-\bar{v}_{2}\left(\alpha, x_{0}\right)}{2}\right| d \alpha\right) d \theta \\
\leqslant & C\left|\mathbf{v}\left(\cdot, x_{0}\right)-\overline{\mathbf{v}}\left(\cdot, x_{0}\right)\right|_{L^{2}\left((0, T) ; \mathbb{R}^{2}\right)}+C \nu \int_{s}^{t}\left|\xi_{1}(\theta ; t, x)-\bar{\xi}_{1}(\theta ; t, x)\right| d \theta \\
& +C \int_{s}^{t}\left|v_{1}\left(\theta, \bar{\xi}_{1}(\theta ; t, x)\right)-\bar{v}_{1}\left(\theta, \bar{\xi}_{1}(\theta ; t, x)\right)\right| d \theta .
\end{aligned}
$$

From 175$)$, we get for $\nu \in\left(0, \nu_{0}\right]$ sufficiently small and for $\bar{\xi}_{1}(t ; 0,0)<x \leqslant x_{0}$ that

$$
\begin{aligned}
\left|\xi_{1}(\cdot ; t, x)-\bar{\xi}_{1}(\cdot ; t, x)\right|_{C^{0}([0, t] ; \mathbb{R})} \leqslant & C\left|\mathbf{v}\left(\cdot, x_{0}\right)-\overline{\mathbf{v}}\left(\cdot, x_{0}\right)\right|_{L^{2}\left((0, T) ; \mathbb{R}^{2}\right)} \\
& +C \int_{0}^{t}\left|v_{1}\left(\theta, \bar{\xi}_{1}(\theta ; t, x)\right)-\bar{v}_{1}\left(\theta, \bar{\xi}_{1}(\theta ; t, x)\right)\right| d \theta
\end{aligned}
$$

Thus, from 174 and 176 we have

$$
\begin{aligned}
\int_{\bar{\xi}_{1}(t ; 0,0)}^{x_{0}}\left|\beta_{1}-\bar{\beta}_{1}\right|^{2} d x \leqslant & C\left|\mathbf{v}\left(\cdot, x_{0}\right)-\overline{\mathbf{v}}\left(\cdot, x_{0}\right)\right|_{L^{2}\left((0, T) ; \mathbb{R}^{2}\right)}^{2} \\
& +C \int_{\bar{\xi}_{1}(t ; 0,0)}^{x_{0}}\left(\int_{0}^{t}\left|v_{1}\left(\theta, \bar{\xi}_{1}(\theta ; t, x)\right)-\bar{v}_{1}\left(\theta, \bar{\xi}_{1}(\theta ; t, x)\right)\right| d \theta\right)^{2} d x \\
\leqslant & C\left|\mathbf{v}\left(\cdot, x_{0}\right)-\overline{\mathbf{v}}\left(\cdot, x_{0}\right)\right|_{L^{2}\left((0, T) ; \mathbb{R}^{2}\right)}^{2} \\
& +C \int_{0}^{t} \int_{\bar{\xi}_{1}(t ; 0,0)}^{x_{0}}\left|v_{1}\left(\theta, \bar{\xi}_{1}(\theta ; t, x)\right)-\bar{v}_{1}\left(\theta, \bar{\xi}_{1}(\theta ; t, x)\right)\right|^{2} d x d \theta \\
\leqslant & C\left|\mathbf{v}\left(\cdot, x_{0}\right)-\overline{\mathbf{v}}\left(\cdot, x_{0}\right)\right|_{L^{2}\left((0, T) ; \mathbb{R}^{2}\right)}^{2} \\
& +C\left|v_{1}-\bar{v}_{1}\right|_{L^{\infty}\left((0, T) ; L^{2}\left(\left(0, x_{0}\right) ; \mathbb{R}\right)\right)}^{2} .
\end{aligned}
$$

The last inequality is obtained using the change of variable $y=\bar{\xi}_{1}(\theta ; t, x)$, well-defined for $0 \leqslant \theta \leqslant t \leqslant$ $T$ and $\bar{\xi}_{1}(t ; 0,0)<x \leqslant x_{0}$. Let us now estimate $\left|\alpha_{1}-\bar{\alpha}_{1}\right|_{L^{2}\left(\left(0, \xi_{1}(t ; 0,0)\right) ; \mathbb{R}\right)}$. Without loss of generality, 
we may assume that $\alpha_{1} \leqslant \bar{\alpha}_{1}$. By definition of $\alpha_{1}$ in 139 and the corresponding definition of $\bar{\alpha}_{1}$, we have

$$
\int_{\alpha_{1}}^{t} f_{1}\left(s, \xi_{1}(s ; t, x)\right) d s=x=\int_{\bar{\alpha}_{1}}^{t} \bar{f}_{1}\left(s, \bar{\xi}_{1}(s ; t, x)\right) d s .
$$

Hence, similarly to 175 , we get

$$
\begin{aligned}
\left|\alpha_{1}-\bar{\alpha}_{1}\right| \leqslant & \frac{1}{\inf _{(t, x) \in[0, T] \times\left[0, x_{0}\right]}\left|f_{1}(t, x)\right|} \int_{\bar{\alpha}_{1}}^{t}\left|f_{1}\left(s, \xi_{1}(s ; t, x)\right)-\bar{f}_{1}\left(s, \bar{\xi}_{1}(s ; t, x)\right)\right| d s \\
\leqslant & C\left|\mathbf{v}\left(\cdot, x_{0}\right)-\overline{\mathbf{v}}\left(\cdot, x_{0}\right)\right|_{L^{2}\left((0, T) ; \mathbb{R}^{2}\right)}+C \nu \int_{\bar{\alpha}_{1}}^{t}\left|\xi_{1}(\theta ; t, x)-\bar{\xi}_{1}(\theta ; t, x)\right| d \theta \\
& +C \int_{\bar{\alpha}_{1}}^{t}\left|v_{1}\left(\theta, \bar{\xi}_{1}(\theta ; t, x)\right)-\bar{v}_{1}\left(\theta, \bar{\xi}_{1}(\theta ; t, x)\right)\right| d \theta .
\end{aligned}
$$

Similarly to the proof of $[176)$, for $\nu \in\left(0, \nu_{0}\right]$ sufficiently small, we can obtain that (note that $\xi_{1}(s ; t, x)$ and $\bar{\xi}_{1}(s ; t, x)$ for any $s \in\left[\bar{\alpha}_{1}, t\right]$ are well defined as we assume that $\left.\alpha_{1} \leqslant \bar{\alpha}_{1}\right)$

$$
\begin{aligned}
\left|\xi_{1}(\cdot ; t, x)-\bar{\xi}_{1}(\cdot ; t, x)\right|_{C^{0}\left(\left[\bar{\alpha}_{1}, t\right] ; \mathbb{R}\right)} \leqslant & C\left|\mathbf{v}\left(\cdot, x_{0}\right)-\overline{\mathbf{v}}\left(\cdot, x_{0}\right)\right|_{L^{2}\left((0, T) ; \mathbb{R}^{2}\right)} \\
& +C \int_{\bar{\alpha}}^{t}\left|v_{1}\left(\theta, \bar{\xi}_{1}(\theta ; t, x)\right)-\bar{v}_{1}\left(\theta, \bar{\xi}_{1}(\theta ; t, x)\right)\right| d \theta .
\end{aligned}
$$

Using this inequality in (179) and performing similarly as in (177), we can obtain

$$
\int_{0}^{\xi_{1}(t ; 0,0)}\left|\alpha_{1}-\bar{\alpha}_{1}\right|^{2} d x \leqslant C\left|\mathbf{v}\left(\cdot, x_{0}\right)-\overline{\mathbf{v}}\left(\cdot, x_{0}\right)\right|_{L^{2}\left((0, T) ; \mathbb{R}^{2}\right)}^{2}+C\left|v_{1}-\bar{v}_{1}\right|_{L^{\infty}\left((0, T) ; L^{2}\left(\left(0, x_{0}\right) ; \mathbb{R}\right)\right)}^{2} .
$$

Let us now focus on the estimation of the term $\int_{\xi_{1}(t ; 0,0)}^{\bar{\xi}_{1}(t ; 0,0)}\left|z_{1}^{0}\left(\beta_{1}\right)-\left(k_{1} z_{1}^{0}\left(\bar{\gamma}_{1}\right)+b_{1} \bar{\psi}\left(\bar{\alpha}_{1}\right)\right)\right|^{2} d x$ in 173 . Using the compatibility condition 25, we have

$$
\begin{aligned}
& \int_{\xi_{1}(t ; 0,0)}^{\bar{\xi}_{1}(t ; 0,0)}\left|z_{1}^{0}\left(\beta_{1}\right)-\left(k_{1} z_{1}^{0}\left(\bar{\gamma}_{1}\right)+b_{1} \bar{\psi}\left(\bar{\alpha}_{1}\right)\right)\right|^{2} d x \\
= & \int_{\xi_{1}(t ; 0,0)}^{\bar{\xi}_{1}(t ; 0,0)}\left|z_{1}^{0}\left(\beta_{1}\right)-z_{1}^{0}(0)+z_{1}^{0}(0)-\left(k_{1} z_{1}^{0}\left(\bar{\gamma}_{1}\right)+b_{1} \bar{\psi}\left(\bar{\alpha}_{1}\right)\right)\right|^{2} d x \\
= & \int_{\xi_{1}(t ; 0,0)}^{\bar{\xi}_{1}(t ; 0,0)}\left|z_{1}^{0}\left(\beta_{1}\right)-z_{1}^{0}(0)+k_{1} z_{1}^{0}\left(x_{0}\right)+b_{1}\left(x_{0}-x_{s 0}\right)-\left(k_{1} z_{1}^{0}\left(\bar{\gamma}_{1}\right)+b_{1} \bar{\psi}\left(\bar{\alpha}_{1}\right)\right)\right|^{2} d x \\
\leqslant & C\left|z_{1}^{0}\right|_{H^{2}\left(\left(0, x_{0}\right) ; \mathbb{R}\right)}^{2} \int_{\xi_{1}(t ; 0,0)}^{\bar{\xi}_{1}(t ; 0,0)}\left|\beta_{1}\right|^{2} d x+C\left|z_{1}^{0}\right|_{H^{2}\left(\left(0, x_{0}\right) ; \mathbb{R}\right)}^{2} \int_{\xi_{1}(t ; 0,0)}^{\bar{\xi}_{1}(t ; 0,0)}\left|x_{0}-\bar{\gamma}_{1}\right|^{2} d x \\
& +C \int_{\xi_{1}(t ; 0,0)}^{\bar{\xi}_{1}(t ; 0,0)}\left|\int_{0}^{\bar{\alpha}_{1}} \frac{\bar{v}_{1}\left(s, x_{0}\right)+\bar{v}_{2}\left(s, x_{0}\right)}{2} d s\right|^{2} d x .
\end{aligned}
$$

We first estimate $\int_{\xi_{1}(t ; 0,0)}^{\bar{\xi}_{1}(t ; 0,0)}\left|\beta_{1}\right|^{2} d x$. As $\xi_{1}(s ; t, x)$ is increasing with respect to $s \in[0, t]$, we have

$$
\left|\beta_{1}\right|<\left|\xi_{1}\left(\bar{\alpha}_{1} ; t, x\right)\right|=\left|\xi_{1}\left(\bar{\alpha}_{1} ; t, x\right)-\bar{\xi}_{1}\left(\bar{\alpha}_{1} ; t, x\right)\right| \leqslant\left|\xi_{1}(\cdot ; t, x)-\bar{\xi}_{1}(\cdot ; t, x)\right|_{C^{0}\left(\left[\bar{\alpha}_{1}, t\right] ; \mathbb{R}\right)},
$$

then by 180 and performing the same proof as in 177 , we get

$$
\int_{\xi_{1}(t ; 0,0)}^{\bar{\xi}_{1}(t ; 0,0)}\left|\beta_{1}\right|^{2} d x \leqslant C\left|\mathbf{v}\left(\cdot, x_{0}\right)-\overline{\mathbf{v}}\left(\cdot, x_{0}\right)\right|_{L^{2}\left((0, T) ; \mathbb{R}^{2}\right)}^{2}+C\left|v_{1}-\bar{v}_{1}\right|_{L^{\infty}\left((0, T) ; L^{2}\left(\left(0, x_{0}\right) ; \mathbb{R}\right)\right)}^{2} .
$$

Let us now look at the second term in $(182)$, from 1142$)$ and the definition of $\bar{\gamma}_{1}$, we have

$$
\int_{\xi_{1}(t ; 0,0)}^{\bar{\xi}_{1}(t ; 0,0)}\left|x_{0}-\bar{\gamma}_{1}\right|^{2} d x=\int_{\xi_{1}(t ; 0,0)}^{\bar{\xi}_{1}(t ; 0,0)}\left|\bar{\xi}_{1}\left(0 ; 0, x_{0}\right)-\bar{\xi}_{1}\left(0 ; \bar{\alpha}_{1}, x_{0}\right)\right|^{2} d x
$$




$$
\leqslant\left|\partial_{t} \bar{\xi}_{1}\right|_{0}^{2} \int_{\xi_{1}(t ; 0,0)}^{\bar{\xi}_{1}(t ; 0,0)}\left|\bar{\alpha}_{1}\right|^{2} d x \leqslant C \int_{\xi_{1}(t ; 0,0)}^{\bar{\xi}_{1}(t ; 0,0)}\left|\bar{\alpha}_{1}\right|^{2} d x
$$

It is easy to deal with the last term in 182$)$, one has

$$
\int_{\xi_{1}(t ; 0,0)}^{\bar{\xi}_{1}(t ; 0,0)}\left|\int_{0}^{\bar{\alpha}_{1}} \frac{\bar{v}_{1}\left(s, x_{0}\right)+\bar{v}_{2}\left(s, x_{0}\right)}{2} d s\right|^{2} d x \leqslant C\left|\overline{\mathbf{v}}\left(\cdot, x_{0}\right)\right|_{H^{2}\left((0, T) ; \mathbb{R}^{2}\right)}^{2} \int_{\xi_{1}(t ; 0,0)}^{\bar{\xi}_{1}(t ; 0,0)}\left|\bar{\alpha}_{1}\right|^{2} d x .
$$

Thus, we only have to estimate $\int_{\xi_{1}(t ; 0,0)}^{\bar{\xi}_{1}(t ; 0,0)}\left|\bar{\alpha}_{1}\right|^{2} d x$. Noticing that for any fixed $(t, x)$, the characteristic $\bar{\xi}_{1}(s ; t, x)$ is increasing with respect to $s \in\left[\bar{\alpha}_{1}, t\right]$ and that $\xi_{1}^{-1}(\cdot ; t, x)\left(\beta_{1}\right)=0$, we obtain

$$
\bar{\alpha}_{1}<\bar{\xi}_{1}^{-1}(\cdot ; t, x)\left(\beta_{1}\right)-\xi_{1}^{-1}(\cdot ; t, x)\left(\beta_{1}\right) .
$$

Moreover,

$$
\begin{aligned}
& \beta_{1}=x+\int_{t}^{\xi_{1}^{-1}(\cdot ; t, x)\left(\beta_{1}\right)} f_{1}\left(s ; \xi_{1}(s ; t, x)\right) d \theta, \\
& \beta_{1}=x+\int_{t}^{\bar{\xi}_{1}^{-1}(\cdot ; t, x)\left(\beta_{1}\right)} \bar{f}_{1}\left(s ; \bar{\xi}_{1}(s ; t, x)\right) d \theta .
\end{aligned}
$$

Then similarly as for 179 , we can prove that

$$
\begin{aligned}
\left|\bar{\xi}_{1}^{-1}(\cdot ; t, x)\left(\beta_{1}\right)-\xi_{1}^{-1}(\cdot ; t, x)\left(\beta_{1}\right)\right| \leqslant & C\left|\mathbf{v}\left(\cdot, x_{0}\right)-\overline{\mathbf{v}}\left(\cdot, x_{0}\right)\right|_{L^{2}(0, T)} \\
& +C \nu \int_{\bar{\xi}_{1}^{-1}(\cdot ; t, x)\left(\beta_{1}\right)}^{t}\left|\xi_{1}(\theta ; t, x)-\bar{\xi}_{1}(\theta ; t, x)\right| d \theta \\
& +C \int_{\bar{\xi}_{1}^{-1}(\cdot ; t, x)\left(\beta_{1}\right)}^{t}\left|v_{1}\left(\theta, \bar{\xi}_{1}(\theta ; t, x)\right)-\bar{v}_{1}\left(\theta, \bar{\xi}_{1}(\theta ; t, x)\right)\right| d \theta .
\end{aligned}
$$

Thus, similarly as in the proof for (181), we get

$$
\begin{aligned}
\int_{\xi_{1}(t ; 0,0)}^{\bar{\xi}_{1}(t ; 0,0)}\left|\bar{\alpha}_{1}\right|^{2} d x \leqslant & C\left|\mathbf{v}\left(\cdot, x_{0}\right)-\overline{\mathbf{v}}\left(\cdot, x_{0}\right)\right|_{L^{2}\left((0, T) ; \mathbb{R}^{2}\right)}^{2} \\
& +C\left|v_{1}-\bar{v}_{1}\right|_{L^{\infty}\left((0, T) ; L^{2}\left(\left(0, x_{0}\right) ; \mathbb{R}\right)\right)}^{2} .
\end{aligned}
$$

Finally, using estimations (184) and 185)-(187), 182) becomes

$$
\begin{aligned}
& \int_{\xi_{1}(t ; 0,0)}^{\bar{\xi}_{1}(t ; 0,0)}\left|z_{1}^{0}\left(\beta_{1}\right)-\left(k_{1} z_{1}^{0}\left(\bar{\gamma}_{1}\right)+b_{1} \psi\left(\bar{\alpha}_{1}\right)\right)\right|^{2} d x \\
& \leqslant C\left(\left|z_{1}^{0}\right|_{H^{2}\left(\left(0, x_{0}\right) ; \mathbb{R}\right)}^{2}+\left|\overline{\mathbf{v}}\left(\cdot, x_{0}\right)\right|_{H^{2}\left((0, T) ; \mathbb{R}^{2}\right)}^{2}\right)\left(\left|\mathbf{v}\left(\cdot, x_{0}\right)-\overline{\mathbf{v}}\left(\cdot, x_{0}\right)\right|_{L^{2}\left((0, T) ; \mathbb{R}^{2}\right)}^{2}\right. \\
& \left.+\left|v_{1}-\bar{v}_{1}\right|_{L^{\infty}\left((0, T) ; L^{2}\left(\left(0, x_{0}\right) ; \mathbb{R}\right)\right)}^{2}\right) .
\end{aligned}
$$

Combining (173), 177), 181) and (188), we get

$$
\begin{aligned}
& \quad \begin{array}{l}
\left|z_{1}-\bar{z}_{1}\right|_{L^{\infty}\left((0, T) ; L^{2}\left(\left(0, x_{0}\right) ; \mathbb{R}\right)\right)}^{2} \\
\leqslant C\left(\left|z_{1}^{0}\right|_{H^{2}\left(\left(0, x_{0}\right) ; \mathbb{R}\right)}^{2}+\left|\overline{\mathbf{v}}\left(\cdot, x_{0}\right)\right|_{H^{2}\left((0, T) ; \mathbb{R}^{2}\right)}^{2}\right)\left(\left|\mathbf{v}\left(\cdot, x_{0}\right)-\overline{\mathbf{v}}\left(\cdot, x_{0}\right)\right|_{L^{2}\left((0, T) ; \mathbb{R}^{2}\right)}^{2}+\left|v_{1}-\bar{v}_{1}\right|_{\left.L^{\infty}\left((0, T) ; L^{2}\left(\left(0, x_{0}\right) ; \mathbb{R}\right)\right)\right)}^{2}\right. \\
+C\left|\mathbf{v}\left(\cdot, x_{0}\right)-\overline{\mathbf{v}}\left(\cdot, x_{0}\right)\right|_{L^{2}\left((0, T) ; \mathbb{R}^{2}\right) \cdot}^{2} \quad(189)
\end{array}
\end{aligned}
$$

We are left with estimating $\left|\mathbf{z}\left(\cdot, x_{0}\right)-\overline{\mathbf{z}}\left(\cdot, x_{0}\right)\right|_{L^{2}\left((0, T) ; \mathbb{R}^{2}\right)}$ in order to obtain 169$)$. Here we give the estimation for $z_{1}$. Using (176), we get

$$
\int_{0}^{T}\left|z_{1}\left(t, x_{0}\right)-\bar{z}_{1}\left(t, x_{0}\right)\right|^{2} d t
$$




$$
\begin{aligned}
= & \int_{0}^{T}\left|z_{1}^{0}\left(\xi_{1}\left(0 ; t, x_{0}\right)\right)-z_{1}^{0}\left(\bar{\xi}_{1}\left(0 ; t, x_{0}\right)\right)\right|^{2} \\
\leqslant & \left|z_{1 x}^{0}\right|_{0}^{2} \int_{0}^{T}\left|\xi_{1}\left(0 ; t, x_{0}\right)-\bar{\xi}_{1}\left(0 ; t, x_{0}\right)\right|^{2} d t \\
\leqslant & C\left|z_{1}^{0}\right|_{H^{2}\left(\left(0, x_{0}\right) ; \mathbb{R}\right)}^{2}\left|\mathbf{v}\left(\cdot, x_{0}\right)-\overline{\mathbf{v}}\left(\cdot, x_{0}\right)\right|_{L^{2}\left((0, T) ; \mathbb{R}^{2}\right)}^{2} \\
& +C\left|z_{1}^{0}\right|_{H^{2}\left(\left(0, x_{0}\right) ; \mathbb{R}\right)}^{2} \int_{0}^{T} \int_{0}^{t}\left|v_{1}\left(\theta, \bar{\xi}_{1}\left(\theta ; t, x_{0}\right)\right)-\bar{v}_{1}\left(\theta, \bar{\xi}_{1}\left(\theta ; t, x_{0}\right)\right)\right|^{2} d \theta d t \\
\leqslant & C\left|z_{1}^{0}\right|_{H^{2}\left(\left(0, x_{0}\right) ; \mathbb{R}\right)}^{2}\left|\mathbf{v}\left(\cdot, x_{0}\right)-\overline{\mathbf{v}}\left(\cdot, x_{0}\right)\right|_{L^{2}\left((0, T) ; \mathbb{R}^{2}\right)}^{2} \\
& +C\left|z_{1}^{0}\right|_{H^{2}\left(\left(0, x_{0}\right) ; \mathbb{R}\right)}^{2} \int_{0}^{T} \int_{\theta}^{T}\left|v_{1}\left(\theta, \bar{\xi}_{1}\left(\theta ; t, x_{0}\right)\right)-\bar{v}_{1}\left(\theta, \bar{\xi}_{1}\left(\theta ; t, x_{0}\right)\right)\right|^{2} d t d \theta \\
\leqslant & C\left|z_{1}^{0}\right|_{H^{2}\left(\left(0, x_{0}\right) ; \mathbb{R}\right)}^{2}\left(\left|\mathbf{v}\left(\cdot, x_{0}\right)-\overline{\mathbf{v}}\left(\cdot, x_{0}\right)\right|_{L^{2}\left((0, T) ; \mathbb{R}^{2}\right)}^{2}+\left|v_{1}-\bar{v}_{1}\right|_{\left.L^{\infty}\left((0, T) ; L^{2}\left(\left(0, x_{0}\right) ; \mathbb{R}\right)\right)\right)}^{2} .\right.
\end{aligned}
$$

The last inequality is obtained by changing the variable $y=\bar{\xi}_{1}\left(\theta ; t, x_{0}\right)$. Similar estimates can be done for $z_{2}$. Hence, from $(189)$ and $(190)$, there exists $M>0$ such that for $\delta$ sufficiently small and $\nu \in\left(0, \nu_{2}\right]$, where $\nu_{2} \in\left(0, \nu_{1}\right]$ is sufficiently small and depends only on $C$, we have

$$
\begin{aligned}
& |\mathbf{z}-\overline{\mathbf{z}}|_{L^{\infty}\left((0, T) ; L^{2}\left(\left(0, x_{0}\right) ; \mathbb{R}^{2}\right)\right)}+M\left|\mathbf{z}\left(\cdot, x_{0}\right)-\overline{\mathbf{z}}\left(\cdot, x_{0}\right)\right|_{L^{2}\left((0, T) ; \mathbb{R}^{2}\right)} \\
\leqslant & \frac{1}{2}|\mathbf{v}-\overline{\mathbf{v}}|_{L^{\infty}\left((0, T) ; L^{2}\left(\left(0, x_{0}\right) ; \mathbb{R}^{2}\right)\right)}+\frac{M}{2}\left|\mathbf{v}\left(\cdot, x_{0}\right)-\overline{\mathbf{v}}\left(\cdot, x_{0}\right)\right|_{L^{2}\left((0, T) ; \mathbb{R}^{2}\right)} .
\end{aligned}
$$

Hence $\mathcal{F}$ is a contraction mapping and has a fixed point $\mathbf{z} \in C_{\nu}\left(\mathbf{z}^{0}, x_{s 0}\right)$, i.e., there exists a unique solution $\mathbf{z} \in C_{\nu}\left(\mathbf{z}^{0}, x_{s 0}\right)$ to the system $(21)-(23)$. Noticing $(126)$, we get that $x_{s} \in C^{1}([0, T] ; \mathbb{R})$. To get the extra regularity $\mathbf{z} \in C^{0}\left([0, T] ; H^{2}\left(\left(0, x_{0}\right) ; \mathbb{R}^{2}\right)\right)$, we adapt the proof given by Majda [33, p.4446]. There, the author used energy estimates method for an initial value problem. Using this method for our boundary value problem, we have to be careful with the boundary terms when integrating by parts. Substituting $\mathbf{v}$ by $\mathbf{z}$ in $\psi(t)$ and $f_{1}(t, x)$ in the expression of $z_{1 x}, z_{1 x x}$ in (158) and (159), noticing (120)-122 and computing similar estimates as in 164) and 165), we can obtain the "hidden" regularity $\mathbf{z}_{x}\left(\cdot, x_{0}\right) \in L^{2}\left((0, T) ; \mathbb{R}^{2}\right)$ and $\mathbf{z}_{x x}\left(\cdot, x_{0}\right) \in L^{2}\left((0, T) ; \mathbb{R}^{2}\right)$ together with estimates on $\left|\mathbf{z}_{x}\left(\cdot, x_{0}\right)\right|_{L^{2}\left((0, T) ; \mathbb{R}^{2}\right)}$ and $\left|\mathbf{z}_{x x}\left(\cdot, x_{0}\right)\right|_{L^{2}\left((0, T) ; \mathbb{R}^{2}\right)}$, which are sufficient to take care of the boundary terms when integrating by parts. This concludes the proof of the existence and uniqueness of a classical solution $x_{s}(t) \in C^{1}([0, T] ; \mathbb{R})$ and $\mathbf{z} \in C^{0}\left([0, T] ; H^{2}\left(\left(0, x_{0}\right) ; \mathbb{R}^{2}\right)\right)$ in $C_{\nu}\left(\mathbf{z}^{0}, x_{s 0}\right)$ to the system $\left.(21)-23\right)$ for $T$ satisfying (119).

The estimate (28) for $|\mathbf{z}(t, \cdot)|_{H^{2}\left(\left(0, x_{0}\right) ; \mathbb{R}^{2}\right)}$ part can be obtained from estimates (166)-(167) by firstly replacing $\mathbf{v}$ with $\mathbf{z}$ and then applying (155)-(156). Noticing the definition of $x_{s}$ in (126) and applying (155)-156 again, the estimate for the $\left|x_{s}(t)-x_{0}\right|$ part follows.

Next, we show the uniqueness of the solution in $C^{0}\left([0, T] ; H^{2}\left(\left(0, x_{0}\right) ; \mathbb{R}^{2}\right)\right)$. Suppose that there is another solution $\tilde{\mathbf{z}} \in C^{0}\left([0, T] ; H^{2}\left(\left(0, x_{0}\right) ; \mathbb{R}^{2}\right)\right)$, we prove that $\tilde{\mathbf{z}} \in C_{\nu}\left(\mathbf{z}^{0}, x_{s 0}\right)$, for $\delta$ sufficiently small. To that end, assume that $\mathbf{z}(t, \cdot)=\tilde{\mathbf{z}}(t, \cdot)$ for any $t \in[0, \tau]$ with $\tau \in[0, T]$. If $\tau \neq T$, by [28), for $\delta$ sufficiently small and as $\tilde{\mathbf{z}} \in C^{0}\left([0, T] ; H^{2}\left(\left(0, x_{0}\right) ; \mathbb{R}^{2}\right)\right)$, one can choose $\tau^{\prime} \in(\tau, T)$ small enough such that $\tilde{\mathbf{z}} \in C_{\nu}\left(\mathbf{z}(\tau), x_{s}(\tau)\right)$ with $T$ is replaced by $\tau^{\prime}-\tau$ and by considering $\tau$ as the new initial time. Thus, $\mathbf{z}(t, \cdot)=\tilde{\mathbf{z}}(t, \cdot)$ for any $t \in\left[0, \tau^{\prime}\right]$. As $|\tilde{\mathbf{z}}(t, \cdot)|_{H^{2}\left(\left(0, x_{0}\right) ; \mathbb{R}^{2}\right)}$ is uniformly continuous on $[0, T]$, and as, moreover $C$ and $\nu$ do not depend on $T$, we can repeat this process and finally get $\mathbf{z}(t, \cdot)=\tilde{\mathbf{z}}(t, \cdot)$ on $[0, T]$.

For general $T>0$, one just needs to take $T_{1}$ satisfying $(119)$ and, noticing that $C$ and $\nu$ do not depend on $T_{1}$, one can apply the above procedure at most $\left[T / T_{1}\right]+1$ times. This concludes the proof of Lemma 3.1 .

Proof of Lemma A.1. From 137, we have

$$
\left\{\begin{array}{l}
\frac{\partial^{2} \xi_{1}(s ; t, x)}{\partial s \partial x}=f_{1 x} \frac{\partial \xi_{1}(s ; t, x)}{\partial x} \\
\frac{\partial \xi_{1}(t ; t, x)}{\partial x}=1
\end{array}\right.
$$




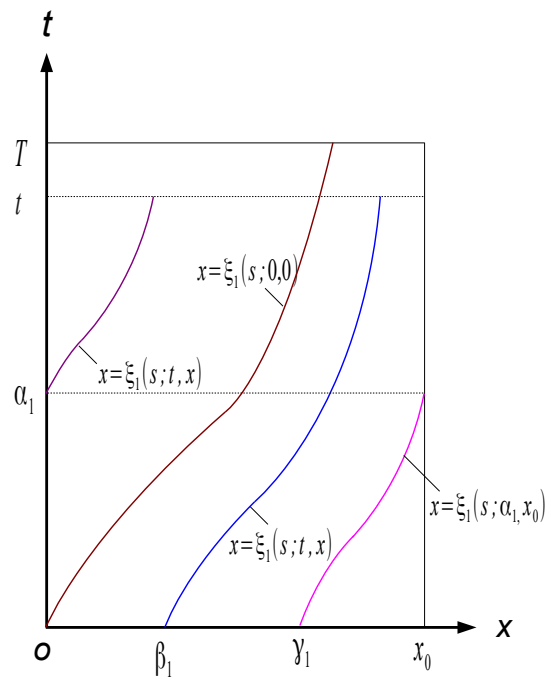

Figure 2: Demonstration of the characteristics.

and

Thus,

$$
\left\{\begin{array}{l}
\frac{\partial^{2} \xi_{1}(s ; t, x)}{\partial s \partial t}=f_{1 x} \frac{\partial \xi_{1}(s ; t, x)}{\partial t} \\
\frac{\partial \xi_{1}(s ; s, x)}{\partial s}+\frac{\partial \xi_{1}(s ; s, x)}{\partial t}=0
\end{array}\right.
$$

$$
\begin{gathered}
\partial_{x} \xi_{1}(s ; t, x)=e^{-\int_{s}^{t} f_{1 x}\left(\theta, \xi_{1}(\theta ; t, x)\right) d \theta}, \\
\partial_{t} \xi_{1}(s ; t, x)=-f_{1}(t, x) e^{-\int_{s}^{t} f_{1 x}\left(\theta, \xi_{1}(\theta ; t, x)\right) d \theta} .
\end{gathered}
$$

From 194-195 and noticing $\beta_{1}=\xi_{1}(0 ; t, x)$, we have

$$
\frac{\partial \beta_{1}}{\partial t}=-f_{1}(t, x) e^{-\int_{0}^{t} f_{1 x}\left(\theta, \xi_{1}(\theta ; t, x)\right) d \theta}, \quad \frac{\partial \beta_{1}}{\partial x}=e^{-\int_{0}^{t} f_{1 x}\left(\theta, \xi_{1}(\theta ; t, x)\right) d \theta} .
$$

From 194, noticing $\xi_{1}\left(\alpha_{1} ; t, x\right)=0$ and by chain rules, we have

$$
\frac{\partial \alpha_{1}}{\partial x}=-\frac{1}{f_{1}\left(\alpha_{1}, 0\right)} e^{-\int_{\alpha_{1}}^{t} f_{1 x}\left(s, \xi_{1}(s ; t, x)\right) d s},
$$

and as $\gamma_{1}=\xi_{1}\left(0 ; \alpha_{1}, x_{0}\right)$, we obtain from that

$$
\frac{\partial \gamma_{1}}{\partial x}=\frac{d \gamma_{1}}{d \alpha_{1}} \frac{\partial \alpha_{1}}{\partial x}=\frac{f_{1}\left(\alpha_{1}, x_{0}\right)}{f_{1}\left(\alpha_{1}, 0\right)} e^{-\int_{0}^{\alpha_{1}} f_{1 x}\left(s, \xi_{1}\left(s ; \alpha_{1}, x_{0}\right)\right) d s-\int_{\alpha_{1}}^{t} f_{1 x}\left(s, \xi_{1}(s ; t, x)\right) d s} .
$$

Observe that for a.e. $s \in(0, T)$ and $x \in\left[0, x_{0}\right]$,

$$
\left|v_{1}(s, x)\right| \leqslant\left|\int_{\theta}^{x} v_{1 x}(s, l) d l\right|+\left|v_{1}(s, \theta)\right|, \forall \theta \in\left[0, x_{0}\right]
$$

and as $v_{1}$ is $H^{1}$ in $x$ and its $L^{2}$-norm is bounded by $\nu$, there exists $\theta$ such that $\left|v_{1}(s, \theta)\right| \leqslant \nu / \sqrt{x_{0}}$, therefore

$$
\left|v_{1}(s, x)\right| \leqslant C \nu
$$

and similarly as $v_{1}$ is $H^{2}$ in $x$ with the same bound and $v_{1 t}$ is in $L^{\infty}\left((0, T) ; H^{1}\left(\left(0, x_{0}\right) ; \mathbb{R}\right)\right)$ with bound $\nu$ from 121

$$
\begin{aligned}
& x \in\left[0, x_{0}\right],\left|v_{1 x}(s, x)\right| \leqslant C \nu, \text { for a.e. } s \in(0, T), \\
& x \in\left[0, x_{0}\right],\left|v_{1 t}(s, x)\right| \leqslant C \nu, \text { for a.e. } s \in(0, T) .
\end{aligned}
$$


From the expression of $f_{1}$ defined in 136 and using $194-(198)$ and $(200-(201)$, after some direct computations, estimates (141)-146 can be obtained. We now demonstrate the estimate (147) in details, while (148)-150 can be treated in a similar way, thus we omit them. From $(196)$, we have

$$
\partial_{t t} \beta_{1}=\left(-f_{1 t}(t, x)+f_{1}(t, x)\left(f_{1 x}(t, x)+\int_{0}^{t} f_{1 x x}\left(\theta, \xi_{1}(\theta ; t, x)\right) \partial_{t} \xi_{1}(\theta ; t, x) d \theta\right)\right) e^{-\int_{0}^{t} f_{1 x}\left(\theta, \xi_{1}(\theta ; t, x)\right) d \theta}
$$

Looking at 136$)$, as $\mathbf{v}$ is only in $L^{\infty}\left((0, T) ; H^{2}\left(\left(0, x_{0}\right) ; \mathbb{R}^{2}\right)\right) \cap W^{1, \infty}\left((0, T) ; H^{1}\left(\left(0, x_{0}\right) ; \mathbb{R}^{2}\right)\right)$

$\cap W^{2, \infty}\left((0, T) ; L^{2}\left(\left(0, x_{0}\right) ; \mathbb{R}^{2}\right)\right)$, this equation is expressed a priori formally in the distribution sense. Thus, we have to be careful when we estimate (147). By (136) and using estimates (141), (142), we get by Cauchy-Schwarz inequality together with the change of variable $y=\xi_{1}\left(\theta ; t, x_{0}\right)$ that

$$
\begin{aligned}
\int_{0}^{T}\left|\partial_{t t} \beta_{1}\left(t, x_{0}\right)\right|^{2} d t & \leqslant C \nu+C \int_{0}^{T}\left|\int_{0}^{t} f_{1 x x}\left(\theta, \xi_{1}\left(\theta ; t, x_{0}\right)\right) \partial_{t} \xi_{1}\left(\theta ; t, x_{0}\right) d \theta\right|^{2} d t \\
& \leqslant C \nu+C \int_{0}^{T} \int_{0}^{t} v_{1 x x}^{2}\left(\theta, \xi_{1}\left(\theta ; t, x_{0}\right)\right) \partial_{t}^{2} \xi_{1}\left(\theta ; t, x_{0}\right) d \theta d t \\
& =C \nu+C \int_{0}^{T} \int_{\theta}^{T} v_{1 x x}^{2}\left(\theta, \xi_{1}\left(\theta ; t, x_{0}\right)\right) \partial_{t}^{2} \xi_{1}\left(\theta ; t, x_{0}\right) d t d \theta \\
& \leqslant C \nu+C \int_{0}^{T} \int_{0}^{x_{0}} v_{1 x x}^{2}(\theta, y) d y d \theta \\
& \leqslant C \nu
\end{aligned}
$$

\section{B Proof of Theorem 5.1}

First observe that, after the change of variables $(19),(20)$, the new equations are

$$
\begin{aligned}
& z_{1 t}+\left(f^{\prime}(1)+\left(f^{\prime}\left(z_{1}+1\right)-f^{\prime}(1)\right)-x \frac{\dot{x}_{s}}{x_{0}}\right) z_{1 x} \frac{x_{0}}{x_{s}}=0, \\
& z_{2 t}+\left(-f^{\prime}(-1)+\left(f^{\prime}(-1)-f^{\prime}\left(z_{2}-1\right)\right)+x \frac{\dot{x}_{s}}{x_{0}}\right) z_{2 x} \frac{x_{0}}{L-x_{s}}=0, \\
& \dot{x}_{s}(t)=\frac{f^{\prime}(1) z_{1}\left(t, x_{0}\right)-f^{\prime}(-1) z_{2}\left(t, x_{0}\right)}{2+\left(z_{1}\left(t, x_{0}\right)-z_{2}\left(t, x_{0}\right)\right)} \\
& +\frac{\left(f\left(z_{1}\left(t, x_{0}\right)+1\right)-f^{\prime}(1) z_{1}\left(t, x_{0}\right)-f(1)\right)-\left(f\left(z_{2}\left(t, x_{0}\right)-1\right)-f^{\prime}(-1) z_{2}\left(t, x_{0}\right)-f(-1)\right)}{2+\left(z_{1}\left(t, x_{0}\right)-z_{2}\left(t, x_{0}\right)\right)}
\end{aligned}
$$

and the boundary conditions remain given by (22). Note that in (203) the expression of $\dot{x}_{s}$ can actually be written as

$$
\dot{x}_{s}(t)=\frac{f^{\prime}(1) z_{1}\left(t, x_{0}\right)-f^{\prime}(-1) z_{2}\left(t, x_{0}\right)}{2}+O\left(\left|\mathbf{z}\left(t, x_{0}\right)\right|^{2}\right) .
$$

Thus, to prove Theorem 5.1. if suffices to show Theorem 4.1 with 203) instead of (21). We still define the Lyapunov function candidate as previously by (31)-(37). Then Lemma 4.1 and Lemma 4.2 remain unchanged. To adapt Lemma 4.3 . one can check that, when differentiating $V_{1}, V_{2}$ and $V_{3}$ along the $C^{3}$ solutions of 203), (22) with associated initial conditions and noticing that under assumption $f(-1)=f(1)$, one has $f^{\prime}(-1) \leqslant 0, f^{\prime}(1) \geqslant 0$ from the property of convex function, we obtain as previously (68), (70) and (74) but with $f^{\prime}(1) p_{1}$ instead of $p_{1}$ and $\left|f^{\prime}(-1)\right| p_{2}$ instead of $p_{2}$ in the boundary terms and $\mu V_{i}$ being replaced by $\mu \min \left(f^{\prime}(1),\left|f^{\prime}(-1)\right|\right) V_{i}$. Then, from (117) and dealing 
with $V_{4}$, we finally get:

$$
\begin{aligned}
\frac{d V_{1}}{d t}+\frac{d V_{4}}{d t} \leqslant & -\mu\left(V_{1}+V_{4}\right) \\
& +\max \left\{\frac{\Theta_{1}}{\varepsilon_{1}}, \frac{\Theta_{2}}{\varepsilon_{2}}\right\} V_{1} \\
& +\left[\frac{x_{0}}{x_{s}} p_{1}\left(k_{1}^{2}-e^{-\frac{\mu x_{0}}{\eta_{1}}}\right) f^{\prime}(1)+\frac{\varepsilon_{1}+\varepsilon_{2}}{8} f^{\prime}(1)^{2}\right] z_{1}^{2}\left(x_{0}\right) \\
& +\left[\frac{x_{0}}{L-x_{s}} p_{2}\left(k_{2}^{2}-e^{-\frac{\mu x_{0}}{\eta_{2}}}\right)\left|f^{\prime}(-1)\right|+\frac{\varepsilon_{1}+\varepsilon_{2}}{8}\left|f^{\prime}(-1)\right|^{2}\right] z_{2}^{2}\left(x_{0}\right) \\
& +f^{\prime}(1)\left[-2 \frac{x_{0}}{x_{s}} p_{1} b_{1} k_{1}-\frac{x_{0}}{x_{s}} \bar{p}_{1}\left(e^{-\frac{\mu x_{0}}{\eta_{1}}}-k_{1}\right)+\kappa\right] z_{1}\left(x_{0}\right)\left(x_{s}-x_{0}\right) \\
& +\left|f^{\prime}(-1)\right|\left[-2 \frac{x_{0}}{L-x_{s}} p_{2} b_{2} k_{2}-\frac{x_{0}}{L-x_{s}} \bar{p}_{2}\left(e^{-\frac{\mu x_{0}}{\eta_{2}}}-k_{2}\right)+\kappa\right] z_{2}\left(x_{0}\right)\left(x_{s}-x_{0}\right) \\
& +\left[\frac{x_{0}}{x_{s}} p_{1} b_{1}^{2} f^{\prime}(1)+\frac{x_{0}}{L-x_{s}} p_{2} b_{2}^{2}\left|f^{\prime}(-1)\right|-\frac{x_{0}}{x_{s}} \bar{p}_{1} b_{1} f^{\prime}(1)-\frac{x_{0}}{L-x_{s}} \bar{p}_{2} b_{2}\left|f^{\prime}(-1)\right|+\mu \kappa\right]\left(x_{s}-x_{0}\right)^{2} \\
& +O\left(\left(|\mathbf{z}|_{H^{2}}+\left|x_{s}-x_{0}\right|\right)^{3}\right),
\end{aligned}
$$

and a similar expression for $V_{2}+V_{5}$ and $V_{3}+V_{6}$ as previously. Thus Lemma 4.3 still holds but with A now defined by

$$
\begin{aligned}
& a_{11}=p_{1}\left(e^{-\frac{\mu x_{0}}{\eta_{1}}}-k_{1}^{2}\right) f^{\prime}(1)-\frac{\varepsilon_{1}+\varepsilon_{2}}{8} f^{\prime}(1)^{2}, \\
& a_{13}=a_{31}=f^{\prime}(1) p_{1} b_{1} k_{1}+f^{\prime}(1) \frac{\bar{p}_{1}}{2}\left(e^{-\frac{\mu x_{0}}{\eta_{1}}}-k_{1}\right)-f^{\prime}(1) \frac{\kappa}{2}, \\
& a_{22}=\frac{x_{0}}{L-x_{0}} p_{2}\left(e^{-\frac{\mu x_{0}}{\eta_{2}}}-k_{2}^{2}\right)\left|f^{\prime}(-1)\right|-\frac{\varepsilon_{1}+\varepsilon_{2}}{8}\left|f^{\prime}(-1)\right|^{2}, \\
& a_{23}=a_{32}=\left|f^{\prime}(-1)\right| \frac{x_{0}}{L-x_{0}} p_{2} b_{2} k_{2}+\left|f^{\prime}(-1)\right| \frac{x_{0}}{L-x_{0}} \frac{\bar{p}_{2}}{2}\left(e^{-\frac{\mu x_{0}}{\eta_{2}}}-k_{2}\right)-\left|f^{\prime}(-1)\right| \frac{\kappa}{2}, \\
& a_{33}=-p_{1} b_{1}^{2} f^{\prime}(1)-\frac{x_{0}}{L-x_{0}} p_{2} b_{2}^{2}\left|f^{\prime}(-1)\right|+\bar{p}_{1} b_{1} f^{\prime}(1)+\frac{x_{0}}{L-x_{0}} \bar{p}_{2} b_{2}\left|f^{\prime}(-1)\right|-\mu \kappa .
\end{aligned}
$$

instead of (88)- 92 . We can then choose $p_{1}, p_{2}, \bar{p}_{1}, \bar{p}_{2}$ as previously by (97)-(99) and $\mathbf{A}$ becomes again diagonal with the expression of its elements given by

$$
\begin{gathered}
a_{33}=\frac{\kappa}{2} f^{\prime}(1) b_{1} e^{\frac{\mu x_{0}}{\eta_{1}}}+\frac{\kappa}{2}\left|f^{\prime}(-1)\right| b_{2} e^{\frac{\mu x_{0}}{\eta_{2}}}-\mu \kappa \\
a_{11}=\frac{\kappa f^{\prime}(1)}{2 b_{1}}\left(1-k_{1}^{2} e^{\frac{\mu x_{0}}{\eta_{1}}}\right)-\frac{\kappa f^{\prime}(1)^{2}}{2 \mu^{2}}\left[b_{1}\left(e^{\frac{\mu x_{0}}{\eta_{1}}}-1\right)+b_{2}\left(e^{\frac{\mu x_{0}}{\eta_{2}}}-1\right)\right], \\
a_{22}=\frac{\kappa\left|f^{\prime}(-1)\right|}{2 b_{2}}\left(1-k_{2}^{2} e^{\frac{\mu x_{0}}{\eta_{2}}}\right)-\frac{\kappa\left|f^{\prime}(-1)\right|^{2}}{2 \mu^{2}}\left[b_{1}\left(e^{\frac{\mu x_{0}}{\eta_{1}}}-1\right)+b_{2}\left(e^{\frac{\mu x_{0}}{\eta_{2}}}-1\right)\right],
\end{gathered}
$$

instead of (102), (105) and (106) respectively. Then to prove Theorem 4.1 with (203) instead of (21), we only need to show now that under assumption (118) there exists $\mu>\gamma$ and $\kappa>1$ such that $a_{i i}>0, i=1,2,3$ and such that (44) holds where $\Theta_{i}, i=1,2$ are still defined by (45). But this can be checked exactly as in the proof of Theorem 4.1. With condition (117), one can now check as in Remark 4 that there always exist parameters $b_{i}$ and $k_{i}$ such that conditions (118) are satisfied.

\section{References}

[1] Fabio Ancona and Andrea Marson. On the attainable set for scalar nonlinear conservation laws with boundary control. SIAM J. Control Optim., 36(1):290-312, 1998.

[2] Georges Bastin and Jean-Michel Coron. On boundary feedback stabilization of non-uniform linear $2 \times 2$ hyperbolic systems over a bounded interval. Systems Control Lett., 60(11):900-906, 2011. 
[3] Georges Bastin and Jean-Michel Coron. Stability and Boundary Stabilisation of 1-D Hyperbolic Systems. Number 88 in Progress in Nonlinear Differential Equations and Their Applications. Springer International, 2016.

[4] Georges Bastin and Jean-Michel Coron. A quadratic Lyapunov function for hyperbolic densityvelocity systems with nonuniform steady states. Systems \& Control Letters, 104:66-71, 2017.

[5] Georges Bastin, Jean-Michel Coron, and Brigitte d'Andréa Novel. On Lyapunov stability of linearised Saint-Venant equations for a sloping channel. Networks and Heterogeneous Media, $4(2): 177-187,2009$.

[6] Sébastien Blandin, Xavier Litrico, Maria Laura Delle Monache, Benedetto Piccoli, and Alexandre Bayen. Regularity and Lyapunov stabilization of weak entropy solutions to scalar conservation laws. IEEE Trans. Automat. Control, 62(4):1620-1635, 2017.

[7] Alberto Bressan and Giuseppe Maria Coclite. On the boundary control of systems of conservation laws. SIAM J. Control Optim., 41(2):607-622, 2002.

[8] Ch. I. Byrnes, D. S. Gilliam, and V. I. Shubov. On the global dynamics of a controlled viscous Burgers' equation. J. Dynam. Control Systems, 4(4):457-519, 1998.

[9] Carlos Castro, Francisco Palacios, and Enrique Zuazua. An alternating descent method for the optimal control of the inviscid Burgers equation in the presence of shocks. Math. Models Methods Appl. Sci., 18(3):369-416, 2008.

[10] Jean-Michel Coron. Control and nonlinearity, volume 136 of Mathematical Surveys and Monographs. American Mathematical Society, Providence, RI, 2007.

[11] Jean-Michel Coron and Georges Bastin. Dissipative boundary conditions for one-dimensional quasi-linear hyperbolic systems: Lyapunov stability for the $C^{1}$-norm. SIAM J. Control Optim., 53(3):1464-1483, 2015.

[12] Jean-Michel Coron, Georges Bastin, and Brigitte d'Andréa Novel. Dissipative boundary conditions for one-dimensional nonlinear hyperbolic systems. SIAM Journal on Control and Optimization, 47(3):1460-1498, 2008.

[13] Jean-Michel Coron, Brigitte d'Andréa Novel, and Georges Bastin. A strict Lyapunov function for boundary control of hyperbolic systems of conservation laws. IEEE Transactions on Automatic Control, 52(1):2-11, 2007.

[14] Jean-Michel Coron, Sylvain Ervedoza, Shyam Sundar Ghoshal, Olivier Glass, and Vincent Perrollaz. Dissipative boundary conditions for $2 \times 2$ hyperbolic systems of conservation laws for entropy solutions in BV. J. Differential Equations, 262(1):1-30, 2017.

[15] Jean-Michel Coron and Hoai-Minh Nguyen. Dissipative boundary conditions for nonlinear 1-d hyperbolic systems: sharp conditions through an approach via time-delay systems. SIAM Journal on Mathematical Analysis, 47(3):2220-2240, 2015.

[16] Jean-Michel Coron, Rafael Vazquez, Miroslav Krstic, and Georges Bastin. Local exponential $H^{2}$ stabilization of a $2 \times 2$ quasilinear hyperbolic system using backstepping. SIAM J. Control Optim., 51(3):2005-2035, 2013.

[17] Jean-Michel Coron and Zhiqiang Wang. Output feedback stabilization for a scalar conservation law with a nonlocal velocity. SIAM J. Math. Anal., 45(5):2646-2665, 2013.

[18] Constantine M. Dafermos. Hyperbolic conservation laws in continuum physics, volume 325 of Grundlehren der Mathematischen Wissenschaften [Fundamental Principles of Mathematical Sciences]. Springer-Verlag, Berlin, third edition, 2010.

[19] James M. Greenberg and Tatsien Li. The effect of boundary damping for the quasilinear wave equation. J. Differential Equations, 52(1):66-75, 1984. 
[20] Amaury Hayat. Exponential stability of general 1-D quasilinear systems with source terms for the $C^{1}$ norm under boundary conditions. Preprint, 2017.

[21] Amaury Hayat. On boundary stability of inhomogeneous $2 \times 21$-D hyperbolic systems for the $C^{1}$ norm. Preprint, 2017.

[22] Amaury Hayat and Peipei Shang. A quadratic Lyapunov function for Saint-Venant equations with arbitrary friction and space-varying slope. Preprint, 2017.

[23] Eberhard Hopf. The partial differential equation $u_{t}+u u_{x}=\mu u_{x x}$. Comm. Pure Appl. Math., 3:201-230, 1950 .

[24] Long Hu, Rafael Vazquez, Florent Di Meglio, and Miroslav Krstic. Boundary exponential stabilization of 1-d inhomogeneous quasilinear hyperbolic systems. arXiv preprint arXiv:1512.03539, 2015.

[25] Tosio Kato. The Cauchy problem for quasi-linear symmetric hyperbolic systems. Arch. Rational Mech. Anal., 58(3):181-205, 1975.

[26] Shigeo Kida. Asymptotic properties of Burgers turbulence. J. Fluid Mech., 93(2):337-377, 1979.

[27] Miroslav Krstic. On global stabilization of Burgers' equation by boundary control. Systems Control Lett., 37(3):123-141, 1999.

[28] Miroslav Krstic, Lionel Magnis, and Rafael Vazquez. Nonlinear stabilization of shock-like unstable equilibria in the viscous Burgers PDE. IEEE Trans. Automat. Control, 53(7):1678-1683, 2008.

[29] Peter D. Lax. Hyperbolic systems of conservation laws and the mathematical theory of shock waves. Society for Industrial and Applied Mathematics, Philadelphia, Pa., 1973. Conference $\overline{\text { Board }}$ of the Mathematical Sciences Regional Conference Series in Applied Mathematics, No. 11.

[30] Philippe LeFloch. Explicit formula for scalar nonlinear conservation laws with boundary condition. Math. Methods Appl. Sci., 10(3):265-287, 1988.

[31] Ta Tsien Li and Wen Ci Yu. Boundary value problems for quasilinear hyperbolic systems. Duke University Mathematics Series, V. Duke University, Mathematics Department, Durham, NC, 1985.

[32] Michael J. Lighthill. Viscosity effects in sound waves of finite amplitude. In Surveys in mechanics, pages 250-351 (2 plates). Cambridge, at the University Press, 1956.

[33] Andrew Majda. Compressible fluid flow and systems of conservation laws in several space

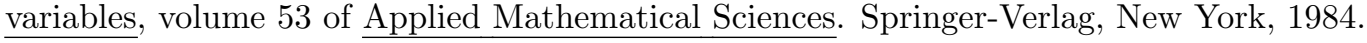

[34] Ol'ga Arsen'eva Oleı̆nik. Discontinuous solutions of non-linear differential equations. Uspehi Mat. Nauk (N.S.), 12(3(75)):3-73, 1957.

[35] Vincent Perrollaz. Asymptotic stabilization of entropy solutions to scalar conservation laws through a stationary feedback law. Ann. Inst. H. Poincaré Anal. Non Linéaire, 30(5):879-915, 2013.

[36] Vincent Perrollaz. Asymptotic stabilization of stationnary shock waves using a boundary feedback law. arXiv preprint arXiv:1801.06335, 2018.

[37] Sebastian Pfaff and Stefan Ulbrich. Optimal boundary control of nonlinear hyperbolic conservation laws with switched boundary data. SIAM J. Control Optim., 53(3):1250-1277, 2015.

[38] Jeffrey Rauch and Michael Taylor. Exponential decay of solutions to hyperbolic equations in bounded domains. Indiana Univ. Math. J., 24:79-86, 1974.

[39] David L. Russell. Controllability and stabilizability theory for linear partial differential equations: recent progress and open questions. SIAM Review, 20(4):639-739, 1978. 
[40] Jacques Simon. Compact sets in the space $L^{p}(0, T ; B)$. Ann. Mat. Pura Appl. (4), 146:65-96, 1987.

[41] Marshall Slemrod. Boundary feedback stabilization for a quasilinear wave equation. In Control theory for distributed parameter systems and applications (Vorau, 1982), volume 54 of Lect. Notes Control Inf. Sci., pages 221-237. Springer, Berlin, 1983.

[42] Andrey Smyshlyaev, Thomas Meurer, and Miroslav Krstic. Further results on stabilization of shock-like equilibria of the viscous Burgers PDE. IEEE Trans. Automat. Control, 55(8):19421946, 2010.

[43] Ying Tang, Christophe Prieur, and Antoine Girard. Singular perturbation approximation by means of a $H^{2}$ Lyapunov function for linear hyperbolic systems. Systems Control Lett., 88:2431, 2016.

[44] Tomomasa Tatsumi and Hiroshi Tokunaga. One-dimensional shock turbulence in a compressible fluid. J. Fluid Mech., 65:581-601, 1974. 